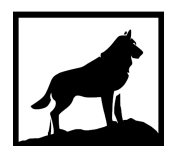

Michigan Technological

1 в 8 5 University
Michigan Technological University Digital Commons @ Michigan Tech

Dissertations, Master's Theses and Master's Reports

2016

HIGH RESOLUTION TIME-OF-ARRIVAL RANGING OF WIRELESS SENSOR NODES IN NON-HOMOGENOUS ENVIRONMENTS

Mohsen Jamalabdollahi

Michigan Technological University, mjamalab@mtu.edu

Copyright 2016 Mohsen Jamalabdollahi

Recommended Citation

Jamalabdollahi, Mohsen, "HIGH RESOLUTION TIME-OF-ARRIVAL RANGING OF WIRELESS SENSOR NODES IN NON-HOMOGENOUS ENVIRONMENTS", Open Access Dissertation, Michigan Technological University, 2016.

https://doi.org/10.37099/mtu.dc.etdr/268

Follow this and additional works at: https://digitalcommons.mtu.edu/etdr

Part of the Signal Processing Commons 


\title{
HIGH RESOLUTION TIME-OF-ARRIVAL RANGING OF WIRELESS SENSOR NODES IN NON-HOMOGENOUS ENVIRONMENTS
}

By

Mohsen Jamalabdollahi

\begin{abstract}
A DISSERTATION
Submitted in partial fulfillment of the requirements for the degree of DOCTOR OF PHILOSOPHY

In Electrical Engineering
\end{abstract}

MICHIGAN TECHNOLOGICAL UNIVERSITY

2016

Copyright 2016 Mohsen Jamalabdollahi 

This dissertation has been approved in partial fulfillment of the requirements for the Degree of DOCTOR OF PHILOSOPHY in Electrical Engineering.

Department of Electrical and Computer Engineering

Dissertation Advisor: Prof. Seyed (Reza) Zekavat

Committee Member: Prof. Daniel Fuhrmann

Committee Member: Dr. Zhaohui Wang

Committee Member: Dr. Alexander Labovsky

Department Chair: Prof. Daniel Fuhrmann 



\section{Dedication}

To my Beloved parents

who's the first priority were their children success. 



\section{Contents}

List of Figures . . . . . . . . . . . . . . . . . . Xiii

List of Tables . . . . . . . . . . . . . . . . . xvii

Preface . . . . . . . . . . . . . . . . . .

Abstract .............................

1 Introduction ....................... 1

1.1 Wireless Sensor Network Localization . . . . . . . . . . . . . 1

1.2 Applications of Localization in Non-Homogenous Media . . . . . . 4

1.2.1 Applications in Environmental Monitoring and Remote Sensing $\ldots \ldots \ldots \ldots \ldots \ldots \ldots \ldots \ldots \ldots \ldots \ldots \ldots \ldots$

1.2.2 Applications in Health Care . . . . . . . . . . . 5

1.3 Time-of-Arrival and Orthogonal Frequency Division Multiple Access 7

1.3.1 Time-of-Arrival Estimation $\ldots \ldots \ldots \ldots$

1.3.2 Orthogonal Frequency Division Multiple Access (OFDMA) 9

1.4 Chapters Contributions . . . . . . . . . . . . . . . 10 
2 Joint Neighbor Discovery and ToA Estimation in Wireless Sensor

Networks via OFDMA ................ 15

2.1 Introduction . . . . . . . . . . . . . . . 15

2.2 System Model . . . . . . . . . . . . . . . . . . . . . . . . 19

2.3 Joint ND and ToA Estimation .............. 21

2.3.1 Neighbor Discovery . . . . . . . . . . . . 22

2.3.2 ToA Estimation . . . . . . . . . . . . 27

2.3.3 Impact of the Number of Sub-Carriers . . . . . . . . . 31

2.4 Experimental Challenges . . . . . . . . . . . . . . . 34

2.4.1 Energy Efficiency and Scalability . . . . . . . . 34

2.4 .2 OFDMA Limitations . . . . . . . . . . . . 37

2.4.2.1 SFO and $\mathrm{CFO} \ldots \ldots \ldots$

2.4.2.2 PAPR .......................... 38

2.5 Simulation Results and Discussion . . . . . . . . . . . . . . 39

2.5.1 Simulation Parameters and Methods ......... 39

2.5.2 Neighbor Discovery Performance . . . . . . . . . 41

2.5.3 ToA Estimation Performance ........... 44

2.5.4 Energy Efficiency and Scalability Analysis ....... 50

2.6 Conclusions and Future Works . . . . . . . . . . . . 51

2.6.1 Conclusion ..................... 51

2.6.2 Future Works and Discussion .......... 52 


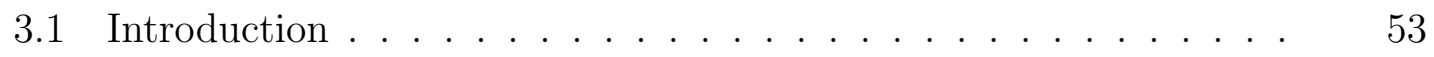

3.2 System Model and Problem Formulation . . . . . . . . . . 56

3.3 Proposed Technique . . . . . . . . . . . . . . . . . . . . . 60

3.3.1 Maximum rising level Detector . . . . . . . . . . . 60

3.3 .2 Waveform Design . . . . . . . . . . . . 66 66

3.3.2.1 Forming the objective function . . . . . . 66

3.3.2.2 Trust-region Algorithm . . . . . . . . . . . . 68

3.4 Simulation Results and Discussion . . . . . . . . . . . . 72

3.4.1 Simulation Parameters and Methods .......... 72

3.4.2 ToA Estimation Performance . . . . . . . . . . . 73

3.4.3 Complexity Analysis vs Performance . . . . . . . 75

3.4 .4 ToA CRLB . . . . . . . . . . . . . . . . . . 78

3.5 Conclusion . . . . . . . . . . . . . . . . . . . . 79

4 High Resolution ToA Estimation in Non-homogenous, Frequency

Dispersive Channels . . . . . . . . . . . . . . . . . . 81

4.1 System Model . . . . . . . . . . . . . . . . . . . . . . . 85

4.2 Proposed ToA Estimation Technique $\ldots \ldots \ldots \ldots$

4.2.1 Time Frame Detection Technique . . . . . . . . . . 89

4.2.2 High Resolution ToA Estimation . . . . . . . . . . . 91

4.3 Simulations Result and Discussions . . . . . . . . . . . 103 
4.3.1 Details of Simulated NH Media . . . . . . . . . . 103

4.3.2 Proposed Approximations Analysis . . . . . . . . 107

4.3.2.1 Overall delays linearity . . . . . . . . . . 107

4.3.2.2 Fine delay linearity . . . . . . . . . . . 109

4.3 .3 Performance Analysis . . . . . . . . . . . . . . . . . 110

4.4 Conclusion . . . . . . . . . . . . . . . . . . . . . . .

5 Range Measurements in Non-homogenous, Frequency Dispersive

Channels ....................... 117

5.1 System Model . . . . . . . . . . . . . . . . . . . . . . . 119

$5.2 \quad$ Proposed Technique. . . . . . . . . . . . . . . . . 122

5.3 Simulations Result and Discussions . . . . . . . . . . 124

5.4 Conclusion . . . . . . . . . . . . . . . . . . . 126

6 Conclusions and Future Works . . . . . . . . . . . . 129

6.1 Conclusions $\ldots \ldots \ldots \ldots \ldots$

6.2 Future Works . . . . . . . . . . . . . . . . . . . . . 131

References ........................ 135

A Proofs and Derivations .................. 151

A.1 Probabilities of Miss-Detection and False-Alarm . . . . . . 151

A.2 Probability of Error for ToA Estimation $\ldots \ldots \ldots \ldots$

A.3 First Order Derivative of WISL Objective Function . . . . . 160 
A.4 Second Order Derivative of WISL Objective Function . . . . . . . 163

A.5 Details of ToA CRLB Calculation . . . . . . . . . 164

A.6 Derivation of the Maximum Likelihood Estimator . . . . . . . . 166

A.7 Deriving Straight-Line Range . . . . . . . . . . . . . . . 168

B Publication Copyright Letter for Chapter 2 . . . . . . 171 



\section{List of Figures}

1.1 Absolute value of matched filter output. (a) propagated signal in free space (b) propagated signal in human body $\ldots . . . \ldots$

1.2 Transceiver block diagram for OFDM. . . . . . . . . . 9

2.1 Detection of sensor nodes signature in frequency domain. . . . . 25

2.2 Objective function for ToA estimation for different numbers of transmitted sub-carrier(s) . . . . . . . . . . . .

2.3 Probability of miss detection for neighbor discovery in MPFS channel using variable threshold. . . . . . . . . . . . . . 42

2.4 Probability of false alarm for neighbor discovery in MPFS channel using variable threshold. . . . . . . . . . . . . . .

2.5 Probability of miss detection for neighbor discovery in AWGN channel using fixed value threshold. . . . . . . . . . . . . . . . 43

2.6 Probability of false alarm for neighbor discovery in AWGN channel using fixed value threshold. . . . . . . . . . . . . . 44

2.7 Effect of allocated sub-carriers on the probability of miss detection. 45

2.8 Effect of allocated sub-carriers on the probability of false alarm. . 45 
2.9 Normalized mean square error (NMSE) of ToA estimation in AWGN channel considering different numbers of neighbor nodes. . . . . . 47

2.10 Normalized mean square error (NMSE) of ToA estimation in multipath channel considering different numbers of neighbor nodes. . . 47

2.11 Impact of increasing the number of transmitted sub-carriers in probability of error in ToA estimation. ........... 48

2.12 Impact of increasing the number of transmitted sub-carriers in NMSE of ToA estimator v.s. multi-band chirp signal . . . . . . . 48

2.13 Normalized mean square error (NMSE) of ToA estimation in multipath channel considering different numbers of neighbor nodes, v.s. multi-band chirp signal . . . . . . . . . . . . . . 49

2.14 Total current consumption value (mA), applying the TDMA based multi-band chirp and proposed method . . . . . . . . . 50 50

3.1 The block diagram of the proposed MRLD transceiver. . . . . . 63

3.2 Autocorrelation level corresponding to the over-sampled signal with $M=4$ and fine-resolution $k_{\tau_{0}}=3 \ldots \ldots \ldots$

3.3 Autocorrelation level of designed waveform, (a) $N=128$, (b) $N=256$ , (c) $N=512$, (d) $N=1024 \ldots \ldots \ldots$. . . . . . . . . 71

3.4 MSE of ToA exploiting different numbers of correlation paths $M$ vs SCAN with the same bandwidth $(1 \mathrm{MHz})$ in flat fading channel. . 
3.5 MSE of ToA exploiting different numbers of correlation paths $M$ vs SCAN with the same bandwidth $(1 \mathrm{MHz})$ in MPFS channels. . . .

3.6 Impact of proposed MRLD technique on coarse ToA estimation. .

3.7 Total required complex operations by the proposed technique. . .

3.8 Impact of increasing the number of parallel paths $M$ vs waveform length $N$, on the performance of proposed technique. . . . . .

4.1 Three different scenarios of received waveform, (a) close to the time fame start, (b) close to the end of time frame, (c) in the middle of

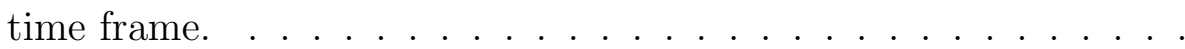

4.2 Applied NH media, (a) underwater-airborne, (b) four layers underground containing different water content in volume $\rho . \ldots \ldots$

4.3 Average time error imposed by linear approximation proposed in Re$\operatorname{mark} 1 \ldots \ldots \ldots \ldots \ldots \ldots \ldots \ldots \ldots$

4.4 Average time error imposed by linear approximation proposed in Re$\operatorname{mark} 2 \ldots \ldots \ldots \ldots \ldots \ldots \ldots \ldots \ldots \ldots$

4.5 Impact of the number of allocated subcarriers and transmitted signal length on the estimated ToA average error in human body, (a) $N=$ 512, (b) $N=1024$, (c) $N=2048$, and (d) $N=4096 \ldots \ldots \ldots$ 
4.6 Impact of the number of allocated subcarriers and transmitted signal length on the estimated ToA average error in airborne to underwater channel, (a) $N=512$, (b) $N=1024$, (c) $N=2048$, and (d) $N=$ 4096............................. 113

4.7 Impact of the number of allocated subcarriers and transmitted signal length on the estimated ToA average error in underground channel, (a) $N=512$, (b) $N=1024$, (c) $N=2048$, and (d) $N=4096$.

$5.13 \mathrm{D}$ model of signal propagation in $\mathrm{NH}$ media. . . . . . . . . 121

5.2 Impact of the number of applied measurements $Q$ and ToA measurements error on the estimated range, (a) underwater-airborne channel, (b) underground channel. . . . . . . . . . . . . . . . . 126

5.3 Impact of the number of applied measurements $Q$ and DoA measurements error on the estimated range, (a) underwater-airborne channel, (b) underground channel. . . . . . . . . . . . . . . . 127 


\section{List of Tables}

2.1 Active transmission $\left(T_{x}\right)$ and reception $\left(T_{r}\right)$ and the total number of real-value instructions at target (Tar.) and neighbor (Nei.) nodes for ND and ToA estimation of the proposed method (Pro.) and multi-band chirp exploiting the time-slot based approaches $\ldots \ldots \ldots \ldots \ldots \ldots \ldots$

2.2 Simulation parameters and applied values $\ldots \ldots \ldots$

3.1 Simulation parameters and applied values $\ldots \ldots \ldots$

4.1 Simulation parameters and applied values . . . . . . . 104

4.2 Example of applied relative permittivities vs number of allocated sub-carriers (bandwidth) for $N=1024 \ldots \ldots \ldots$

4.3 Relative permittivities at different applied carrier frequen$\operatorname{cies}(\mathrm{GHz}) . \ldots \ldots \ldots \ldots \ldots \ldots$ 



\section{Preface}

The goal of this dissertation is to address ranging of wireless senor nodes in nonhomogenous media. Materials in chapter 2 is published, and materials in chapters 3, 4 and 5 are submitted for publication.

Publications list as follow:

1. Mohsen Jamalabdollahi, Seyed Reza Zekavat, Joint Neighbor Discovery and Time of Arrival Estimation in Wireless Sensor Networks via OFDMA, DOI 10.1109/JSEN.2015.2449079, IEEE Sensors Journal, June 2015.

This paper is original work of M. Jamalabdollahi as researcher, writer and corresponding author. Professor S. R. Zekavat is contributed as research advisor. He helped with preparation of this manuscript by several meetings and reviewing and revising the manuscript. The copy right permission is granted from IEEE and is available in Appendix B.

2. Mohsen Jamalabdollahi, Seyed Reza Zekavat, High Resolution ToA Estimation via Optimal Waveform Design, Submitted, Second round of review, IEEE Transaction on Communications, July 2016.

This paper is original work of M. Jamalabdollahi as researcher, writer and 
corresponding author. Professor S. R. Zekavat is contributed as research advisor. He helped with preparation of this manuscript by several meetings and reviewing and revising the manuscript.

3. Mohsen Jamalabdollahi, Seyed Reza Zekavat, High Resolution ToA Estimation in Non-homogenous, Time and Frequency Dispersive Channels, Submitted, IEEE Transaction on Signal Processing, March 2016.

This paper is original work of M. Jamalabdollahi as researcher, writer and corresponding author. Professor S. R. Zekavat is contributed as research advisor. He helped with preparation of this manuscript by several meetings and reviewing and revising the manuscript.

4. Mohsen Jamalabdollahi, Seyed Reza Zekavat, Range Measurements in Nonhomogenous, Time and Frequency Dispersive Channels via Time and Direction of Arrival Merger, Accepted, IEEE Transaction on Geoscience and Remote Sensing, July 2016.

This paper is original work of M. Jamalabdollahi as researcher, writer and corresponding author. Professor S. R. Zekavat is contributed as research advisor. He helped with preparation of this manuscript by several meetings and reviewing and revising the manuscript. 


\section{Abstract}

Wireless Sensor Networks (WSN) have emerging applications in homogeneous environments such as free space. In addition, WSNs are finding new applications in non-homogeneous (NH) media. All referred applications entail location information of measured data or observed event. Localization in WSNs is considered as the leading remedy, which refers to the procedure of obtaining the sensor nodes relative location utilizing range measurements. Localization via Time-of-Arrival (ToA) estimation has received considerable attention because of high precision and low complexity implementation, however, the traditional techniques are not feasible in $\mathrm{NH}$ media due to frequency dispersion of transmitted ranging waveform.

In this work, a novel and effective ToA-based ranging technique for localization in NH media consisting of frequency dispersive sub-media is proposed. First challenges of ToA estimation in NH media regarding frequency dispersion is investigated. Here, a novel technique which improves ToA estimation resolution at fixed bandwidth via maximum rising level detector (MRLD) technique is discussed. The MRLD receiver utilizes oversampling and multiple correlation paths to evaluate with high resolution the path corresponding to the maximum rising level of matched filters output. 
In order to achieve higher resolution, a novel and effective ToA estimation is introduced that incorporates orthogonal frequency division multiple access (OFDMA) subcarriers. In the proposed technique, pre-allocated orthogonal subcarriers are utilized to construct a ranging waveform which enables high performance ToA estimation in dispersive NH media in frequency domain. Here, we show that each frequency component of propagated waveform is received with different time delay and phase which dramatically increases the number of unknowns in the received signal system model. Then, we propose a novel idea based on frequency domain analysis of the transmitted OFDMA subcarriers to reduce the number of unknowns exploiting feasible approximations.

Finally, the proposed ToA technique is applied multiple times at different carrier frequencies to create a system of linear equations which can be solved to compute the available sub-mediums thickness and range. Simulation results prove that the proposed technique offers high resolution range measurements given simulated ToA estimation error at different signal to noise ratio regimes in $\mathrm{NH}$ media. 


\section{Chapter 1}

\section{Introduction}

\subsection{Wireless Sensor Network Localization}

Wireless Sensor Networks (WSN) have emerging applications in homogeneous environments such as free space [1] [2] [3]. Examples are environmental monitoring [4] [5, 6, 7, 8, 9], search and rescue [10][11][12], health [13][14][15] and body area networking [16][17][18], road traffic monitoring [19] [20] [21], and pollution sensing [22, 23]. In addition, WSNs are finding new applications in non-homogeneous (NH) channels. Examples are endoscopy capsule localization [24, 25] cancer detection [26, 27, 28], drug-delivery [29] [30, 31, 32, 33] [34], underwater monitoring and surveillance [35] and underground and mine safety and monitoring [36, 37]. Here, the term 
non-homogenous refers to a medium composed of diverse dispersive mediums such as water, ice, soil, human body organs, ionosphere layers, and free space. All referred applications entail location information of measured data or observed event. Localization in WSNs is considered as the leading remedy, which refers to the procedure of obtaining the sensor nodes location utilizing range measurements (range based) [27, 38], [39, 40, 41, 42, 43, 44, 45, 46, 47, 48, 49, 50, 51, 52, 53, 54, 55, 56, 57, 58].

Range-based approaches offer higher localization accuracy [38, 39, 40], 44, 42, 43, 44, and require minimal network connectivity, which compared to range free techniques makes them attractive for many applications such as drug delivery, and cancer detection. Range based localization techniques for WSN include received signal strength (RSS) [39, 41, 42, 43] [44] [59], time-of-arrival (ToA) [38] [45, 46, 47, 48] 49], time-difference-of-arrival (TDOA) [27, 50, 51, 52] [53], and direction-of-arriving (DOA) [54][55, [56, 57, 58]. In all offered ranging methods, ToA estimation has received considerable attention because of high precision and low complexity implementation [40], 38].

Although, a few works [60] 61, 62, 63] 64, have discussed ranging and localization in dispersive mediums such as underwater or seismic, and body [25, 65, however, no work can be observed that investigates ranging algorithms within non-homogenous mediums. To the best of author knowledge, high resolution ToA estimation exploiting wideband microwave signals in NH media or single medium in frequency 
dispersion band is an open problem. Many researchers propose acoustics communication for ToA estimation in underwater or underground media [66]. Although acoustics communication offers high resolution for underwater ToA estimation, it cannot be applied to airborne-underwater channels due to strong reflections and attenuation in water/air boundary [67]. A few works [61] 63], 64, 68], 69] propose ToA estimation in dispersive medium for specific scenarios which cannot be extended to NH media. In [68] and 69], time delays estimation of buried target echoes for ground penetration radar (GPR) is addressed, meanwhile, the received echoes do not represent frequency dispersion assuming low conductivity and small layer thickness of sub-media. Although, these assumptions can be feasible for dry media, however, when the water content of media exceeds $10 \%$ by weight, the frequency dispersion must be considered due to the dielectric relaxation of water [70]. In [61], the transmitter location is estimated by combining the measured ToA data with the knowledge about shape and position of the medium which is not a feasible assumption in NH media. Authors in [63], propose sensor node localization via ToA measurements meanwhile the procedure of ToA estimation in soil as frequency dispersive medium is not discussed. In 64, ToA estimation of short range seismic signals in dispersive environments is addressed, however the system model does not represent the frequency dispersion due to low bandwidth of applied seismic signals. Thus, In this thesis, the following problem are defined and address: 


\subsection{Applications of Localization in Non- Homogenous Media}

\subsubsection{Applications in Environmental Monitoring and Re- mote Sensing}

Range measurements in NH media opens a new research area in localization and scanning technologies such as Light/Laser Detection and Ranging (LiDAR) and GPR in frequency dispersive media where no resolution restriction is needed to prevent frequency dispersion of ultra wide band waveforms. As the first example, underwater scanning scenario via airborne sensors is considered. Here, the airborneunderwater channel is a NH media and requires the proposed technique for ToA and straight line range measurements for localization. Although acoustics communication offers high resolution for underwater ToA estimation, it cannot be applied to airborne-underwater channels due to strong reflections and attenuation in water-air boundary. Underwater target/sensor detection and or localization via airborne radar is a special case of this type of media with verity of applications such as military or environmental monitoring.

Multilayer underground channel with different water content is another example of 
$\mathrm{NH}$ media. Here, a buried sensor node in the multilayer ground is considered where the thickness of available layers and the straight line range between sensor node and receiver on the ground surface is achievable via the proposed technique. Although, GPR can propose the thickness of multilayer underground channels, it cannot be applied to straight line range measurements. Mine tunnel safety via senor nodes is an important application of this scenario where each sensor node needs underground range measurements for localization.

\subsubsection{Applications in Health Care}

Human body is an NH medium which requires straight-line range measurement for localization. Localization of endoscopy capsules or cancer detection and/or drug delivery exploiting wireless nano-sensor networks are applications of 3D localization of a sensor node within $\mathrm{NH}$ channels of human body. As the first application, one can propose approaches on localization and tracking of endoscopy capsules by applying the proposed straight line range measurements technique. The expected improvements in endoscopy capsule localization and tracking can be subsequently exploited to develop a simple platform for clinical evaluation of the human digestive system that are unreachable or extremely invasive when viewed via cable endoscopy. 
In addition, there are other futuristic applications for WSNs in health care. Recently, with advances in Micro-Electrical-Mechanical Systems (MEMS), the idea of nano-sensors has been developed. Nano-sensors have diverse applications, including applications in the human body such as cancer detection and drug delivery. Existing cancer detection methods, such as magnetic resonance imaging (MRI), computed tomography scans, and mammography, are not ideal for whole-body cancer screening due to their high cost and/or low sensitivity. Moreover, these methods possess low accuracy, particularly at the early stages of cancer tumor developmentexactly the time when treatment could be most effective. To tackle the drawbacks of existing methods, the idea of a cancer detection approach that incorporates magnetic nano-particles (MNPs) has gained attention. However, MNPs are not active devices and cannot use networking features (e.g., cooperative sensing and data sharing) that greatly improve the efficiency and performance of cancer cell detection. Nano-sensor networks are formed by a large number of nano-sensors with processing, communication, and networking capabilities. The processing, communication and networking capabilities make each nano-sensor a powerful particle. Meanwhile, localization of these particles intensifies their capabilities on cancer detection and/pr drug delivery which cannot be achieved without range measurement techniques in NH media. 


\subsection{Time-of-Arrival and Orthogonal Frequency Division Multiple Access}

\subsubsection{Time-of-Arrival Estimation}

Time-of-Arrival (ToA) is the most popular and precise range measurement. Rather than localization, ToA estimation has other applications in radar systems or wireless cellular networks. ToA estimation techniques are divided into two different categories, the traditional matched filter-based techniques, and the super resolution techniques. The former incorporates a pre-designed waveform with autocorrelation properties close to the delta function at the output of matched filter. In later, ToA is calculated via maximizing the pseudo-spectrum of the corresponding signal subspace achievable via decomposition of the matched filter output in frequency domain [71, 72]. Examples of super resolution techniques are independent component analysis (ICA) [73], maximum likelihood (ML) [74], multiple signal classification (MUSIC) [75]-77] and estimation of signal parameter via rotational invariance technique (ESPRIT) [78]. However, the resolution of all proposed techniques depends on the transmitted waveform bandwidth [73]. Figure 1.1(a) depicts the output of matched 


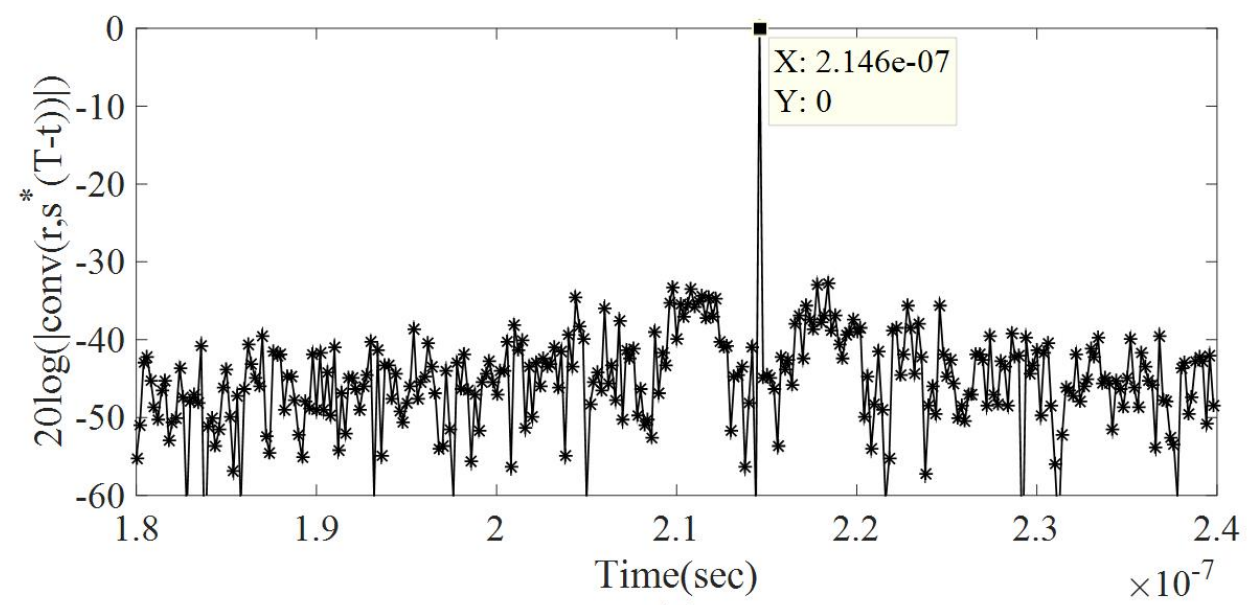

(a)

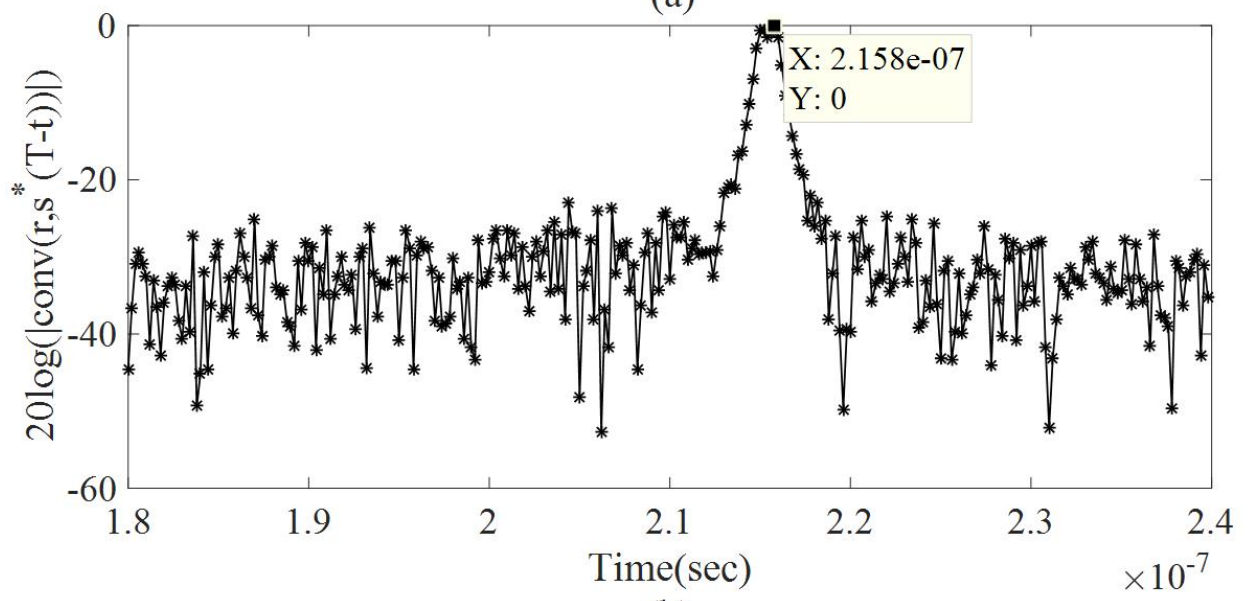

(b)

Figure 1.1: Absolute value of matched filter output. (a) propagated signal in free space (b) propagated signal in human body

filter output regarding transmission of Golomb signal in multipath wireless channels. As shown, the estimated ToA which corresponds to the time index of filter output maximum is equal to the actual delay. However, for NH media different scenario is observed exploiting traditional matched filter technique as shown in Figure 1.1(b). Here, the transmitted signal is propagated over human body. As shown, the received signal is dispersed which causes sidelobes which moves the maximum value into other time indexes. Therefore, proposing a new ToA estimation approach 


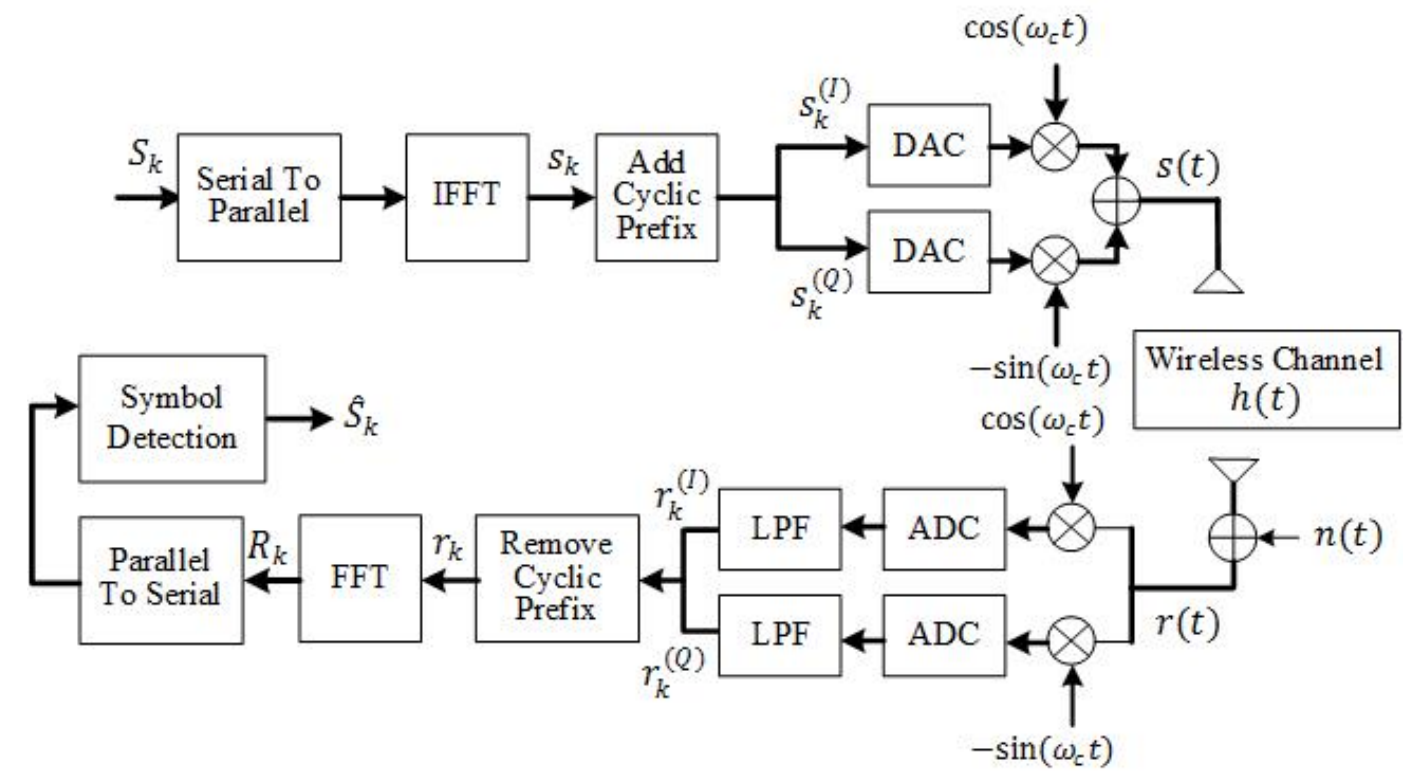

Figure 1.2: Transceiver block diagram for OFDM.

consistent with NH media including frequency dispersive sub-media is necessary.

\subsubsection{Orthogonal Frequency Division Multiple Access (OFDMA)}

The idea of Orthogonal Frequency Division Multiplexing (OFDM) backs to 70's [79, 80, 81, 82, 83. OFDM enjoys the very fact that circular convolution in discrete time domain can be represented by multiplication in frequency domain. This simple idea is extensively widespread in digital communication system such as WiFi [84], WiMax [85], LTE [86], and etc. Figure 1.2 represents the block diagram of OFDM transmitter and receiver. Here each data symbol is transmitted with an orthogonal 
subcarrier (OFDMA sub-carrier) which has less bandwidth than the original signal. Therefore, frequency selective channels perform as frequency flat fading for each transmitted sub-carrier which removes the inter symbol interference in the received signal in frequency domain. The term Orthogonal Frequency Division Multiple Access (OFDMA) is special case of OFDM system where a part of available subcarrier are allocated into each user which enables multiple access through the wireless channel. For more information on $\operatorname{OFDM}(\mathrm{A})$ we refer reader to [87].

\subsection{Chapters Contributions}

In this section the contributions of each chapter are discussed.

\section{Joint Neighbor Discovery and Time of Arrival Estimation in Wire-} less Sensor Networks via OFDMA: Chapter 2 introduces joint neighbor discovery (ND) and coarse time-of-arrival (ToA) estimation in wireless sensor networks (WSN) via orthogonal frequency division multiple access (OFDMA). In the proposed technique, each sensor node exploits at least one orthogonal sub-carrier as its allocated signature, to respond the ND and ToA estimation requests transmitted by target nodes. The target node utilizes the orthogonality across sub-carriers to detect the transmitted signatures and their corresponding delays. This technique is energy efficient as it avoids multiple 
transmissions and receptions inherent in traditional neighbor discovery protocols and ToA estimation techniques in WSN. Moreover, in this technique, network initiation process does not require channel information or time synchronization across sensor nodes. The performance of the proposed method is studied by evaluating the probabilities of false alarm and miss detection of the neighbor discovery. In addition, ToA estimation error is calculated theoretically and via simulations. Moreover, the impact of available bandwidth on the performance and energy efficiency of ND and ToA estimation are investigated. Simulation results confirm the energy efficiency and the feasibility of the proposed method even at low signal to noise ratio (SNR) regimes and in multi-path and frequency selective (MPFS) channels.

\section{High Resolution ToA Estimation via Optimal Waveform Design:} Chapter 3 introduces a novel method to improve the Time of Arrival (ToA) estimation resolution for a fixed available bandwidth in the presence of unknown multipath frequency selective (MPFS) channels. Here, the maximum rising level detector (MRLD) technique is proposed which utilizes oversampling and multiple correlation paths to evaluate with high resolution the path corresponding to the maximum rising level of matched filters output. However, employing such technique demands for transmission of waveform that creates a very high rising level at autocorrelation center. This chapter proposes an efficient technique to design proper waveforms (very high rising level 
at autocorrelation center) via minimization of weighted integrated sidelobe level (WISL), exploiting the trust-region algorithm. The performance of the proposed technique is evaluated via simulations of ToA mean square error (MSE), and compared to the state-of-the-art approaches considering the same bandwidth, and Cramer-Rao lower bound (CRLB) as benchmark. Simulations confirm that ToA resolution is improved as the number of correlation paths increases and verify the feasibility of the proposed technique compared to the available approaches for MPFS channels.

\section{High Resolution ToA Estimation in Non-homogenous, Frequency}

Dispersive Channels: Chapter 4 introduces a novel and effective time-ofarrival (ToA) estimation in Non-homogenous (NH) media consisting of frequency dispersive sub-media via orthogonal frequency division multiple access (OFDMA) subcarriers. In the proposed technique, pre-allocated orthogonal subcarriers are utilized to construct a ranging waveform which enables high performance ToA estimation in dispersive $\mathrm{NH}$ media in frequency domain. First, time and frequency domain system models for propagation of arbitrary waveform in frequency dispersive media is proposed. Here, we show that each frequency component of propagated waveform is received with different time delay and phase which dramatically increases the number of unknowns in the received signal system model. Then, we propose a novel idea based on frequency domain analysis of the transmitted OFDMA subcarriers which reduces 
the number of unknowns exploiting feasible approximations. The exploited approximations are discussed via theoretical evaluations and simulations for diverse scenarios to prove the feasibility of the proposed technique. Simulation results prove that the proposed technique offers high resolution ToA estimation in NH media with diverse required resolution and/or propagation distance such as human body or airborne-underwater channels.

\section{Range Measurements in Non-homogenous, Frequency Dispersive}

Channels: Chapter 5 introduces a novel and effective range measurements in Non-homogenous (NH) media consisting of frequency dispersive sub-media via time-of-arrival (ToA). The proposed ToA technique is exploited for multiple ToA measurements at different carrier frequencies. The proposed measurements leads to a system of linear equations which can be solved to reveal the available sub-mediums thickness and range. Simulation results prove that the proposed technique offers high resolution range measurements given simulated ToA estimation error at different signal to noise ratio regimes in $\mathrm{NH}$ media.

The proposed problems have significant theoretical and technological impacts. The proposed thesis defines new measures unique to NH environments critical to many futuristic applications in harsh environments that include but not limited to health 
care, underwater, and seismology sensors, as well as sensors used for explore and extraction in oil and mine industries. The criteria for optimizing ranging and localization distribution to enable energy efficiency are implementable in many cooperative scenarios in WSN such as routing, tracking and etc. 


\section{Chapter 2}

\section{Joint Neighbor Discovery and ToA}

\section{Estimation in Wireless Sensor}

Networks via OFDMA 1

\subsection{Introduction}

Time-of-arrival (ToA) estimation has received considerable attention because of high precision and low complexity [38],40]. Although, ToA based ranging methods are precise and seem proper for sensor networks, clock synchronization across sensor

${ }^{1}$ The material contained in this chapter was previously published in IEEE Sensor Journal, vol. 15, pp. 5821-5833, 2015 
nodes remain a significant issue for these techniques in ToA based ranging approaches. To mitigate this problem, TDoA method which subtracts the pairwise ToA measurements to eliminate the clock offset have been proposed [46], however, this subtraction increases the measurement noise by $3 \mathrm{~dB}$ [38]. In wireless local positioning system (WLPS) [88, the round trip scenario for ToA measurements is proposed which mitigates the clock synchronization problem, however it is not efficient for dense sensor networks due to the high probability of collision of signals submitted by nodes. In [89, [90] authors utilize time-slot based approaches to avoid signal collision. Although these methods are feasible at dense networks, they are not energy efficient due to the high required numbers of signal transmissions and receptions. Note that, up to $80 \%$ of energy in wireless sensor nodes is consumed by the radio communication process [91].

Time-of-arrival (ToA) estimation techniques are divided into two different categories, the traditional techniques that are based on the matched filter output, and the super resolution techniques. The former incorporates a pre-designed waveform with autocorrelation properties close to the delta function at the output of matched filter. Although correlation based techniques propose excellent performance in the presence of unknown multipath frequency selective (MPFS) channels, however, their proposed ToA resolution is limited by transmitted waveform bandwidth. In later, ToA is calculated via maximizing the pseudo-spectrum of the corresponding signal sub-space achievable via decomposition of the matched filter output in frequency 
domain [71, 72]. Examples of super resolution techniques are independent component analysis (ICA) [92], maximum likelihood (ML) [74], multiple signal classification (MUSIC) [75]-77] and estimation of signal parameter via rotational invariance technique (ESPRIT) [78]. However, the frequency domain techniques only improve the ToA resolution in flat fading channels or in the presence of multiple resolvable paths which are not feasible assumptions in many ToA applications. Moreover, these works investigate the single user while in WSN, it is more energy efficient to apply a procedure for all neighbors simultaneously and to avoid multiple signal transmissions for ranging 90 .

Applying the aforementioned ToA estimation techniques require the information of the number of available sensor nodes and their ID within the radio range of target node. The process of discovering the available sensors within the radio range of a target nodes is called neighbor discovery (ND). Neighbor discovery in WSN has been addressed in many works [93] 94, 95, 96, 97, 98, 99]. Some of ND approaches [93] 94] [95], propose a protocol based technique, which needs multiple number of radio transmissions and receptions that can not be considered energy efficient [91]. Other approaches apply a signal processing technique to reduce the energy consumption [96]-98]. Here, authors apply direct sequence code division multiple access (DS-CDMA) and compressed sensing approaches respectively, to address neighbor discovery over flat channels, however the case of multi-path and frequency selective (MPFS) channel still remains open. Luo and et al. [99] have considered the Rayleigh 
fading case, however, the system model does not include the signal specification and how they tackle multiuser interference. Moreover, none of the proposed techniques integrates the ND and ranging processes.

Here, we propose a novel energy efficient neighbor discovery and ToA estimation achievable via orthogonal frequency division multiple access (OFDMA). This maintains energy efficiency because the proposed technique requires only one transmission and one reception for ND and ToA estimation procedures per sensor node. The orthogonality of transmitted signatures by sensor nodes, enables the receiver to discover available neighbors and the propagation delay of each detected signature over MPFS channels. Moreover, the orthogonality of transmitted signatures addresses the problem of received signal collision for ToA estimation. The idea of exploiting $\operatorname{OFDM}(\mathrm{A})$ is studied by many works [100, $, 82,101,102]$ and [83], however, none of these works have addressed ND or ToA estimation. In addition to the experimental challenges of OFDMA, the performance of the proposed method is investigated by evaluating the probabilities of miss detection and false alarm for neighbor discovery theoretically and via simulations over AWGN and MPFS channels. Moreover, the probability of correct coarse ToA estimation is investigated theoretically and via simulations. Furthermore, the impacts of allocated bandwidth to each sensor node on the performance of ToA estimation and ND are investigated. The normalized mean square error (NMSE) of ToA has been simulated and compared to multi-band chirp signal proposed in [78]. Finally, the energy efficiency and the scalability of proposed 
method is studied by simulation of consumed energy for the proposed technique and the multi-band chirp signal [78] employing the time-slot based approaches [89], [90] at MAC layer, and the probability of sub-carrier collision respectively.

The rest of this chapter is organized as follow. Section 2.2 introduces the system model. The proposed algorithm for joint neighbor discovery and ToA estimation is presented in Section 2.3. Section 2.4 discusses the experimental challenges of the proposed technique. Section 2.5 represents simulation results and discussions and finally Section 2.6 concludes this chapter.

\subsection{System Model}

Consider a WSN with $M_{T}$ sensor nodes in which the target node initiates ND and ToA estimation process by transmitting a request signal through the network. Applying round-trip ranging, each neighbor node responds to the received request signal via its allocated signature defined based on OFDMA transmission, without any delay. Here, the received baseband signal by the target node over an $L$-path channel corresponds to:

$$
r(t)=\sum_{m=1}^{M_{T}} \gamma^{(m)} \sum_{l=0}^{L-1} h_{l}^{(m)} s^{(m)}\left(t-\tau_{l}^{(m)}\right)+v(t),
$$


where, $M_{T}, h_{l}^{(m)}$ and $\tau_{l}^{(m)}, s^{(m)}(t)$ and $v(t)$ represent total number of sensor nodes, the gain and delay of the $l^{\text {th }}$ tap of channel impulse response between the $m^{\text {th }}$ sensor node and target node, transmitted signature by the $m^{\text {th }}$ sensor node and additive white zero mean Gaussian noise, respectively. Moreover, $\gamma^{(m)}$, is the active sensor coefficient which equals to 1 when the $m^{\text {th }}$ sensor node is the neighbor of the target node ( $i^{\text {th }}$ sensor node) and 0 , otherwise. Applying (2.1) to the analog to digital converter with the sampling rate of $f_{s}=1 / T_{s}$ where $T_{s}$ is considered as sample interval of baseband signal leads to:

$$
\begin{aligned}
r(k)=\sum_{m=1}^{M_{T}} \gamma^{(m)} \sum_{l=0}^{L-1} h_{l}^{(m)} s^{(m)}\left(k T_{s}-\tau_{l}^{(m)}\right) & \\
& \quad+v\left(k T_{s}\right), \text { for } 0 \leq k \leq L_{s}-1,
\end{aligned}
$$

where $L_{s}$ denotes the length of the received signal, $M_{T}, L, h_{l}^{(m)}, \tau_{l}^{(m)}$ and $\gamma^{(m)}$ are defined in 2.1$)$ and $s^{(m)}\left(k T_{s}\right)$ and $v\left(k T_{s}\right)$ represent the $k^{t h}$ sample of the transmitted signature by the $m^{\text {th }}$ sensor node and additive noise, respectively. Given $R$ and $c$ as the maximum possible radio range of sensor nodes and the universal physical constant speed of light, respectively, the target node samples the channel for the duration of $T=2 R / c$ to receive response from all available neighbor nodes. Thus, the length of the received signal by target node $\left(L_{s}\right)$ is:

$$
L_{s}=T / T_{s}+T_{\text {Proc }} / T_{s}+L_{\text {symb }}
$$


where $T_{s}$ and $L_{s}$ are defined in (2.1) and $L_{s y m b}$ and $T_{\text {Proc }}$ denote the length of sensors signature for all sensor nodes and the required time for processing of transmitted request and responding it in neighboring nodes, respectively. The target node aims to estimate $\gamma^{(m)}$ and $\tau_{0}^{(m)}$ for $m \in M$ where $M\left(M \leq M_{T}\right)$ denotes the number of all available neighbor nodes among $M_{T}$ sensor nodes within the network. In the following section, the structure of each sensor's response and the algorithm for

estimation of $\gamma^{(m)}$ and $\tau_{0}^{(m)}$ based on the orthogonality of transmitted signature $\left(s^{(m)}(t)\right)$ is discussed.

\subsection{Joint ND and ToA Estimation}

The orthogonality of pre-allocated signature of each sensor node is the key to the proposed ND and ToA estimation methods. In OFDMA, orthogonal baseband subcarriers are dynamically allocated to each user for data transmission. These subcarriers are considered as the unique signature of each user. Therefore, the $m^{\text {th }}$ sensor node's signature $\left(s^{(m)}(k)\right)$ is represented by:

$$
s^{(m)}(k)=\sum_{p \in \mathcal{N}_{m}} e^{j 2 \pi p \Delta f k T_{s}} \text { for } 1 \leq k \leq N,
$$

where $\mathcal{N}_{m}$ denotes the set of $N_{s}$ sub-carrier indexes allocated to the $m^{\text {th }}$ sensor node's signature with length $N$ and $\Delta f$ represents the sub-carrier spacing, and $T_{s}$ 
is defined in (2.1). To maintain orthogonality across the sensor node's signature with $N$ samples and sample duration $T_{s}$, the sub-carrier spacing must satisfy $\Delta f=$ $1 /\left(N T_{s}\right)$. Applying $\Delta f=1 /\left(N T_{s}\right)$ into 2.4 , leads to $s^{(m)}(k)=e^{j 2 \pi m k / N}$ for $N_{s}=1$. Here, for simplicity, one sub-carrier is considered for each senor node's signature; however, the same procedure can be applied in the case of $N_{s}>1$. Considering $\mathbf{s}^{(m)}=\left[s^{(m)}(1), s^{(m)}(2), \ldots, s^{(m)}(N)\right]^{T}$, the orthogonality across sensor node's signature implies that:

$$
\left(\mathbf{s}^{(m)}\right)^{H} \mathbf{s}^{(n)}= \begin{cases}N & \text { for } n=m, \\ 0 & \text { for } n \neq m,\end{cases}
$$

where $(.)^{H}$ denotes transpose-conjugate, and $N$ is the length of sensor's signature. Considering the allocated signature defined in 2.4 , the following subsections introduce our proposed methods for ND and ToA estimation in WSN.

\subsubsection{Neighbor Discovery}

The neighbor discovery process starts with the transmission of an initiation request from the target node through the network. Without loss of generality, consider the $m^{\text {th }}$ sensor node as the target node which transmits the initiation request signal. Applying the round-trip based scenario for joint neighbor discovery and ToA 
estimation, it is desired that all neighbor nodes receive the initiation request and respond to it by transmitting their signature with no delay. Considering (2.1) as the corresponding system model for $L_{s}$ samples of received signal, the target node multiplies the received signal by $W$ to detect the transmitted neighbors signature, where $\mathbf{W}=\left[\mathbf{w}_{1}, \mathbf{w}_{2}, \ldots, \mathbf{w}_{M_{T}}\right]$ is an $M_{T} \times L_{s}$ DFT matrix such that:

$$
w_{n}(k)=e^{-j 2 \pi n \Delta f k T_{s}} \text { for } 0 \leq k \leq L_{s}
$$

represents the $k^{t h}$ entry of column vector $\mathbf{w}_{n}$ and $\Delta f$ and $T_{s}$ are the sub-carrier spacing and sampling time used in (2.4). Applying $\Delta f=1 /\left(N T_{s}\right)$ into (2.6) leads to:

$$
\mathbf{w}_{n}=\left[1, e^{-j 2 \pi n / N}, e^{-j 2 \pi 2 n / N}, \ldots, e^{-j 2 \pi n L_{s} / N}\right]^{T},
$$

Here, 2.7) implies that, the $n^{\text {th }}$ row of DFT matrix $(\mathbf{W})$ is the conjugate of $L_{s^{-}}$ sample expansion of the $n^{\text {th }}$ sensor node's signature where $L_{s}$ is defined in 2.3 . In other words, the $n^{\text {th }}$ row of DFT matrix contains the matched filter of the $n^{\text {th }}$ sensor node's signature, followed by a long cyclic prefix with length $L_{s}-N$. This maintains orthogonality across all $L_{s}$ samples of the received signal and the $n^{\text {th }}$ row of $\mathbf{W}$ regardless of which sample of the received signal corresponds to the first sample of $n^{\text {th }}$ sensor's signature. Considering $\mathbf{y}=\mathbf{W r}$, the $n^{\text {th }}$ entry of vector $\mathbf{y}$ is 
represented by:

$$
y_{n}=\mathbf{w}_{n}^{T} \mathbf{r}=\sum_{k=0}^{L_{s}-1} \sum_{m=1}^{M_{T}} \gamma^{(m)} \sum_{l=0}^{L-1} h_{l}^{(m)} w_{n}(k) s^{(m)}\left(k T_{s}-\tau_{l}^{(m)}\right)+\sum_{k=0}^{L_{s}-1} w_{n}(k) v\left(k T_{s}\right),
$$

where $M_{T}, L, \gamma^{(m)}, h_{l}^{(m)}, \tau_{l}^{(m)}, s^{(m)}(t)$ and $v(t)$ are defined in 2.1 and $L_{s}$ and $w_{n}(k)$ are defined in $(2.3)$ and $(2.6)$, respectively. The orthogonality of sub-carriers corresponds to:

$$
\sum_{k=0}^{L_{s}-1} w_{n}(k) s^{(m)}\left(k T_{s}-\tau_{l}^{(m)}\right)=\left\{\begin{array}{l}
N e^{-j 2 \pi \Delta f \tau_{l}^{(m)}} n=m \\
0 \quad \text { otherwise }
\end{array}\right.
$$

where $N$ denotes the length of transmitted signature, $h_{l}^{(m)}, \tau_{l}^{(m)}$ and $v(t)$ are defined in (2.1) and $L_{s}$ and $w_{n}(k)$ are defined in $(2.3)$ and (2.6), respectively. Applying (2.9) into 2.8, the $n^{\text {th }}$ entry of $\mathbf{y}$ is represented by:

$$
y_{n}=\mathbf{w}_{n}^{T} \mathbf{r}= \begin{cases}N \sum_{l=0}^{L-1} h_{l}^{(n)} e^{-j 2 \pi \Delta f \tau_{l}^{(n)}}+\sum_{k=0}^{L_{s}-1} w_{n}(k) v\left(k T_{s}\right) & n=m, \\ \sum_{k=0}^{L_{s}-1} w_{n}(k) v\left(k T_{s}\right) & n \neq m,\end{cases}
$$

where $N$ denotes the length of transmitted signature, $L, h_{l}^{(m)}, \tau_{l}^{(m)}$ and $v(t)$ are defined in 2.1) and $L_{s}$ and $w_{n}(k)$ are defined in (2.3) and (2.6), respectively. The target node, calculates the absolute value of all $M_{T}$ entries of $\mathbf{y}$ and compares them with a threshold to discover the transmitted signatures. Figure 2.1 depicts 


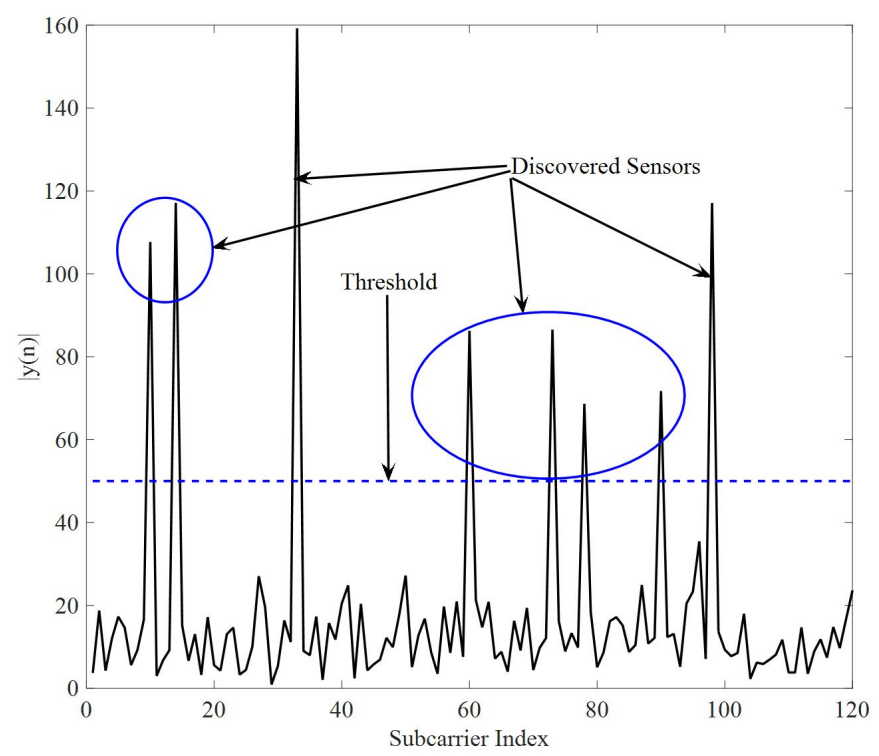

Figure 2.1: Detection of sensor nodes signature in frequency domain.

an example of calculated $\mathbf{y}$ at receiver. As shown the transmitted subcarrier are detectable defining proper threshold value. Although, it can be observed that due to the channel fading and noise, miss detection and false alarm are possible. Defining $\mathbf{z}=|\mathbf{y}|$, the probabilities of miss detection $\left(P_{m}\right)$ and false alarm $\left(P_{f}\right)$ in AWGN channel, respectively correspond to (see Appendix A.1 for proof):

$$
\begin{gathered}
P_{m}:=P\left(z_{n}<\lambda \mid \mathbf{s}^{(n)} \text { is within } \mathbf{r}\right)=1-Q_{1}\left(\frac{N}{\sigma \sqrt{L_{s}}}, \frac{\lambda}{\sigma \sqrt{L_{s}}}\right), \\
P_{f}:=P\left(z_{n}>\lambda \mid \mathbf{s}^{(n)} \text { is not in } \mathbf{r}\right)=e^{-\left(\frac{\lambda^{2}}{2 L_{s} \sigma^{2}}\right)},
\end{gathered}
$$

where $z_{n}=\left|y_{n}\right|$ and $\mathbf{s}^{(n)}$ is defined in 2.5), $N$ and $L_{s}$ are defined in 2.10 and $Q_{1}, \sigma^{2}$ and $\lambda$ represent the Marcum Q function, variance of additive white Gaussian noise and the value of threshold, respectively. There are two different approaches on 
selecting the value of $\lambda$. The first approach considers $\lambda$ a function of noise variance such as $\lambda=\lambda_{0} \sigma$, where $\lambda_{0}$ denotes a constant value and can be achieved using (2.11) and (2.12) for specific value of $P_{m}$ or $P_{f}$. This approach however, needs an estimation of the noise variance which requires a complex procedure specifically when the system is not synchronized. The second approach considers a constant value such as $\lambda=\lambda_{0}$. In Section 2.5 .2 the impacts of both approaches are investigated.

In the case of MPFS channels, the probability of miss detection $\left(P_{m}\right)$ is (see Appendix A.1 for proof):

$$
P_{m}=1-e^{-\frac{\lambda^{2}}{2 \sigma_{z}^{2}}}
$$

where $\lambda$ and $\sigma^{2}$ denote detection threshold and variance of additive noise, respectively. Moreover, $\sigma_{z}^{2}=\left(L_{s} \sigma^{2}+L N^{2} \sigma_{h}^{2}\right)$ where $N$ and $\sigma_{h}^{2}$ represent the length of transmitted signature and variance of Inphase and Quadrature components of channel impulse response, respectively. However, the probability of false alarm in 2.12 remains unchanged since there is no transmitted signature. As the ND procedure completes, the target node starts ToA estimation process for those sensor nodes which have been discovered. 


\subsubsection{ToA Estimation}

Similar to the ND problem, the target node can estimate the coarse ToA from the $L_{s}$ samples of received signal $\mathbf{r}$. Here, we only focus on coarse ToA estimation and ignore fine ToA estimation. The coarse ToA of the $n^{\text {th }}$ transmitted signature $\tau_{0}^{(n)}$ can be defined as a factor of sampling time $\left(T_{s}\right)$ such as $\tau_{0}^{(n)}=k^{*} T_{s}$, where $k^{*}$ is an integer value defined as the index of ToA. Therefore, the problem of ToA estimation is equivalent to the estimation of $k^{*}$. By discovering the $n^{\text {th }}$ sensor node signature $\left(\mathbf{s}^{(n)}\right)$ in ND process, the estimated ToA of its signature, $\hat{\tau}_{0}^{(n)}$ is calculated by $\hat{\tau}_{0}^{(n)}=\hat{k}^{*} T_{s}$, where $\hat{k}^{*}$ represents the estimation of $k^{*}$ and corresponds to:

$$
\hat{k}^{*}=\underset{k}{\operatorname{argmax}}\left\{\left|\left(\mathbf{s}^{(n)}\right)^{H} \mathbf{r}_{k: k+N-1}\right|\right\},
$$

where $\mathbf{r}_{k: k+N-1},|$.$| and (.)^{H}$ denote the $N$ consecutive samples of $\mathbf{r}$ defined at 2.1) from the $k^{t h}$ through the $(k+N-1)^{t h}$ sample, the absolute value and, the transposeconjugate operations, respectively. The target node needs to calculate the term $\left|\left(\mathbf{s}^{(n)}\right)^{H} \mathbf{r}_{k: k+N-1}\right|$ for all $1 \leq k \leq L_{s}$ and search for its maximum. Considering the $n^{\text {th }}$ sensor's signature, ideal channel $(h=1$ and $L=1)$, absence of noise and the 
ToA of the $n^{\text {th }}$ transmitted signature such that $\tau_{0}^{(n)}=k^{*} T_{s}$, it can be shown that:

$$
c_{k}=\left\{\begin{array}{l}
\left|\sum_{k^{\prime}=k^{*}}^{k+N-1} e^{\frac{-j 2 \pi n\left(k^{\prime}-k+1\right)}{N}} e^{\frac{j 2 \pi n\left(k^{\prime}-k^{*}+1\right)}{N}}\right|, k^{*}-N+1 \leq k \leq k^{*}, \\
\left|\sum_{k^{\prime}=k}^{k^{*}+N-1} e^{\frac{-j 2 \pi n\left(k^{\prime}-k+1\right)}{N}} e^{\frac{j 2 \pi n\left(k^{\prime}-k^{*}+1\right)}{N}}\right|, k^{*} \leq k \leq k^{*}+N-1, \\
0 \quad \text { otherwise },
\end{array}\right.
$$

where we define $c_{k}=\left|\left(\mathbf{s}^{(n)}\right)^{H} \mathbf{r}_{k: k+N-1}\right|$. Applying some mathematical manipulations, 2.15) corresponds to:

$$
c_{k}= \begin{cases}\left|e^{\frac{j 2 \pi n\left(k-k^{*}\right)}{N}} \sum_{k^{\prime}=k^{*}}^{k+N-1}\right| & k^{*}-N+1 \leq k \leq k^{*}, \\ \left|e^{\frac{j 2 \pi n\left(k-k^{*}\right)}{N}} \sum_{k^{\prime}=k}^{k^{*}+N-1}\right| & k^{*} \leq k \leq k^{*}+N-1, \\ 0 & \text { otherwise }\end{cases}
$$

which can be simplified to:

$$
c_{k}= \begin{cases}k-\left(k^{*}-N\right) & k^{*}-N+1 \leq k \leq k^{*}, \\ \left(k^{*}+N\right)-k & k^{*} \leq k \leq k^{*}+N-1, \\ 0 & \text { otherwise }\end{cases}
$$

where $N$ and $k^{*}$ denote the length of transmitted signature and the index of ToA, respectively. This indicates that the maximum value of $\mathbf{c}=\left[c_{1}, c_{2}, \ldots, c_{L_{s}}\right]$ occurs at $k=k^{*}$ for all $1 \leq k \leq L_{s}$. It can be shown that when there are more than one 
transmitted signatures within $\mathbf{r}$, the value of $\mathbf{c}$ would be none-zero for $k \leq k^{*}-N$ and/or $k^{*}+N \leq k$. This value is negligible when comparing to the maximum peak value of $\mathbf{c}$ which causes an error floor at high SNRs.

In the case of multi-path channels, following the same procedure such as $(2.15)-(2.17)$ leads to:

$$
c_{k}=\left\{\begin{array}{l}
\left(k-\left(k^{*}+l-N\right)\right) \mid \sum_{l=0}^{L-1} h_{l} e^{\frac{j 2 \pi n\left(k-k^{*}-l\right)}{N} \mid,} k^{*}-N+1 \leq k \leq k^{*}-1, \\
\left(k-\left(k^{*}+l-N\right)\right)\left|\sum_{l=0}^{L-1} h_{l} e^{\frac{j 2 \pi n\left(k-k^{*}-l\right)}{N} \mid}\right|, k^{*} \leq k \leq k^{*}+L-1, \\
\left(\left(k^{*}+l+N\right)-k\right)\left|\sum_{l=0}^{L-1} h_{l} e^{\frac{j 2 \pi n\left(k-k^{*}-l\right)}{N}}\right|, k^{*}+L \leq k \leq k^{*}+N+L-1, \\
0 \quad \text { otherwise, }
\end{array}\right.
$$

where $e^{\frac{j 2 \pi n\left(k-k^{*}-l\right)}{N}}$ represents the $(k-l)^{t h}$ element of $\mathbf{s}^{(n)}$ which is zero for $k-l \leq 0$. Here, unlike (2.17), there is no guarantee that the maximum value of $\mathbf{c}$ occurs at $k=k^{*}$ for all $1 \leq k \leq L_{s}$. However, the term $\left(k-\left(k^{*}+l-N\right)\right)$ in acts as a weight function which increases the probability of having the maximum value of $\mathbf{c}$ at $k=k^{*}$. Next subsection studies the improvement of this probability via increasing the number of transmitted sub-carriers within the sensor node's signature.

To evaluate the accuracy of the proposed technique, two different measures have been considered: $(1)$ the probability of error $\left(P_{e}\right)$ in the estimation of $k^{*}$, and (2.1) the Normalized Mean Square Error (NMSE) in ToA estimation. The probability of 
error that is defined as $P_{e}=P\left(\hat{k}^{*} \neq k^{*}\right)$, corresponds to $P_{e}=1-P_{c}$, and:

$$
P_{c}=P\left[c_{k^{*}}>c_{1}, c_{2}, \ldots, c_{k^{*}-1}, c_{k^{*}+1}, \ldots, c_{L_{s}-N}\right]
$$

where $P_{c}$ is the probability of correct estimation of $k^{*}$ and $c_{k}$ has defined in (2.15). To evaluate the performance of detected ToA theoretically an upper bound for $P_{c}$ is calculated (see Appendix A.2 for proof):

$$
\begin{array}{r}
P_{c} \leq \int_{0}^{\infty} \prod_{k=1}^{N-1}\left(1-Q_{1}\left(\frac{k}{\sqrt{(N-k) \sigma^{2}}}, \frac{c_{k^{*}}}{\sqrt{(N-k) \sigma^{2}}}\right)\right)^{2} \\
\left(1-e^{-\frac{c_{k^{*}}^{2}}{2 N \sigma^{2}}}\right)^{\left(L_{s}-3 N+1\right)} p_{c_{k^{*}}}\left(c_{k^{*}}\right) d c_{k^{*}},
\end{array}
$$

for:

$$
p_{c_{k^{*}}}\left(c_{k^{*}}\right)=\frac{c_{k^{*}}}{N \sigma^{2}} I_{0}\left(\frac{N c_{k^{*}}}{2 N \sigma^{2}}\right) e^{-\left(\frac{c_{k^{*}}^{2}+N^{2}}{2 N \sigma^{2}}\right)}
$$

where $N$ and $k^{*}$ are defined in (2.17), $I_{0}($.$) and \sigma^{2}$ represent the zero order Bessel function and the variance of complex Gaussian noise added to the received signal, respectively.

Considering $K$ independent estimations of $\tau^{(m)}$, NMSE corresponds to:

$$
N M S E=\frac{\sum_{n=1}^{K} \sum_{m=1}^{M}\left|\tau_{n}^{(m)}-\hat{\tau}_{n}^{(m)}\right|^{2}}{M K \tau_{\max }^{2}},
$$

where $\tau_{n}^{(m)}$ and $\hat{\tau}_{n}^{(m)}$ denote the ToA of the $m^{\text {th }}$ sensor node and its estimation at 
the $n^{\text {th }}$ iteration, respectively. Moreover, $\tau_{\max }, M$ and $K$ represent the propagation delay associated to the maximum range of sensor nodes, the number of target node's neighbors and the number of independent runs, respectively (see Table II).

\subsubsection{Impact of the Number of Sub-Carriers}

This section discusses the impact of increasing the number of sub-carriers $\left(N_{s}\right)$ allocated to each sensor's signature on the performance of ND and ToA estimation. The proposed ND procedure assumes that the target node would detect a sensor's signature if it detects at least one of the allocated sub-carriers to the signature of it's neighbors. It can be shown that the probabilities of miss detection is (see Appendix A.1 for proof):

$$
P_{m}=\left[1-Q_{1}\left(\frac{N}{\sigma \sqrt{L_{s}}}, \frac{\lambda}{\sigma \sqrt{L_{s}}}\right)\right]^{N_{s}}
$$

where $N, L_{s}, \lambda$ and $\sigma$ are defined in (2.11). However, the probability of false alarm in 2.12 remains unchanged since there is no transmitted signature.

The probability of correct ToA estimation in the case of one allocated sub-carriers

is discussed in 2.20 based on the value of $c_{k}=\left|\left(\mathbf{s}^{(n)}\right)^{H} \mathbf{r}_{k: k+N-1}\right|$, where $c_{k}$ is proposed in 2.18. Figure 2.2 sketches the value of $c_{k}^{\left(N_{s}\right)}=\prod_{p=1}^{N_{s}}\left|\left(\mathbf{s}^{(p)}\right)^{H} \mathbf{r}_{k: k+N-1}\right|$ for different numbers of transmitted sub-carriers $\left(N_{s}\right)$. Applying the same approach 
to $2.18, c_{k}^{\left(N_{s}\right)}$ leads to:

$$
c_{k}^{\left(N_{s}\right)}= \begin{cases}\left(k-\left(k^{*}+l-N\right)\right)^{N_{s}} \prod_{p=1}^{N_{s}} & \left|\sum_{p^{\prime}=1}^{N_{s}} \sum_{l=0}^{L-1} h_{l} e^{\frac{j 2 \pi n}{\left(p^{\prime}\right)\left(k-k^{*}-l\right)}}\right| \\ & k^{*}-N+1 \leq k \leq k^{*}-1, \\ \left(k-\left(k^{*}+l-N\right)\right)^{N_{s}} \prod_{p=1}^{N_{s}} & \left|\sum_{p^{\prime}=1}^{N_{s}} \sum_{l=0}^{L-1} h_{l} e^{\frac{j 2 \pi n}{\left(p^{\prime}\right)\left(k-k^{*}-l\right)}}\right| \\ & k^{*} \leq k \leq k^{*}+L-1, \\ \left(\left(k^{*}+l+N\right)-k\right)^{N_{s}} \prod_{p=1}^{N_{s}} & \left|\sum_{p^{\prime}=1}^{N_{s}} \sum_{l=0}^{L-1} h_{l} e^{\frac{j 2 \pi n}{\left(p^{\prime}\right)\left(k-k^{*}-l\right)}}\right| \\ & k^{*}+L \leq k \leq k^{*}+N+L-1, \\ & \text { otherwise, }\end{cases}
$$

As shown in Fig. 2.2, for $N_{s}=1$ (one sub-carrier in signature) the cost function has a triangular shape which is not an ideal form for ToA estimation. However, if the transmitted signature contains higher number of sub-carriers, $c_{k}^{\left(N_{s}\right)}$ converges to a delta function as $N_{s}$ increases. Therefore, considering $N_{s}$ transmitted sub-carriers, the proposed objective function in 2.14 can be revised to:

$$
\hat{k}^{*}=\underset{k}{\operatorname{argmax}}\left\{\prod_{p=1}^{N_{s}}\left|\left(\mathbf{s}^{(n, p)}\right)^{H} \mathbf{r}_{k: k+N-1}\right|\right\},
$$

where $\mathbf{s}^{(n, p)}$ represents the $p^{\text {th }}$ transmitted sub-carrier by the $n^{\text {th }}$ sensor node and $\mathbf{r}$ is defined in (2.1). A large number of sub-carrier allocation methods for OFDMA have 
been proposed in the literature. Here, the proposed form in [103] is incorporated in which pairs of allocated signatures such as $\left\{k, k / N_{T}-2\right.$ or $\left.k / N_{T}+2\right\}$ are selected where $k$ and $N_{T}$ are random sub-carriers and the total number of sub-carriers in hand, respectively.

Considering $N_{s}$ transmitted sub-carriers within the sensor node's signature, 2.20 changes to (see Appendix A.2 for proof):

$$
\begin{gathered}
P_{c}=\int_{0}^{\infty} \ldots \int_{0}^{\infty}\left(P\left[\prod_{p=1}^{N_{s}} c_{k^{*}}^{(p)}>\prod_{p=1}^{N_{s}} c_{1}^{(p)}\right]\right)^{\left(L_{s}-3 N+1\right)} \\
\left(\prod_{k=1}^{N-1} P\left[\prod_{p=1}^{N_{s}} c_{k^{*}}^{(p)}>\prod_{p=1}^{N_{s}} c_{k^{*}-N+k}^{(p)}\right]\right)^{2} \\
f_{c_{k^{*}}^{(1)}}\left(c_{k^{*}}^{(1)}\right) \ldots f_{c_{k^{*}}^{\left(N_{s}\right)}}\left(c_{k^{*}}^{\left(N_{s}\right)}\right) d c_{k^{*}}^{(1)} \ldots d c_{k^{*}}^{\left(N_{s}\right)}
\end{gathered}
$$

Here , $P\left[\prod_{p=1}^{N_{s}} c_{k^{*}}^{(p)}>\prod_{p=1}^{N_{s}} c_{1}^{(p)}\right]$ is quite close to one since $\left(\prod_{p=1}^{N_{s}} c_{k^{*}}^{(p)}\right)$ is much larger than $\left(\prod_{p=1}^{N_{s}} c_{1}^{(p)}\right)$. However, the dominant part in 2.26 is $P\left[\prod_{p=1}^{N_{s}} c_{k^{*}}^{(p)}>\prod_{p=1}^{N_{s}} c_{k^{*}-N+k}^{(p)}\right]$ which leads to the upper bound proposed in (63). Increasing $N_{s}$ in (62) increases $\gamma=\frac{\prod_{p=1}^{N_{s}} c_{k^{*}}^{(p)}}{\prod_{p=2}^{N_{s}} c_{k^{*}-N+k}^{(p)}}$ which decreases $Q_{1}\left(\frac{k}{\sqrt{(N-k) \sigma^{2}}}, \frac{\gamma}{\sqrt{(N-k) \sigma^{2}}}\right)$ and increases the proposed upper bound for $P_{c}$. 


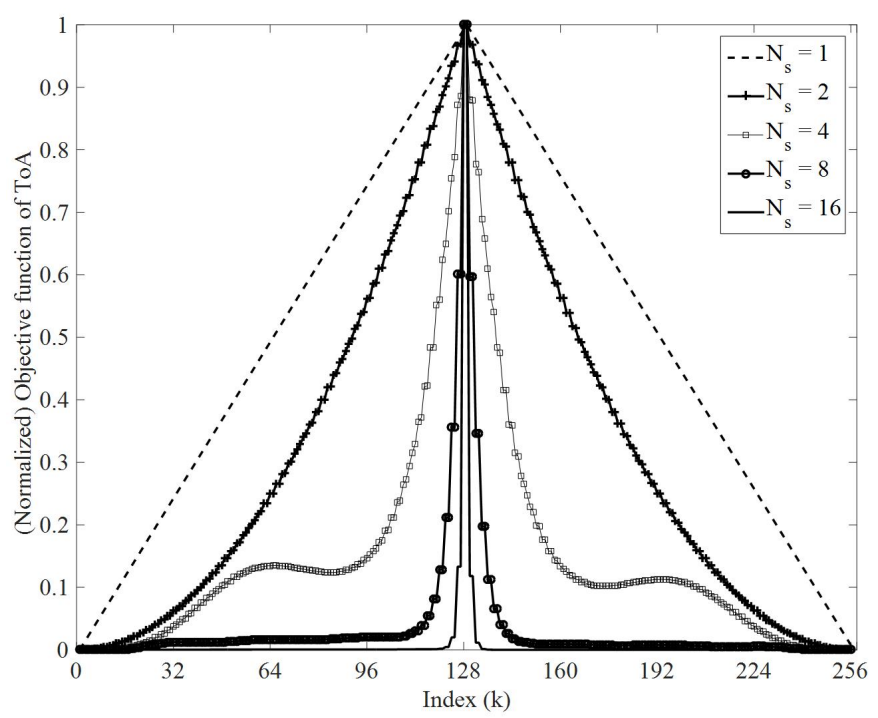

Figure 2.2: Objective function for ToA estimation for different numbers of transmitted sub-carrier(s).

\subsection{Experimental Challenges}

\subsubsection{Energy Efficiency and Scalability}

In order to investigate the energy efficiency of the proposed technique, the consumed energy (RF transceiver and processing) of the entire ND and ToA estimation is calculated and compared to the existing state-of-the-art techniques for ND and ToA estimation, considering a popular sensor node platform (the CC2500 transceiver [104] and MSP430 processor [105]). Here, the multi-band chirp signal is selected as the transmitter employing the time-slot based approaches [89], [90] at MAC layer. 
To this end, the total value of consumed current $(\mathrm{mA})$ by target/neighbor node is calculated applying the following equations:

$$
I_{t}=T_{t x} I_{t x}+T_{r x} I_{r x}+T_{a} I_{a}+T_{i} I_{i}
$$

where $I_{t}$ represents the total value of consumed current $(\mathrm{mA})$ and $I_{t x}, I_{r x}, I_{a}$ and $I_{i}$ denote the current consumption for the radio transmission and reception, and the processor at active and idle modes, respectively. Moreover, $T_{t x}, T_{r x}, T_{a}$ and $T_{i}$ represent the time period (normalized to $1 \mathrm{sec}$ ) of the radio transmission and reception, and the period of processor's active and idle modes, respectively. The current consumption of the CC2500 transceiver [104] and MSP430 processor [105] are considered as follow: $I_{t x}=22, I_{r x}=14, I_{a}=0.23$ at $Q_{p}=1 \mathrm{MHz}$ and $I_{i}=0.009$ all in $\mathrm{mA}$. Table 2.1 presents the total time for transmission and reception and the total number of real-value instructions (multiplication, summation or comparison). In order to calculate the time periods, we used the length of the transmitted and received signal multiplied by sampling time $\left(T_{s}\right)$. For the processor active time we exploited $T_{a}=\left(Q / Q_{p}\right) T_{s}$ where $Q$ and $Q_{p}$ represent the total required instructions defined in Table 2.1 and the total instructions per second of the processor $Q_{p}=$ $1 M H z$, respectively. Simulation results (Section 2.5.4) confirm that the total value of consumed current $\left(I_{T}=I_{t}^{(\text {target })}+M I_{t}^{(\text {neighbor })}\right)$ exploiting the proposed approach is much less than the multi-band chirp signal that uses the time-slot based MAC such as [89], [90]. 
Table 2.1

Active transmission $\left(T_{x}\right)$ and reception $\left(T_{r}\right)$ and the total number of real-value instructions at target (Tar.) and neighbor (Nei.) nodes for ND and ToA estimation of the proposed method (Pro.) and multi-band chirp exploiting the time-slot based approaches

\begin{tabular}{|c|c|c|c|}
\hline Method & $T_{x}$ & $T_{r}$ & $Q$ \\
\hline \hline Pro. (Tar.) & $N T_{s}$ & $2 L_{s} T_{s}$ & $10 N L_{s}+10 M N N s(L s-N)$ \\
Pro. (Nei.) & $N T_{s}$ & $L_{s} T_{s}$ & $(L s-N)(10 N-1)$ \\
[78] (Tar.) & $N N_{s} T_{s}$ & $N^{2} N_{s} T_{s}$ & $N((L s-N)(10 N N s-1)+N)$ \\
[78] (Nei.) & $N N_{s} T_{s}$ & $L_{s} T_{s}$ & $(L s-N)(10 N-1)$ \\
\hline
\end{tabular}

Another important factor for any protocol at WSNs is the scalability. The scalability in WSNs indicates the ability of the proposed technique to support the network expansion (by node density or quantity) [106]. Here, a PHY layer technique is proposed for ND and ToA estimation which should be combined with a sub-carrier allocation algorithm at its MAC layer. The proposed technique could be applied to any network size (any node density of number) considering a proper sub-carrier allocation (unique allocation to all neighbors of any sensor) algorithm. In other words, the scalability of the proposed method should be evaluated by the scalability of the exploited sub-carrier allocation approach. The sub-carrier allocation for OFDMA based wireless communications is well discussed in the literature. These algorithms cannot be applied to WSN due to its limitations such as unknown location of each sensor node and the deficiency of base stations. Nevertheless, it is straightforward to infer that the probability of sub-carriers collision imposed by network expansion is increased by increasing the total number of sub-carriers $N_{T}$. However, beside the bandwidth limitations, increasing the value of $N_{T}$ to support the network expansion 
increases the energy consumption of the proposed method as shown in Table 2.1. Therefore, it can be concluded that the scalability of the proposed method leads to a tradeoff between the probability of sub-carriers collision (performance/scalability) and the network energy efficiency.

\subsubsection{OFDMA Limitations}

Despite the advantageous (such as, multi-path/user efficiency), OFDMA has some disadvantageous such as sampling time offset (SFO), carrier frequency offset (CFO) and peak to average power ratio (PAPR), which severely degrade the performance of proposed approach. Here, we aim to discuss these problems and propose possible solutions.

\subsubsection{SFO and CFO}

Similar to the OFDM in wireless communications, the orthogonality of transmitted sub-carriers is the key feature of the proposed method which can be removed in the presence of the SFO and/or CFO. Synchronization is the most popular approach to alleviate the imposed affects by $\mathrm{SFO}$ and $\mathrm{CFO}$. Here, we propose the time domain (before multiplying the received signal by DFT matrix, W) synchronization applying the efficient method proposed in [103] and [107] prior to the ND and ToA 
estimation. Applying this technique, all sensor nodes should transmit a common (no ND required) tone (sub-carrier) prior to the ND and ToA estimation which enables the SFO and CFO estimation. The fast convergence and high estimation accuracy of this technique offers an efficient solution for the synchronization in WSNs.

\subsubsection{PAPR}

The PAPR is originated from the simultaneous transmission of different sub-carriers with the same (proposed method) or different (OFDMA at wireless communications) amplitude [101]. Unlike the OFDMA at wireless communication, no simultaneous sub-carriers transmission is necessary considering a pre-defined delay such as $D=$ $N$. This only increases the transmission period $\left(T_{t x}\right)$ of neighbor nodes which is negligible compare to the consumed energy by the processor. Therefore, the target node can apply the same ND to discover transmitted sub-carriers, however, the ToA estimation objective function is revised to:

$$
\hat{k}^{*}=\underset{k}{\operatorname{argmax}}\left\{\prod_{p=1}^{N_{r}}\left|\left(\mathbf{s}^{(n, p)}\right)^{H} \mathbf{r}_{k+(p-1) D: k+N+(p-1) D-1}\right|\right\}
$$

where $N_{r}$ and $D$ are number of sub-carrier transmission and the pre-defined delay of each sub-carrier, $\mathbf{s}^{(n, p)}$ represents the $p^{\text {th }}$ transmitted sub-carrier by the $n^{\text {th }}$ sensor node and $\mathbf{r}$ is defined in 2.1 . 


\subsection{Simulation Results and Discussion}

Simulations are conducted to investigate the performances of the proposed ND and ToA estimation methods. The probabilities of miss detection and false alarm of neighbor sensor nodes are calculated to evaluate the performance of the proposed method for neighbor discovery problem. Moreover, for ToA estimation, the NMSE of estimated delay is proposed. The system performances are evaluated at both AWGN and MPFS channels considering different numbers of independent taps. Moreover, the impact of allocated sub-carriers is investigated in a sperate sub-section. Finally, the energy efficiency and the scalability of the proposed method is studied by simulation of consumed energy for the proposed technique and the probability

of sub-carrier(s) collision, respectively. In the following sub-section, we investigate the parameters that are used for system model simulation. The performance of the proposed ND method and ToA estimation are discussed in Sections 2.5.2 and 2.5.3, respectively.

\subsubsection{Simulation Parameters and Methods}

In this sub-section, the details of simulated system model which is used for performance analysis are introduced. Table 2.2 shows the definitions and the values of 
parameters which are used to simulate the system model. Furthermore, the definition of signal to noise ratio (SNR) corresponds to:

$$
S N R=\frac{\left|\sum_{m=1}^{M_{T}} \gamma^{(m)}\left(\mathbf{h}^{(m)}\right)^{H} \mathbf{s}^{(m)}\right|^{2}}{N \sigma^{2}\left|\sum_{m=1}^{M_{T}} \gamma^{(m)}\left(\mathbf{h}^{(m)}\right)^{H} \mathbf{h}^{(m)}\right|^{2}},
$$

where $N, M_{T}, \gamma^{(m)}$ are defined in 2.1 and $\mathbf{h}^{(m)}, \mathbf{s}^{(m)}$ and $\sigma^{2}$ denote the channel impulse response between the target node and the $m^{\text {th }}$ sensor node, the $m^{\text {th }}$ sensor node's signature and noise power, respectively. Furthermore, (. $)^{H}$ and |.| represent the transpose-conjugate and absolute value operations, respectively. Here, a MATLAB based simulation platform consisting $M_{T}$ sensor nodes with maximum range of $R$, exploited uniformly within an operation is considered. In order to measure the performance of the proposed method, a target node surrounded by $M$ neighbor nodes is considered where: 1 . each sensor transmits its allocated signature proposed in (2.4) as soon as it receives the request signal. 2. The transmitted signal is passed through the AWGN/MPFS channel considering the complex (circularly-symmetric) normal and the Rayleigh distributions for the additive noise and channel taps amplitude, respectively. 3. The target node accumulates the $L_{s}$ (see (2.3) ) samples of the received signal based on the system model described in (2.1) to initiates the ND and ToA estimation as discussed at Section 2.3. 4. For ND the probabilities of false alarm and miss detection have been simulated using the proposed definitions in (2.11) and 2.12), respectively, applying $K$ independent run of Monte Carlo method. 
Table 2.2

Simulation parameters and applied values

\begin{tabular}{|c|l|l|}
\hline Sym. & Definition & Value \\
\hline \hline$T_{s}$ & Sampling time & $6.25 n s$ \\
$N$ & Length of signature & 128 \\
$N_{T}$ & Total number of sub-carriers & 128 \\
$N_{s}$ & Sub-carriers in signature & $1 / 2 / 4 / 8 / 16$ \\
$\Delta f$ & Sub-carrier spacing & $1 /\left(N T_{s}\right)=1.25 M H z$ \\
$M_{T}$ & Total No. of sensor nodes & 128 \\
$M$ & Number of neighbors & $1 / 2 / 4 / 8$ \\
$R$ & Maximum range & $1000 \mathrm{~m}$ \\
$L_{s}$ & Length of received signal & $2 R /\left(c T_{s}\right)+N=1200$ \\
$L$ & Taps of channel & $1 / 3 / 7$ \\
$T_{m}$ & Channel delay spread & $T_{s}$ \\
$K$ & No. of independent runs & $1 e 4 / 1 e 5$ \\
\hline
\end{tabular}

5. The probability of error and NMSE of ToA estimation incorporates the proposed definitions in 2.19 and 2.22 proceeding the ND within the same run.

\subsubsection{Neighbor Discovery Performance}

In Figs. 2.3 and 2.4 the probabilities of miss detection $\left(P_{m}\right)$ and false alarm $\left(P_{f}\right)$ are evaluated assuming flat and MPFS channels. Simulation results are consistent with the prediction made by theory presented in 2.12 and 2.13. In this simulation, a variable threshold as a function of noise power such as $\lambda=0.7 N \sigma$ is considered. As shown, in Fig. 2.3, $P_{m}$ decreases by increasing the number of channel taps. 


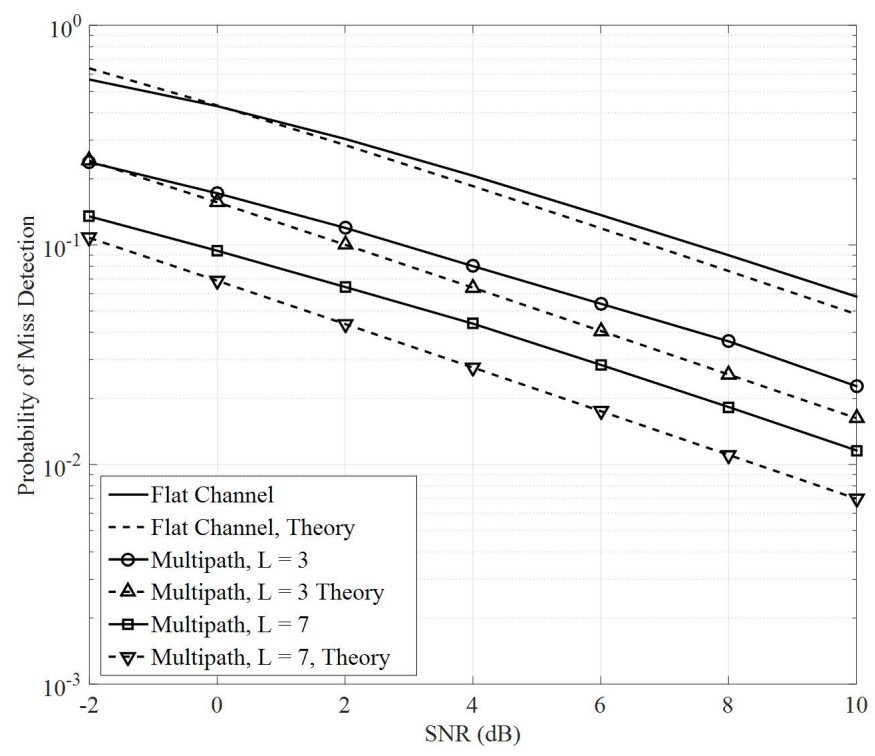

Figure 2.3: Probability of miss detection for neighbor discovery in MPFS channel using variable threshold.

This outcome is predictable in the case of multi-path channels, as each tap has an independent probability of fading across all taps and therefore, the probability of fading across all of them is less than each of them individually. This result is consistent with the theoretical value of $P_{m}$ derived in (2.11) and (2.13). However, applying $\lambda=0.7 N \sigma$ into 2.12 leads to the constant false alarm rate of $P_{f}=$ $e^{-(0.7 N)^{2} / 2 L_{s}}$ for AWGN and MPFS channels as depicted in Fig. 2.4. To investigate the impact of fixed threshold value on detection performance, the simulation of Figs. 2.5 and 2.6 are conducted. Figs. 2.5 and 2.6 show $P_{m}$ and $P_{f}$ for two fixed values of threshold, respectively. Here, the case of AWGN is considered to compare the values of $P_{m}$ and $P_{f}$ theoretically and via simulations. As shown, by changing the value of threshold, the desired value of $P_{m}$ and $P_{f}$ for a specific SNR value can be maintained. 


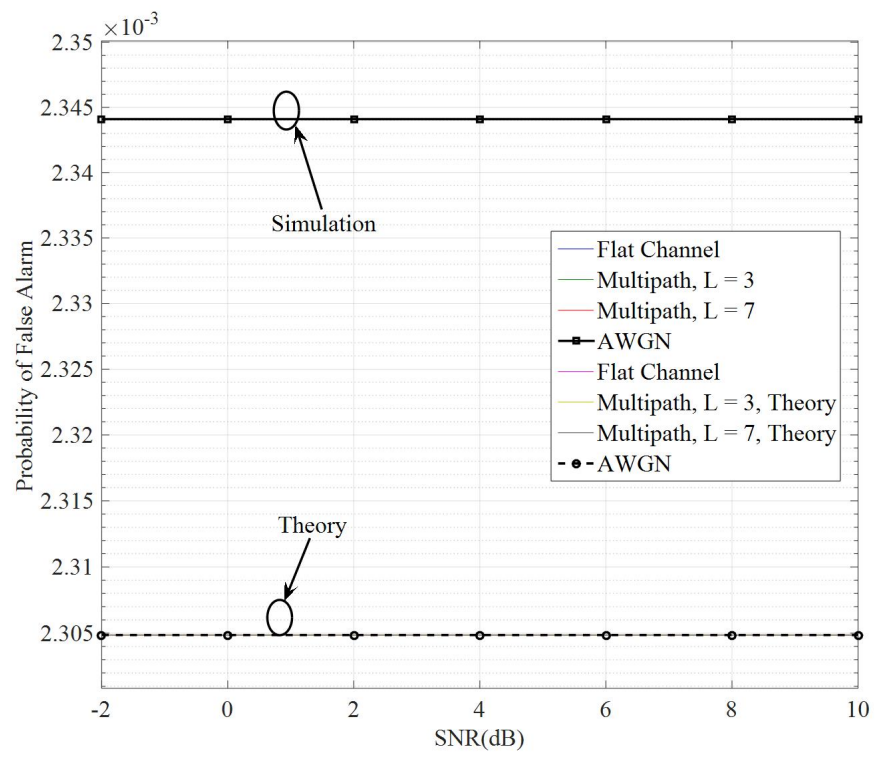

Figure 2.4: Probability of false alarm for neighbor discovery in MPFS channel using variable threshold.

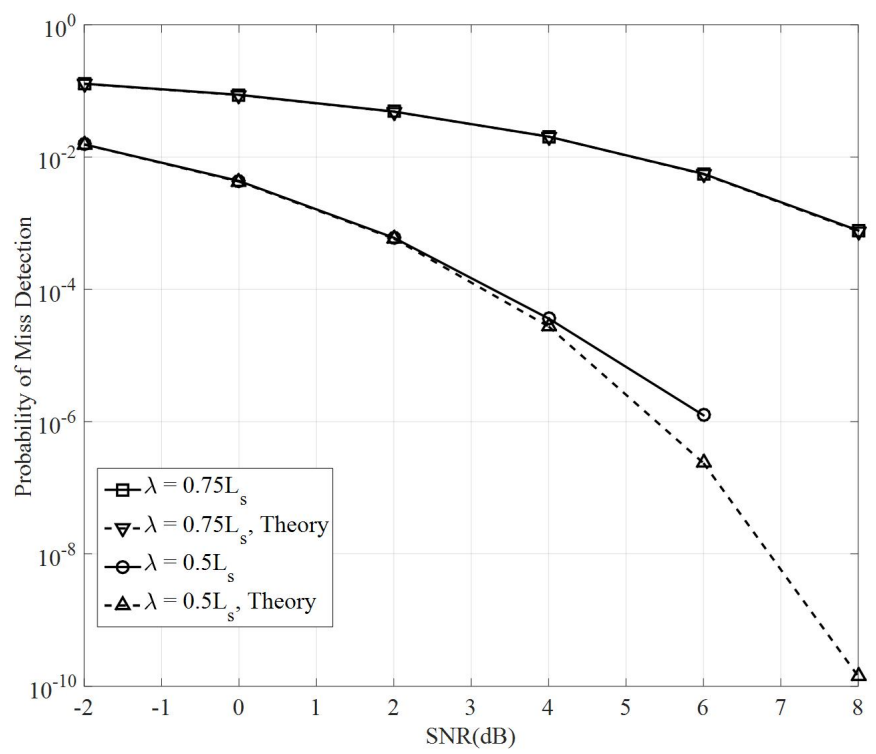

Figure 2.5: Probability of miss detection for neighbor discovery in AWGN channel using fixed value threshold.

Figs. 2.7 and 2.8 investigate the impact of the number of transmitted sub-carriers within the sensor node's signature on the ND procedure applying the variable threshold $(\lambda=0.7 N \sigma)$. As shown in Fig. 2.7, the probability of miss detection decreases 


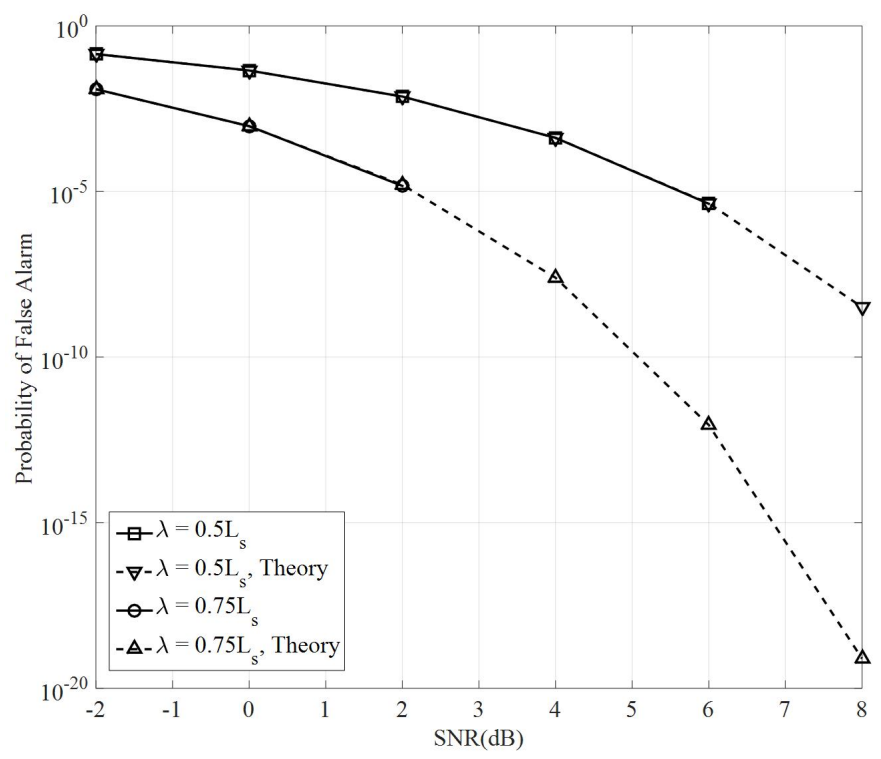

Figure 2.6: Probability of false alarm for neighbor discovery in AWGN channel using fixed value threshold.

significantly as the number of allocated sub-carriers increases. This also can be inferred from 2.23). However, as shown in Fig. 2.8 the probability of false alarm is the same as (2.12) since no signature is considered within the received signal.

\subsubsection{ToA Estimation Performance}

To investigate the value of estimated ToA, NMSE of estimated ToA has been depicted in Fig. 2.9 in AWGN channel applying different numbers of neighbors $(M)$. Increasing $M$ causes two changes to be observed in the NMSE curve (see Fig. 2.9). First, the NMSE increases as $M$ increases, second, the slope of the NMSE curve decreases as SNR increases. In Section 2.3.2, we mentioned that the term 


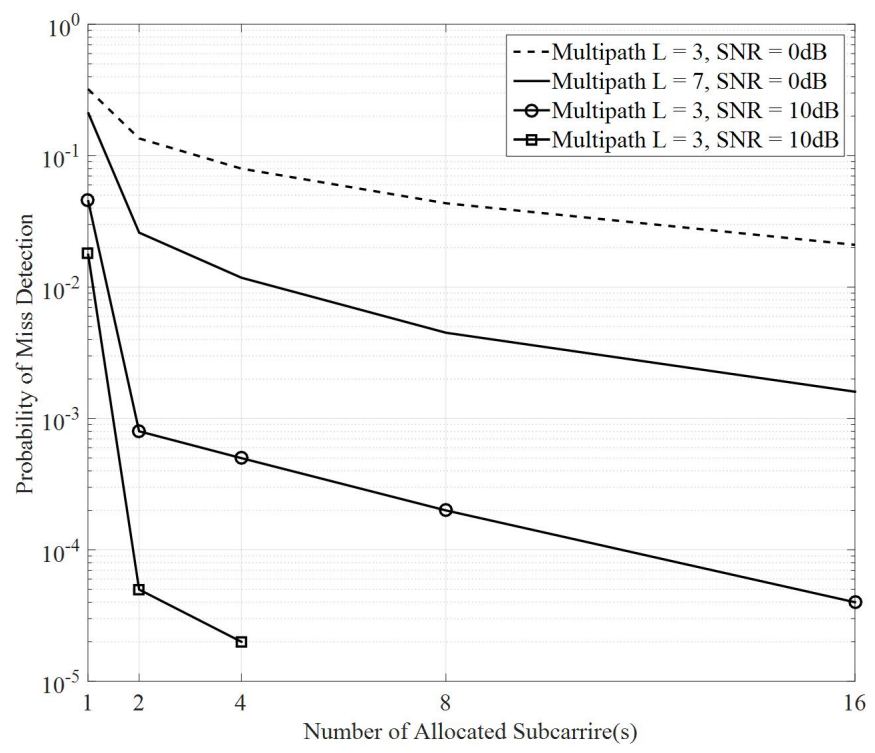

Figure 2.7: Effect of allocated sub-carriers on the probability of miss detection.

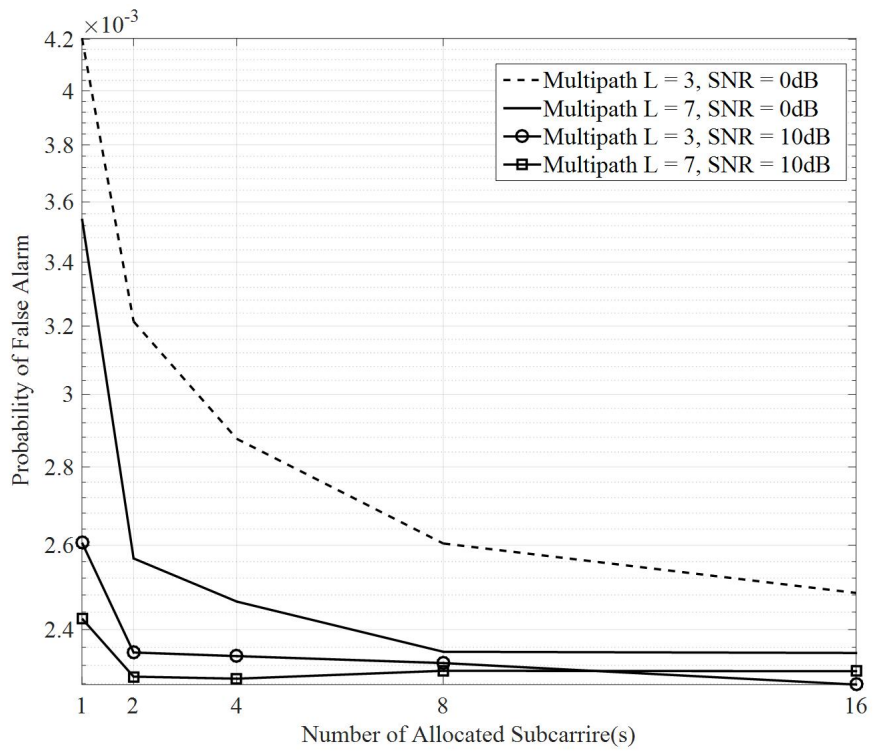

Figure 2.8: Effect of allocated sub-carriers on the probability of false alarm.

$\left|\left(\mathbf{s}^{(n)}\right)^{*} \mathbf{r}_{k: k+N-1}\right|$ in 2.14$)$ can be a none zero value for $k \leq k^{*}-N$ and/or $k^{*}+N \leq k$.

Here, the impact of this term on increasing the value of NMSE and decreasing the slope of NMSE curve for high SNR values is observed. 
Figure 2.10 shows the NMSE of ToA estimator in flat and MPFS channels with $L$ taps. In this simulation, two different values of $M$ are considered to compare the impact of multiuser ToA estimation in MPFS channels. Here, it is observed that multi-path effect of channel improves the performance of ToA estimator. As mentioned in the previous sub-section, the probability of group fading of all taps is much less than each of them individually. However, comparing Figs. 2.9 to 2.10 for $M=1$ indicates that AWGN still has the best performance.

As mentioned earlier in Section 2.3.2, in the case of multi-path channels the probability that the maximum value of (2.18) occurs at $k=k^{*}$ increases when the value of $\left(k-\left(k^{*}+l-N\right)\right)$ for $k=k^{*}$ is much larger than its value for $k \neq k^{*}$. This only can be achieved by increasing the number of transmitted sub-carriers where (2.18) changes to 2.25) and therefore the value of $\left(k-\left(k^{*}+l-N\right)\right)$ increases to $\left(k-\left(k^{*}+l-N\right)\right)^{N_{s}}$, as discussed in Section 2.3.3. Figure 2.11 depicts the probability of error $\left(P_{e}\right)$ in MPFS channels $(L=7$ taps) applying different numbers of sub-carriers. As shown, the probability of error improves as the transmitted signature employs a larger number of sub-carriers. In Fig. 2.12, the NMSE of the proposed ToA estimation in the presence of MPFS channels $(L=3$ and 7$)$ is depicted considering different values of $N_{s}$. Comparing the proposed results in Fig. 2.10, it is observed that the performance of ToA estimator in 2.27) improves tremendously by increasing the number of transmitted sub-carriers, even in the case of multi-path channels. Moreover, Figs. 2.12 and 2.13, compare the performance of the proposed 


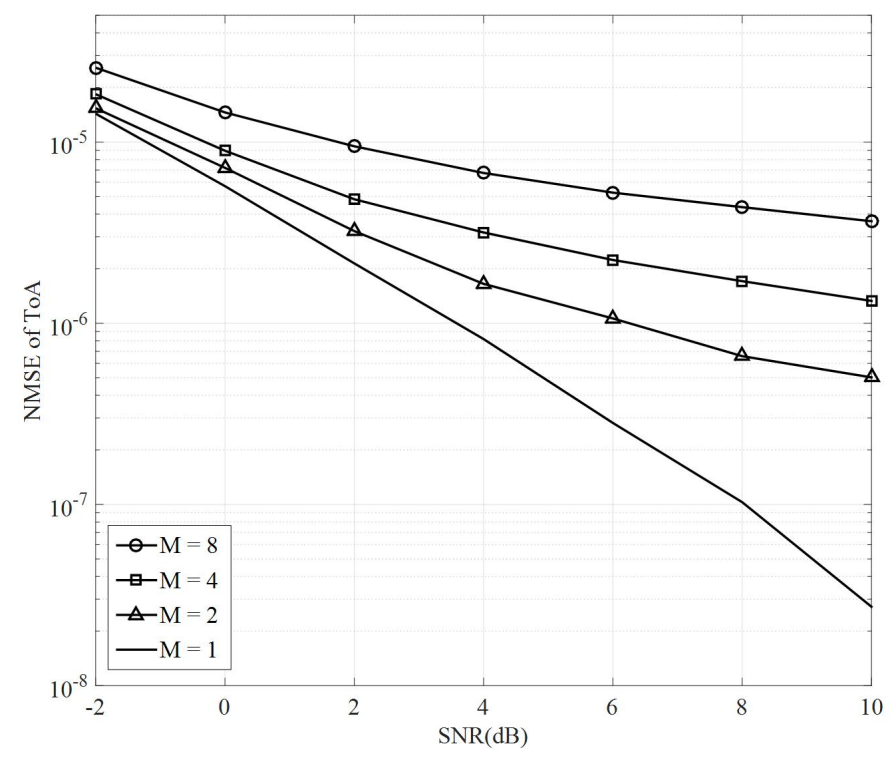

Figure 2.9: Normalized mean square error (NMSE) of ToA estimation in AWGN channel considering different numbers of neighbor nodes.

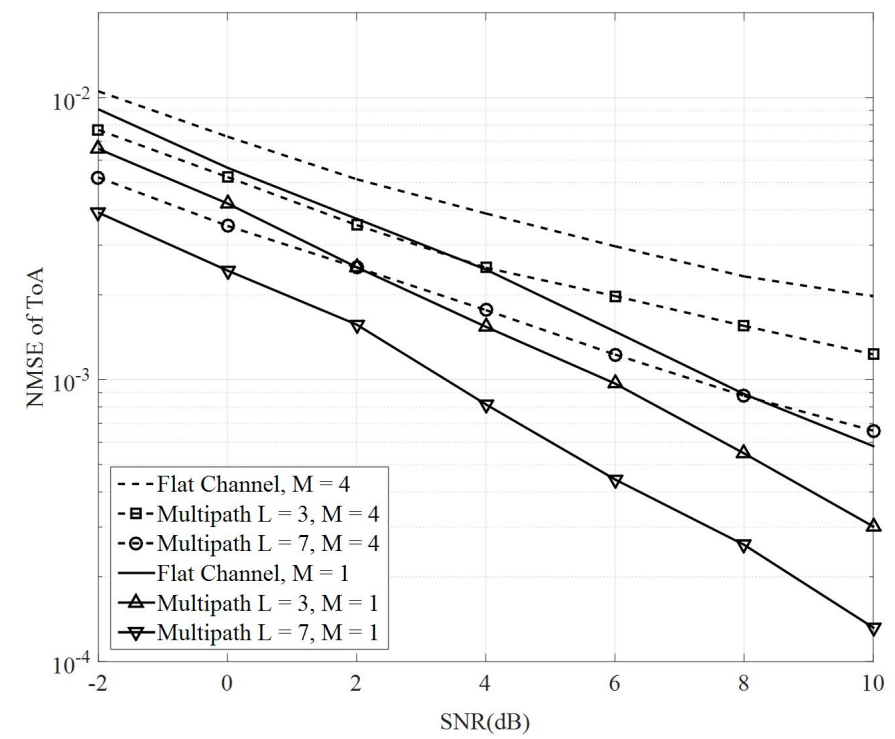

Figure 2.10: Normalized mean square error (NMSE) of ToA estimation in multi-path channel considering different numbers of neighbor nodes.

method to the multi-band chirp signal [78, where the transmitted signals have the same length and enjoy the same bandwidth. Moreover, no information of channel impulse response is available. As shown, the proposed method outperforms the 


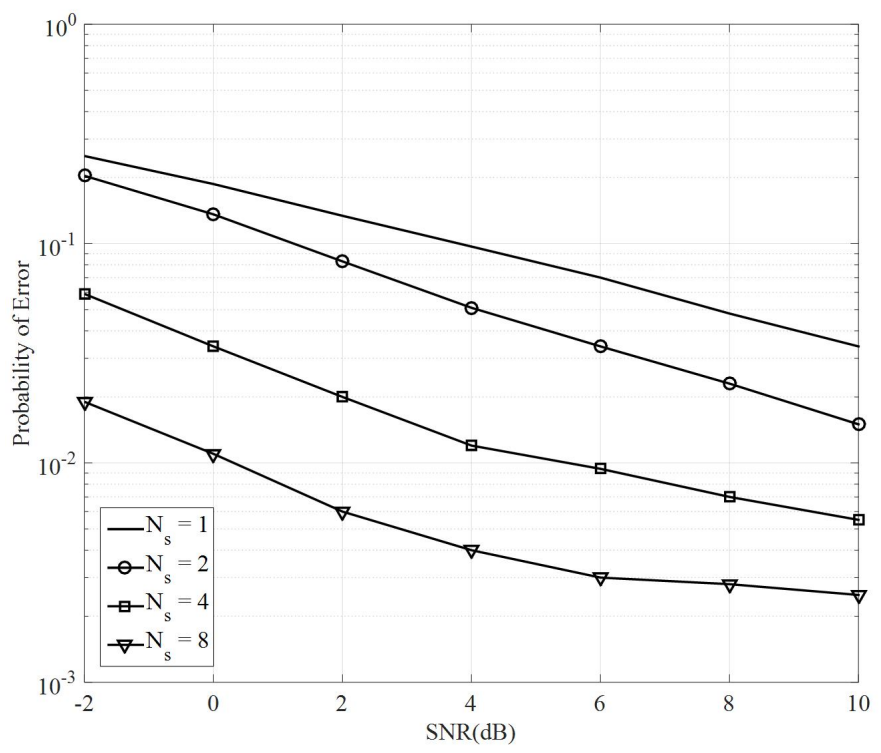

Figure 2.11: Impact of increasing the number of transmitted sub-carriers in probability of error in ToA estimation.

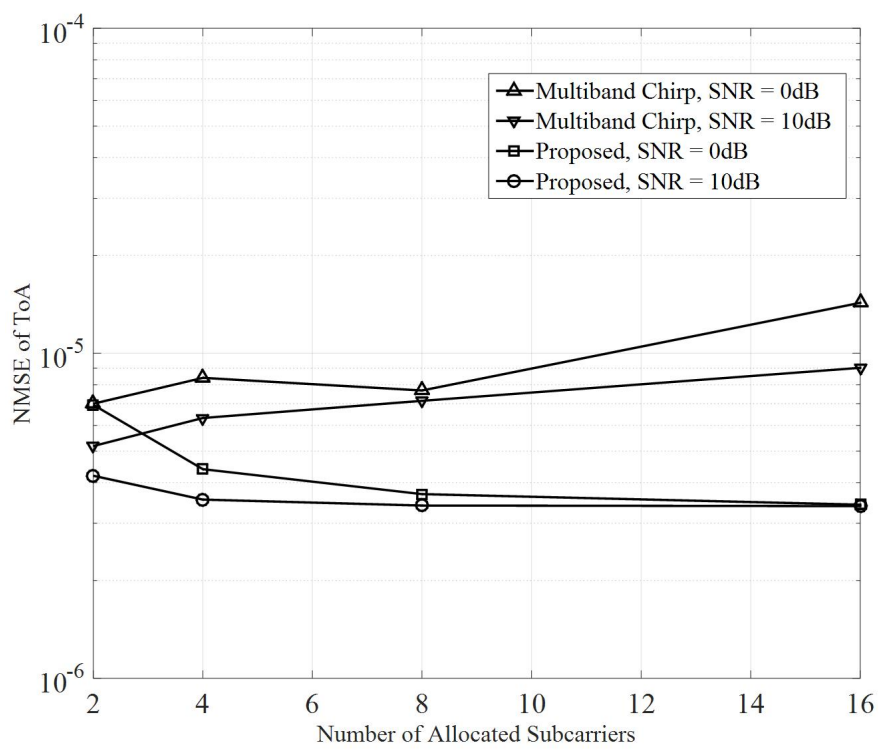

Figure 2.12: Impact of increasing the number of transmitted sub-carriers in NMSE of ToA estimator v.s. multi-band chirp signal

multi-band chirp specifically when the allocated bandwidth increases. This is due to the independence of the proposed sensor's signature length to the allocated subcarrier (bandwidth); however, for optimum performance of multi-band chirp signal 


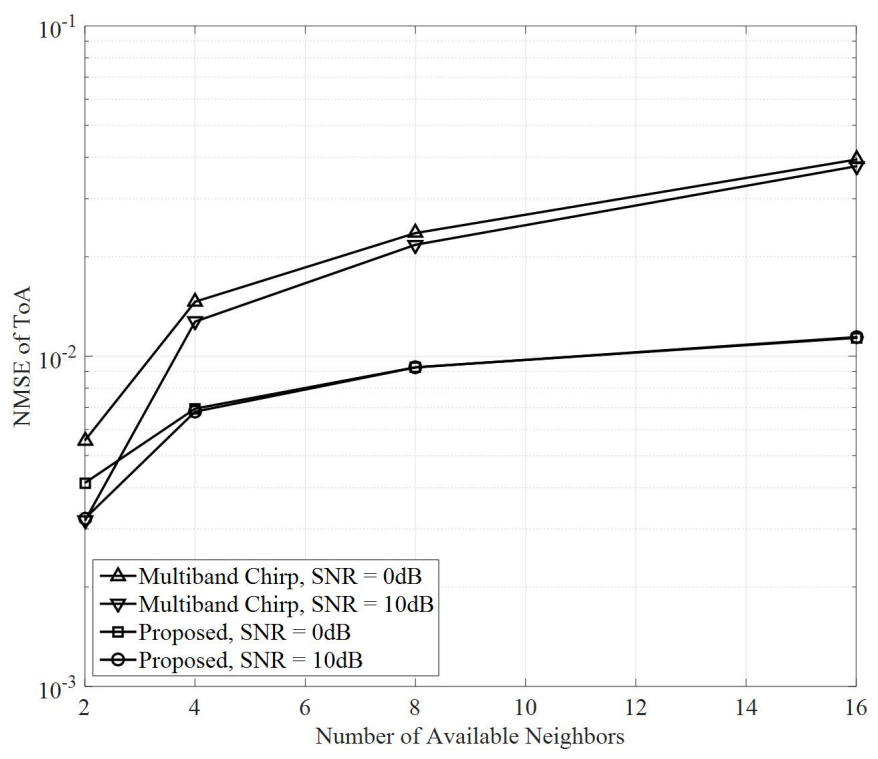

Figure 2.13: Normalized mean square error (NMSE) of ToA estimation in multi-path channel considering different numbers of neighbor nodes, v.s. multi-band chirp signal

the length of transmitted signal should be increased by extending the transmitted bandwidth. Fig. 2.13 compares the performance of the proposed method to the multi-band chirp in MPFS channel for different numbers of neighbor nodes $(M)$. Here, as expected, the performance of ToA estimator degrades by increasing the number of neighbor nodes due to increasing the probability of collision, however the ToA NMSE of the proposed method increases by lower rate compared to the multi-band chirp signal. 


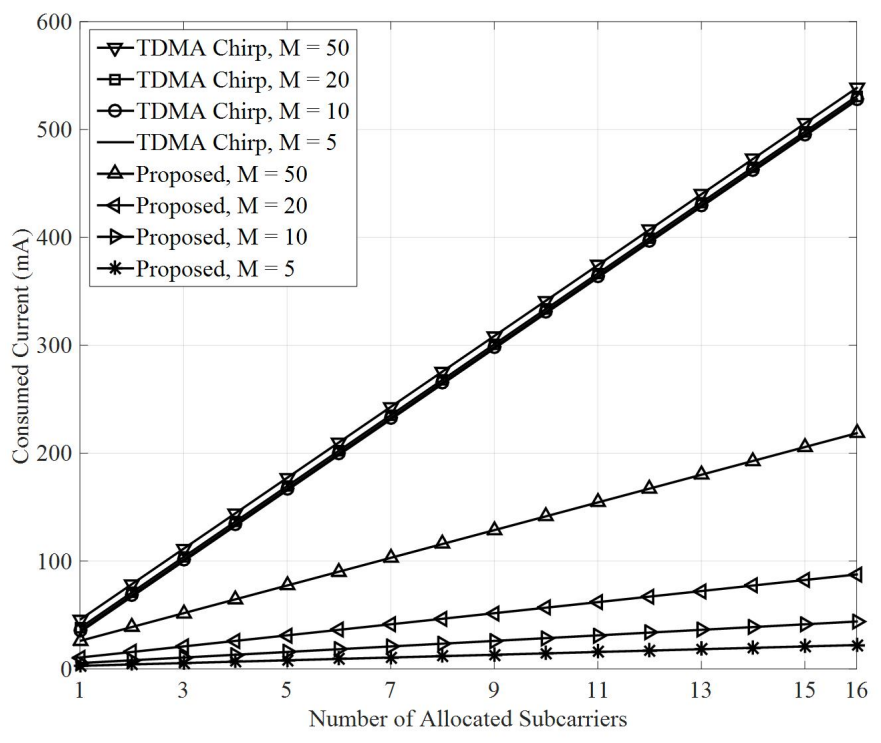

Figure 2.14: Total current consumption value $(\mathrm{mA})$, applying the TDMA based multi-band chirp and proposed method

\subsubsection{Energy Efficiency and Scalability Analysis}

Figure 2.14 depicts the total value of consumed current $\left(I_{T}\right)$ for the multi-band chirp signal [78] employing the time-slot based approaches [89], [90] at MAC layer and proposed method exploiting (27). As shown the consumed energy depends on the number of the allocated sub-carriers $\left(N_{s}\right)$ to each sensor node (the available bandwidth in multi-band chirp) and the number of available (detected) neighbor nodes. Comparing these figures indicates the energy efficiency of the proposed method compare to the multi-band chirp signal [78] employing the time-slot based approaches [89], 90] at MAC layer and proposed method. 


\subsection{Conclusions and Future Works}

\subsubsection{Conclusion}

This chapter proposed joint ND and ToA estimation for WSNs in MPFS channel. By unique allocation of an OFDMA sub-carrier as each sensor node's signature, we propose ND and ToA estimation methods which alleviates the affects of collision of signals transmitted from neighbor nodes and in turn increase the performance. The proposed method is energy efficient as it avoids multiple transmissions and receptions which is utilized in traditional ND and ToA methods. Moreover, the proposed method reduces channel MPFS affects for both ND and ToA estimation. To investigate the performance of $\mathrm{ND}$, the probabilities of miss detection and false alarm were evaluated. Moreover, the performance of ToA estimation was investigated by calculating the probability of error and normalized mean square error, theoretically and via simulations. Performance analysis confirms that the proposed methods for ND and ToA estimation have acceptable performance in MPFS channel specifically when the allocated sub-carriers to each sensor node are increased. Therefore, we propose a feasible solution for ND and ToA estimation in WSNs because it only needs one transmission and reception and offers high performance ToA estimation 
even in low SNR regimes and MPFS channels. Moreover, it is also an appropriate method for dynamic ND and ToA estimation as it is fast compared with the traditional methods. Simulation results indicates the energy efficiency and the scalability of the proposed method, however, the scalability is achievable via increasing the number of available sub-carrier or performing an advanced sub-carrier allocation which is considered for future study.

\subsubsection{Future Works and Discussion}

Although, the very fact that increasing the number of transmitted sub-carriers entails increasing the bandwidth and computational complexity, proposes a tradeoff between scalability and performance, and bandwidth/energy efficiency. However, considering the availability of wide bandwidth for new generations of wireless communication such as Millimeterwave or 5G, makes the proposed method a feasible solution for ND, coarse ToA estimation and finally localization in new generations of WSNs. 


\section{Chapter 3}

\section{High Resolution ToA Estimation}

\section{via Optimal Waveform Design ${ }^{1}$}

\subsection{Introduction}

Designing a waveform with good autocorrelation properties (close to delta function) has been discussed in literature [108] [109, 110, 111]-[112]. In [109] authors propose several cyclic algorithms such as CA-pruned (CAP), CAN (CA-new) and weighted CAN (WeCAN) where the latter offers the best performance in terms of minimum integrated sidelobe level (ISL) of the waveform's autocorrelation. Exploiting the

\footnotetext{
${ }^{1}$ The material contained in this chapter is submitted for publication in IEEE Transaction on Communications, July, 2016
} 
proposed waveform, one can estimate ToA with a resolution that is limited to the sampling interval. Higher resolution is achievable via decreasing sampling interval, however, this increases the waveform bandwidth which is not feasible and/or affordable in many wireless communications and wireless sensor network applications. Some prior works [92], propose fine ToA estimation in addition to traditional coarse ToA approaches, however, these techniques consider a known channel impulse response (CIR) or prior knowledge of statistical model for multipath channels [113] which are not feasible prior to time synchronization.

Despite the impact of bandwidth on the performance of designed waveform in ToA estimation, a few works discuss the problem of ToA estimation exploiting bandlimited waveforms. In [108], authors propose the stop-band CAN (SCAN) which aims to design a band-limited waveform with good autocorrelation properties. Exploiting this technique one can design a waveform with good autocorrelation properties considering smaller sampling interval (higher ToA resolution) at fixed bandwidth, however, in the proposed technique the ISL increases while a feasible attenuation in stop-band is interested. Authors in [114 and [115] propose different criteria for sparse spectrum limitation, however, these techniques cannot impose acceptable attenuation into stop-band while the waveform still performs good autocorrelation in terms of ISL.

In this chapter, a novel approach is proposed to improve the resolution of ToA 
estimation for a fixed available bandwidth in the presence of unknown multipath frequency selective (MPFS) channels. First, the low resolution of ToA estimator imposed by limited bandwidth of the designed waveform is addressed by proposing the maximum rising level detector (MRLD) technique via oversampling and parallel correlation paths. Here, ToA resolution improves via finding the path corresponding to the maximum rising level of the matched filters output of distinct correlation paths at their maximum absolute values. The idea of exploiting oversampling to improve the resolution of ToA [116]-[118], range [119] and synchronization [120] has been discussed in the literature. Many of these techniques use oversampling to increase the direct search resolution which cannot be applied to correlation based techniques because of sidelobe levels increase. In the MRLD technique the sidelobe levels of matched filter output corresponding to the samples generated by oversampling process are extremely larger than sidelobe levels of matched filter output of the original samples. However, the rising level created by oversampling at the autocorrelation center would be high as well. To this end, a novel technique exploiting the trustregion algorithm [121] is proposed which designs waveforms with the aforementioned properties via minimizing the weighted ISL (WISL) objective function.

The performance of the proposed technique is evaluated by simulating the estimated ToA mean square error (MSE), and compared to the state-of-the-art approach of SCAN [108] considering the same bandwidth. Moreover, the ToA Cramer-Rao lower bound (CRLB) is calculated in the presence of MPFS as evaluation benchmark. 
Simulation results verify the feasibility of the proposed technique compared to techniques such as SCAN specially over unknown MPFS channels. Furthermore, it is observed that increasing the oversampling rate or equivalently the number of correlation paths, improves the ToA resolution. In addition, increasing the oversampling rate reduces the gap between MSE of ToA and its corresponding CRLB imposed by lack of fine ToA estimation. Finally, the complexity and performance of the proposed technique is discussed theoretically and via simulations for different numbers of correlation paths and waveform lengths.

The rest of this chapter is organized as follow. Section 3.2 introduces the system model and problem formulation. The proposed MRLD technique and the algorithm for waveform design is presented in Section 3.3 . Section 3.4 represents the simulation results and discussions, and finally Section 3.5 concludes the chapter.

\subsection{System Model and Problem Formulation}

To perform ToA estimation, receiver should detect the arrival of the transmitted signal instantaneously, and measure the signal propagation time with respect to a 
reference time. The system model for the baseband received signal corresponds to:

$$
r(t)=\left\{\begin{array}{cc}
\sum_{l=0}^{L-1} h_{l} s\left(t-\tau_{l}\right)+v(t), \tau_{0} \leq t \leq \tau_{L-1}+(N-1) T_{s} \\
v(t), & \text { elsewhere }
\end{array}\right.
$$

where $s(t), h_{l}, \tau_{l}$ and $v(t)$ represent the transmitted baseband signal with length $N$, gain and delay of the $l^{\text {th }}$ tap of wireless channel impulse response, and additive, white, zero mean Gaussian noise, respectively. Applying the proposed baseband received signal in (3.1) to the analog to digital converter (ADC), with the sampling frequency of $f_{s}=1 / T_{s}$, where $T_{s}$ is the sample interval of the baseband signal, leads to:

$$
r(k)=\left\{\begin{array}{c}
\sum_{l=0}^{L-1} h_{l} s\left(k T s-\tau_{l}\right)+v\left(k T_{s}\right), k_{\tau_{0}} \leq k \leq k_{\tau_{L-1}}+N-1 \\
v\left(k T_{s}\right), \quad \text { elsewhere }
\end{array}\right.
$$

where $k_{\tau_{l}}$ is the closest integer number to $\tau_{l} / T_{s}$, and $L, h_{l}, \tau_{l}$ and $N$ are defined in (3.1), and $s\left(k T_{s}\right)$ and $v\left(k T_{s}\right)$ represent the $k^{\text {th }}$ sample of the transmitted waveform and additive zero mean Gaussian noise, respectively.

The ToA estimation is equal to the estimation of the time delay corresponding to the received signal from the shortest path, $\tau_{0}$. Considering $\tau_{0}=k_{\tau_{0}} T_{s}+\delta_{\tau_{0}}$, ToA can be introduced via coarse $\left(k_{\tau_{0}} T_{s}\right)$ and fine $\left(\delta_{\tau_{0}}\right)$ components. In correlation based ToA techniques, the problem of ToA estimation is represented by estimating coarse-ToA 
via solving the following objective function:

$$
\hat{k}_{\tau_{0}}=\underset{k}{\operatorname{argmax}}\left|\sum_{n=k}^{k+N-1} r_{n} s_{N+n-k}^{*}\right|^{2}
$$

where $r_{n}$ and $s_{n}^{*}$ represent the $n^{\text {th }}$ sample of received signal defined in 3.2, and the complex conjugate of the transmitted waveform, respectively. This technique proposes a ToA resolution limited to the sample interval $T_{s}$. Given $\left|\sum_{n=k_{0}}^{N} r_{k} s_{N+n-k_{\tau_{0}}}^{*}\right|^{2}>\left|\sum_{n=k}^{N} r_{k} s_{N+n-k}^{*}\right|^{2}, \forall k, k \neq k_{\tau_{0}}$, the maximum of 3.3 occurs at $k=k_{\tau_{0}}$. Considering a constant energy signal, the aforementioned inequality holds via minimizing of the energy of sidelobe levels, $\left|\sum_{n=k}^{N} r_{k} s_{N+n-k}^{*}\right|^{2}, \forall k, k \neq k_{\tau_{0}}$. This is equivalent to minimization of ISL defined as [108, 109]:

$$
I S L=\sum_{k=1}^{N-1}\left|y_{k}\right|^{2}, \text { for }, \quad y_{k}=\sum_{n=k}^{N-1} s_{n} s_{N+n-k}^{*}
$$

where $s_{n}$ and $s_{n}^{*}$ represent the $n^{\text {th }}$ sample of the transmitted waveform and its complex conjugate, respectively. Therefore, minimization of ISL defined in (3.4) leads to a waveform with good autocorrelation properties and consequently, lower ToA estimation error. In [109], the weighted-ISL (WISL) is introduced where sidelobe levels are weighted to form a desired autocorrelation, such that:

$$
W I S L=\sum_{k=1}^{N-1} w_{k}^{2}\left|y_{k}\right|^{2}, w_{k} \geq 0
$$


where $y_{k}$ is the $k^{t h}$ autocorrelation sidelobe defined in (3.4), and $w_{k}$ represents its corresponding weight. Exploiting such criteria allows the designer to minimize the sidelobes around to the center of autocorrelation, in order to minimize the interference imposed by multipath channels [109].

Improving ToA estimation via optimal bandlimited signal is defined as designing a waveform with minimum WISL and bandwidth. However, in [108, 109], it is shown that minimization of (W)ISL, leads to a sequence with near wight spectrum which uniformly includes all frequencies within $\left[-f_{s} / 2, f_{s} / 2\right]$, where $f_{s}$ represents the sampling frequency. This indicates that limiting signal bandwidth to any spectrum lower than $\left[-f_{s} / 2, f_{s} / 2\right]$, dramatically incenses the (W)ISL which leads to ToA estimation error.

Next section introduces the maximum rising level detector (MRLD) technique which tremendously improves the low resolution of ToA estimation imposed by bandwidth limitations of the designed waveform via oversampling and parallel processing(see section 3.1). 


\subsection{Proposed Technique}

In this section enhancing the ToA resolution using the MRLD technique is proposed. Then, a novel procedure for optimization of weighted ISL (WISL) is proposed which leads to a waveform with very high rising level at its autocorrelation.

\subsubsection{Maximum rising level Detector}

Figure 3.1 shows the proposed transmitter (a), and receiver (b) block diagrams corresponding to the proposed MRLD technique. Applying the baseband received signal in (3.1) to the analog to digital convertor (ADC) with sampling time interval $\tilde{T}_{s}=T_{s} / M$, where $T_{s}$ represents the sample interval of the transmitted waveform, leads:

$$
r\left(k^{\prime} \tilde{T}_{s}\right)=\left\{\begin{array}{l}
\sum_{l=0}^{L-1} h_{l} s\left(k^{\prime} \tilde{T}_{s}-\tau_{l}\right)+v\left(k^{\prime} \tilde{T}_{s}\right), \\
M k_{\tau_{0}}+k_{\delta} \leq k^{\prime} \leq M k_{\tau_{L-1}}+k_{\delta_{L-1}}+N-1 \\
v\left(k^{\prime} \tilde{T}_{s}\right), \quad \text { elsewhere }
\end{array}\right.
$$

where $s\left(k \tilde{T}_{s}\right), r\left(k \tilde{T}_{s}\right)$ and $v\left(k \tilde{T}_{s}\right)$ represent the samples of transmitted and received signals, and zero-mean complex additive Gaussian noise at $t=k \tilde{T}_{s}$, and $\left\{h_{l}\right\}_{l=0}^{L-1}$ denote the complex value channel impulse response (CIR). Furthermore, $M, k_{\tau_{l}}$ and 
$k_{\delta_{l}}$ are the oversampling rate and sample indices corresponding to the coarse and fine of $l^{\text {th }}$ path delay, respectively. Reformulating the ToA equation $\left(\tau_{0}=k_{\tau_{0}} T_{s}+\delta_{\tau_{0}}\right)$ in terms of new sample time, $\tilde{T}_{s}$ leads to:

$$
\tau_{0}=\left(M k_{\tau_{0}}+k_{\delta}\right) \tilde{T}_{s}+\tilde{\delta}_{0}
$$

where $k_{\tau_{0}}$ and $k_{\delta_{0}}$ are the sample indices corresponding to the coarse and fine ToA, respectively. Moreover, $\tilde{\delta}_{0}$ represents the remnant of fine ToA such that $\tilde{\delta}=\delta_{\tau_{0}}-k_{\delta} \tilde{T}_{s}$ for $0 \leq k_{\delta} \leq M-1$.

The proposed MRLD receiver oversamples the baseband received signal with rate $M$, then segregates the output of ADC into $M$ parallel correlation paths each corresponding to a consecutive sample time offset $0, \tilde{T}_{s}, \ldots,(M-1) \tilde{T}_{s}$. Exploiting this technique, the sequence of existing samples in the $m^{\text {th }}$ correlation path corresponds to the samples observed at times $\left[(m-1) \tilde{T}_{s},(M+m-1) \tilde{T}_{s},(2 M+m-1) \tilde{T}_{s}, \ldots\right], \forall m=$ $0,1, \ldots, M-1$.

Assuming zero-ISI pulse shaping at transmitter, the $k^{\text {th }}$ sample at the $m^{\text {th }}$ correlation path can be represented by:

$$
r_{m}\left(k T_{s}\right)=\left\{\begin{array}{c}
\sum_{l=0}^{L-1} h_{l} s\left((M k+m) \tilde{T}_{s}-\tau_{l}\right)+v\left((M k+m) \tilde{T}_{s}\right), k_{\tau_{0}} \leq k \leq k_{\tau_{L-1}}+N \\
v\left((M k+m) \tilde{T}_{s}\right), \text { elsewhere }
\end{array}\right.
$$


where $M$ is the number of correlation path or oversampling rate, and $h_{l}, s\left(k \tilde{T}_{s}\right)$, and $v\left(k \tilde{T}_{s}\right)$ are defined in 3.6 . Here, estimating ToA is equivalent to discovering the index corresponding to $\tau_{0}$ in terms of new sampling time, i.e. $\left(M k_{\tau_{0}}+k_{\delta}\right)$. Applying the sequence of each correlation path to the matched filter of transmitted waveform $\left(s^{*}(N-n)\right)$, and taking the absolute value, offers $M$ possible candidates for coarse ToA of $M k_{\tau_{0}} \tilde{T}_{s}=k_{\tau_{0}} T_{s}$. However, higher resolution $\left(k_{\delta} \tilde{T}_{s}\right)$ and its corresponding coarse ToA are achievable via searching over path numbers to reveal the one contains the closest samples to the transmitted waveform or its convolution with CIR. Figure 3.2 depicts the correlation levels corresponding to the MRLD receiver for $M=4$, considering the transmitted waveform with very high rising level at its autocorrelation center (see section 3.3.2 for waveform design). Here, (without loss of generality) it is assumed $\tau_{0}=\left(k_{0}+0.75\right) T_{s}$ where $\tau_{0}, T_{s}$ and $k_{0}$ represent the ToA, sampling interval and an arbitrary integer. Exploiting the traditional correlation techniques, the estimated ToA would be $\hat{\tau}_{0}=\left(k_{0}+1\right) T_{s}$. Considering (3.8), the sequence of existing samples in the third correlation path corresponds to:

$$
\begin{aligned}
r_{3}\left(k T_{s}\right)=h_{0} s\left((4 k+3) \tilde{T}_{s}-\tau_{0}\right) & +\sum_{l=1}^{L-1} h_{l} s\left((4 k+3) \tilde{T}_{s}-\tau_{l}\right) \\
& +v\left((4 k+3) \tilde{T}_{s}\right), \quad k_{0} \leq k \leq k_{\tau_{L-1}}+N
\end{aligned}
$$

where $h_{l}, s\left(k \tilde{T}_{s}\right)$ and $v\left(k \tilde{T}_{s}\right)$, and $r_{m}\left(k T_{s}\right)$ are defined in 3.6 and 3.8 , respectively. 

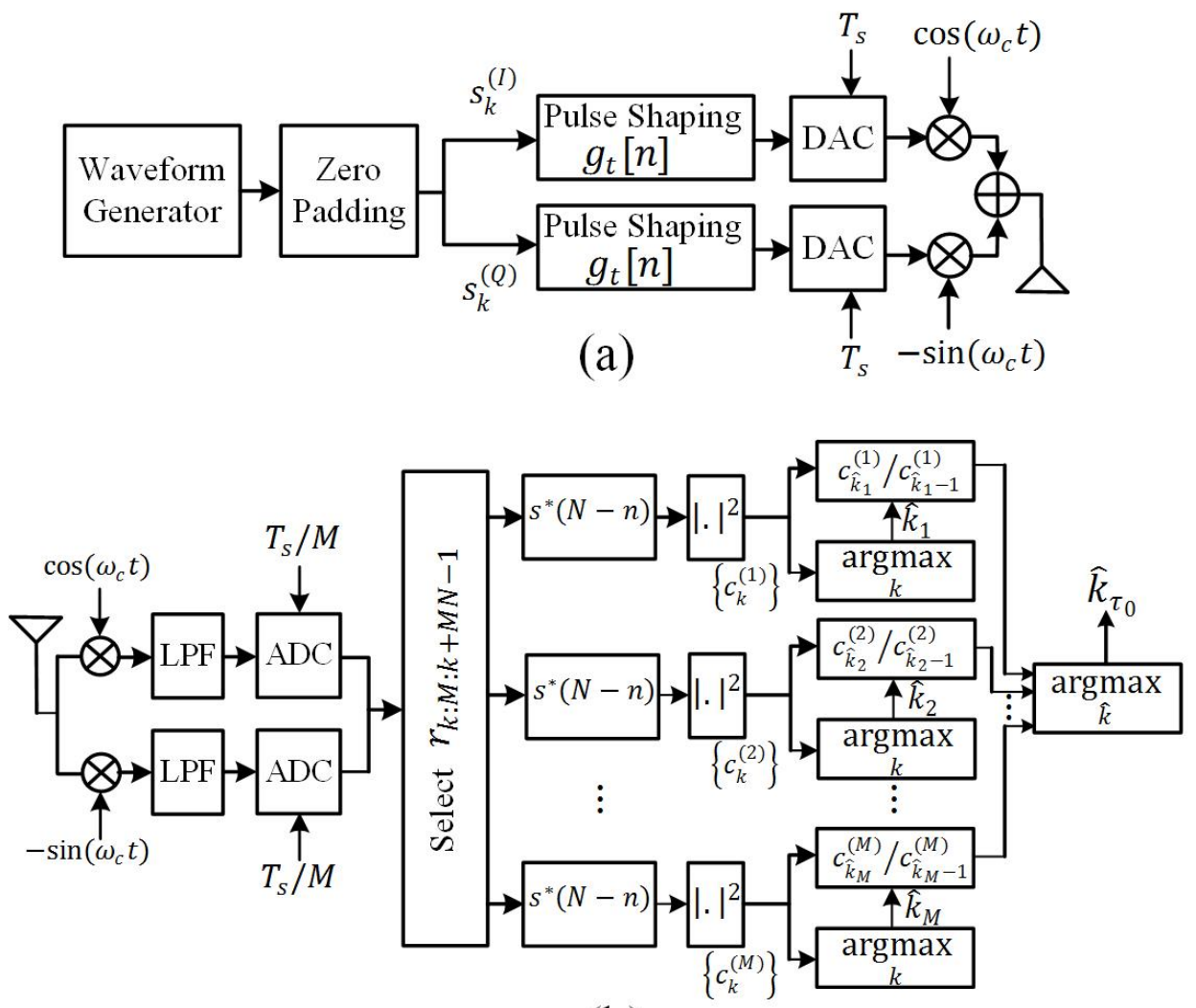

(b)

Figure 3.1: The block diagram of the proposed MRLD transceiver.

Substituting $\tau_{0}$ and $\tilde{T}_{s}$ with $\left(T_{0}+0.75\right) T_{s}$ and $T_{s} / 4$, respectively, leads to:

$$
\begin{aligned}
r_{3}\left(k T_{s}\right)=h_{0} s\left(\left(k-k_{0}\right) T_{s}\right) & +\sum_{l=1}^{L-1} h_{l} s\left((4 k+3) \tilde{T}_{s}-\tau_{l}\right) \\
& +v\left((4 k+m) \tilde{T}_{s}\right), \quad k_{0} \leq k \leq k_{\tau_{L-1}}+N
\end{aligned}
$$

The proposed sequence in $(3.10)$ is the convolution of the transmitted waveform and CIR which maximizes the matched filter output at $k=k_{0}$. Although, the matched filter outputs corresponding to the other pathes is maximum at the same index, 
however, their corresponding rising levels of the offered indexes are much smaller than the rising level of the third path (5, 17 and $15 \mathrm{~dB}$ shown in Fig. (3.2)-(a), (b) and (d) compared to $275 \mathrm{~dB}$ as shown in Fig. (3.2)-(c)). This is the result of designed waveform with a very high rising level proposed in section 3.2 , where the sidelobe levels corresponding to the correlation of the designed waveform matched filter with samples generated by oversampling are extremely larger than the sidelobe levels corresponding to its autocorrelation. Thus, the MRLD technique is efficiently implementable based on three simple steps: (1) searching for the peak of correlation levels at each path using the transmitted waveform matched filter $\left(s^{*}(N-n)\right),(2)$ measuring the rising level corresponding to the revealed peaks at each path, (3) searching for the maximum among the measured rising level. Therefore, the path containing the maximum rising level $\left(m^{*}\right)$, among $M$ available correlation paths is achievable via:

$$
\begin{gathered}
m^{*}=\underset{m}{\operatorname{argmax}}\left(\frac{c_{\hat{k}_{m}}^{(m)}}{c_{\hat{k}_{m}-1}^{(m)}}\right), \\
\text { s.t. } c_{\hat{k}_{m}}^{(m)}=\underset{k}{\operatorname{argmax}}\left|\sum_{n=k}^{k+N-1} r_{m}\left(n T_{s}\right) s_{N+n-k}^{*}\right|^{2},
\end{gathered}
$$

where $r_{m}\left(k T_{s}\right)$ and $s_{N-k}^{*}$ are the $k^{t h}$ samples of received sequence at $m^{\text {th }}$ correlation path and the matched filter to the transmitted waveform, respectively. Moreover, $M$ and $N$ represent the number of correlation paths or oversampling rate, and the length of received signal, respectively. Using (3.11), the estimated ToA can be 


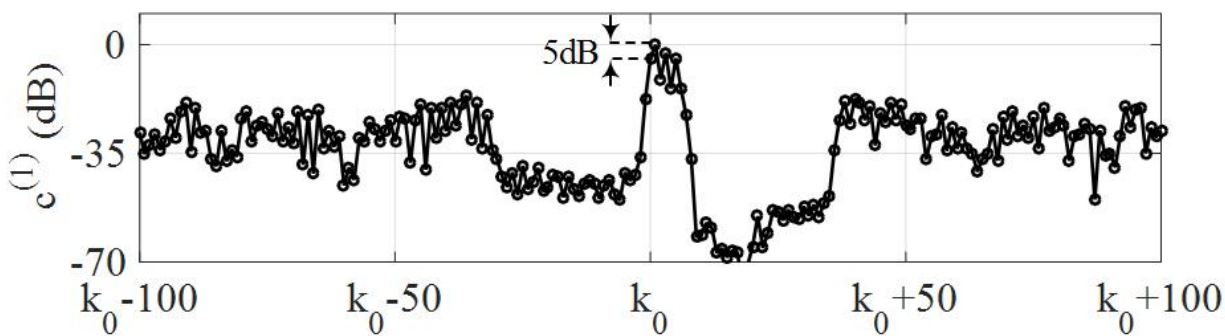

(a)

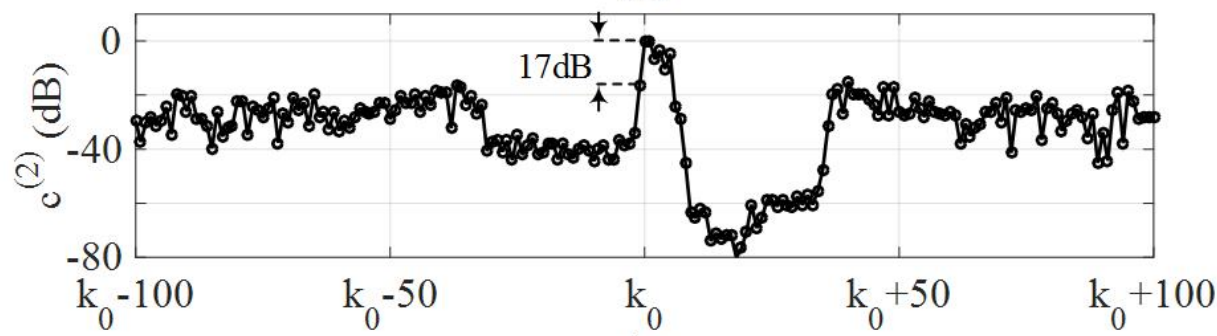

(b)

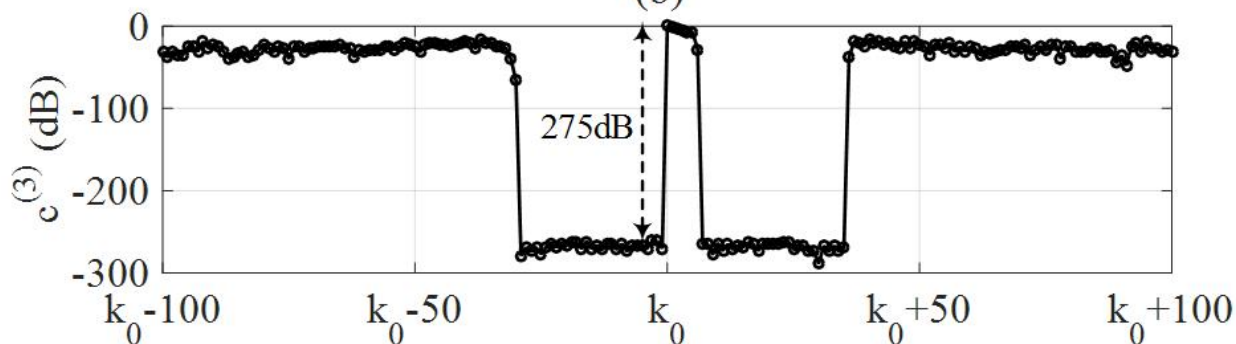

(c)

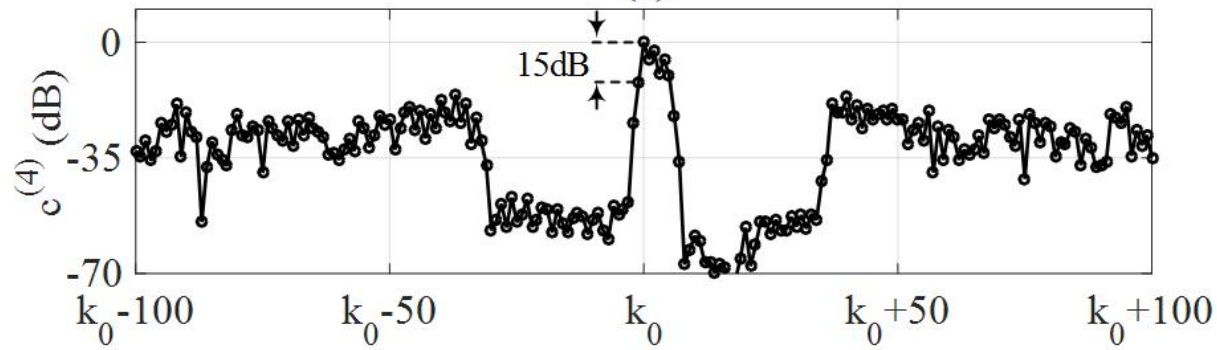

(d)

Figure 3.2: Autocorrelation level corresponding to the over-sampled signal with $M=4$ and fine-resolution $k_{\tau_{0}}=3$.

represented by:

$$
\hat{\tau}_{0}=\left(M \hat{k}_{m^{*}}+m^{*}\right) \tilde{T}_{s}
$$

where $m^{*}$ and $\hat{k}_{m^{*}}$ denote the correlation path number containing the maximum rasing level and its corresponding index of maximum correlation level, respectively, 
achievable via (3.11).

The performance of the proposed technique is evaluated by comparing the mean square error (MSE) of the estimated ToA over $K$ independent run via:

$$
M S E_{\hat{\tau}}=\frac{1}{K} \sum_{k=1}^{K}\left|\tau_{0}^{(k)}-\hat{\tau}_{0}^{(k)}\right|^{2}
$$

where $\tau_{0}^{(k)}$ and $\hat{\tau}_{0}^{(k)}$ represent the $k^{\text {th }}$ ToA value and its estimation, respectively. Next section discusses designing a waveform with maximum rising level exploited in MRLD technique.

\subsubsection{Waveform Design}

\subsubsection{Forming the objective function}

The desired waveform sequence $\left\{s_{n}\right\}_{n=1}^{N}=\left\{e^{j \varphi_{n}}\right\}_{n=1}^{N}$ can be acquired as the solution of the following optimization problem:

$$
\begin{aligned}
& \hat{\phi}=\underset{\phi}{\operatorname{argmin}} \sum_{n=k}^{N-1} w_{k}\left|y_{k}\right|^{2} \\
& \text { s.t. } y_{k}=\sum_{k=1}^{N-1} s_{n} s_{N+n-k}^{*}, \text { for: } \quad s_{n}=e^{j \varphi_{n}}
\end{aligned}
$$


where $\phi=\left[\varphi_{1}, \varphi_{2}, \ldots, \varphi_{N}\right]^{T}$, and $N$, and $y_{k}$ and $w_{k}$ are defined in (3.1) and (3.5), respectively. Considering the Parseval energy equality in time-frequency domain and defining the $2 N$ point discrete Fourier transform of weighted sidelobes $\left\{w_{n} y_{n}\right\}_{p=1}^{N-1}$ as $\left\{S_{p}\right\}_{p=1}^{2 N}$, it can be shown that [108, 109]:

$$
\begin{aligned}
& W I S L=\frac{1}{4 N} \sum_{p=1}^{2 N}\left[S_{p}-N\right]^{2}, \\
& \text { for, } \quad S_{p}=\frac{1}{\sqrt{2 N}} \sum_{n=-(N-1)}^{N-1} w_{k}^{2} y_{k} e^{-j \frac{2 \pi k p}{2 N}},
\end{aligned}
$$

where $N$, and $y_{k}$ and $w_{k}$ are defined in (3.1) and (3.5), respectively. Substituting $y_{k}$ with (3.4) leads to [108, 109]:

$$
\begin{aligned}
S_{p} & =\frac{1}{\sqrt{2 N}} \sum_{k=-(N-1)}^{N-1} \sum_{n=k}^{N-1} s_{n} s_{N+n-k}^{*} w_{k}^{2} e^{-j \frac{2 \pi k p}{2 N}} \\
& =\sum_{l=1}^{N} \sum_{k=1}^{N} e^{-j \varphi_{k}} e^{j \frac{\pi k p}{N}} \Gamma_{k, l} e^{j \varphi_{l}} e^{-j \frac{\pi l p}{N}}
\end{aligned}
$$

where (.)* indicates the complex conjugate notation and $\Gamma_{k, l}$ represents the elements at the $k^{t h}$ row and $l^{\text {th }}$ column of squared weights matrix $\boldsymbol{\Gamma}$, defined such that:

$$
\boldsymbol{\Gamma}=\left[\begin{array}{cccc}
1 & \gamma_{1} & \ldots & \gamma_{N-1} \\
\gamma_{1} & 1 & \ldots & \gamma_{N-2} \\
\vdots & \vdots & \ddots & \vdots \\
\gamma_{N-1} & \gamma_{N-2} & \ldots & 1
\end{array}\right]
$$


This leads to the final format of waveform design objective function as follow:

$$
\begin{aligned}
& \hat{\phi}=\underset{\phi}{\operatorname{argmin}}\left\{\sum_{p=1}^{2 N}\left[S_{p}-N\right]^{2}\right\} \\
& \text { for, } \quad S_{p}=\sum_{l=1}^{N} \sum_{k=1}^{N} e^{-j \varphi_{k}} e^{j \frac{\pi k p}{N}} \Gamma_{k, l} e^{j \varphi_{l}} e^{-j \frac{\pi l p}{N}}
\end{aligned}
$$

where $N$, and $y_{k}$ and $w_{k}$ are defined in (3.1) and (3.5), respectively, and $\Gamma_{k, l}$ represents the element at the $k^{\text {th }}$ row and $l^{\text {th }}$ column of $\boldsymbol{\Gamma}$ defined in (3.17). Next section introduces a novel optimization approach that exploits the trust-region algorithm to optimize (3.18) via solving the system of nonlinear equations corresponding to $\nabla_{\phi} f=0$ for $f=\sum_{p=1}^{2 N}\left[S_{p}-N\right]^{2}$, where $S_{p}$ is defined in 3.18 .

\subsubsection{Trust-region Algorithm}

In order to find the optimum solution for the proposed objective function in (3.18), the trust-region algorithm is exploited to solve $\nabla_{\phi} f=0$ for $f=\sum_{p=1}^{2 N}\left[S_{p}-N\right]^{2}$, where $S_{p}$ is defined in $(3.18)$. Here, we define $\mathbf{g}=\nabla_{\phi} f$, and $\mathbf{H}=\nabla_{\phi}^{2} f$ as the gradient vector and Hessian matrix of the proposed objective function in (3.18). The analytical expressions for the gradient vector and Hessian matrix are discussed in Appendix A and B, respectively.

Algorithm 1 proposes the trust-region method to solve the system of nonlinear equations corresponding to $\nabla_{\phi} f=0$. Trust-region and Levenberg-Marquardt algorithms 
are the leading state-of-the-art approaches to solve a system of nonlinear and nonconvex equations (regarding $\mathbf{H}$ is negative definite). The trust-region technique aims to find the optimum step size $\mathbf{s}$ without exceeding the trust-region radius, $\delta$ which leads to quick convergence to a more interesting area [121], and therefore much better performance compare to Levenberg-Marquardt approach. This interesting property demands for finding the optimum step size at each iteration which is not a trivial problem regarding its quadratic form subject to one non-linear constraint (see Algorithm 1 line 4). However, the off-line procedure of waveform design justifies selecting the approach with higher computational complexity for better performance. Here, the Dogleg approach [121] is introduced to find the optimum step size at each iteration (see Algorithm 2). Next section discusses the impact of several parameters such as signal length and oversampling rate via simulations. It can be observed that the resolution of the proposed ToA estimation technique significantly improves by increasing the oversampling rate or equivalently the number of correlation paths and transmitted waveform length.

Figure 3.3 (a) and (b) depicts the autocorrelation levels of the designed waveforms exploiting Algorithm 1 for $N=128$ and $N=512$, respectively. Here, the exploited 


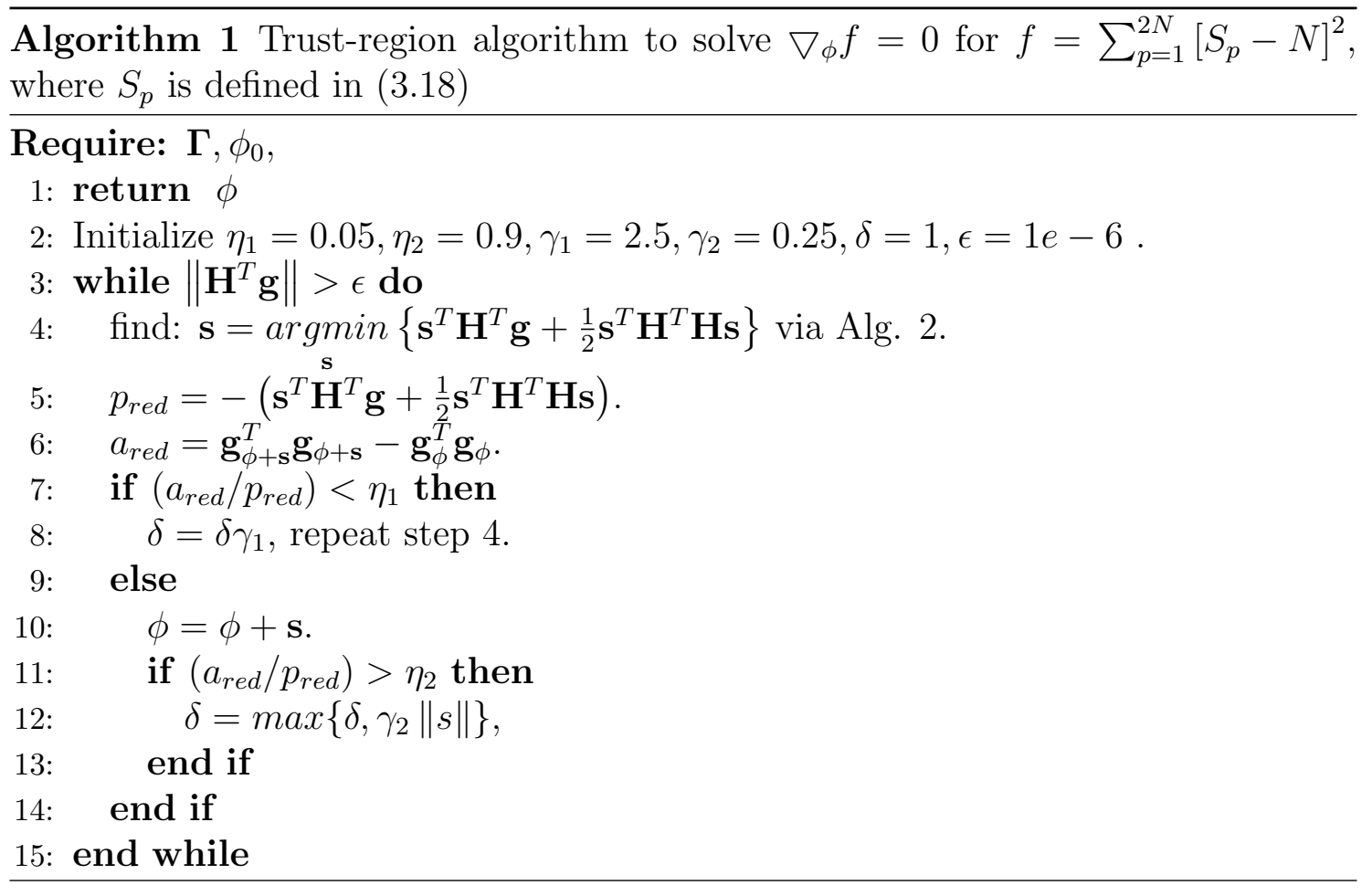

$\overline{\text { Algorithm } 2 \text { The Dogleg approach that find the optimum step size at each step }}$

\section{Require: $\mathbf{H}, \mathbf{g}, \delta$}

1: return $\mathbf{s}_{d l}$

2: $\mathbf{s}_{c}=-\delta \frac{\mathbf{H}^{T} \mathbf{g}}{\left\|\mathbf{H}^{T} \mathbf{g}\right\|}, \mathbf{s}_{n}=-\mathbf{H}^{-1} \mathbf{g}$.

3: $\mathbf{s}_{c}=\tau_{c} \mathbf{s}_{c}$ for: $\tau_{c}=\min \left\{1,\left\|\mathbf{H}^{T} \mathbf{g}\right\|^{3} /\left(\delta \mathbf{g}^{T} \mathbf{H}^{T} \mathbf{H g}\right)\right\}$

4: $a=\left\|\mathbf{s}_{c}-\mathbf{s}_{n}\right\|^{2}$.

$5: \quad b=0.5\left(\left\|\mathbf{s}_{c}\right\|^{2}+\left\|\mathbf{s}_{n}\right\|^{2}-a\right)$,

6: $\alpha=\frac{\left\|\mathbf{s}_{n}\right\|^{2}-\delta^{2}}{\left\|\mathbf{s}_{n}\right\|^{2}-b+\sqrt{b^{2}-\left\|\mathbf{s}_{c}\right\|^{2}\left\|\mathbf{s}_{n}\right\|^{2}+\delta^{2} a}}$,

7: $\mathbf{s}_{d l}=\alpha \mathbf{s}_{c}+(1-\alpha) \mathbf{s}_{n}$

squared weights are defined in (3.19) as follow:

$$
\gamma_{n}=\left\{\begin{array}{cc}
8 & n=N \\
0.6 & N-N_{o} \leq n \leq N-1 \\
0 & \text { elsewhere }
\end{array}\right.
$$



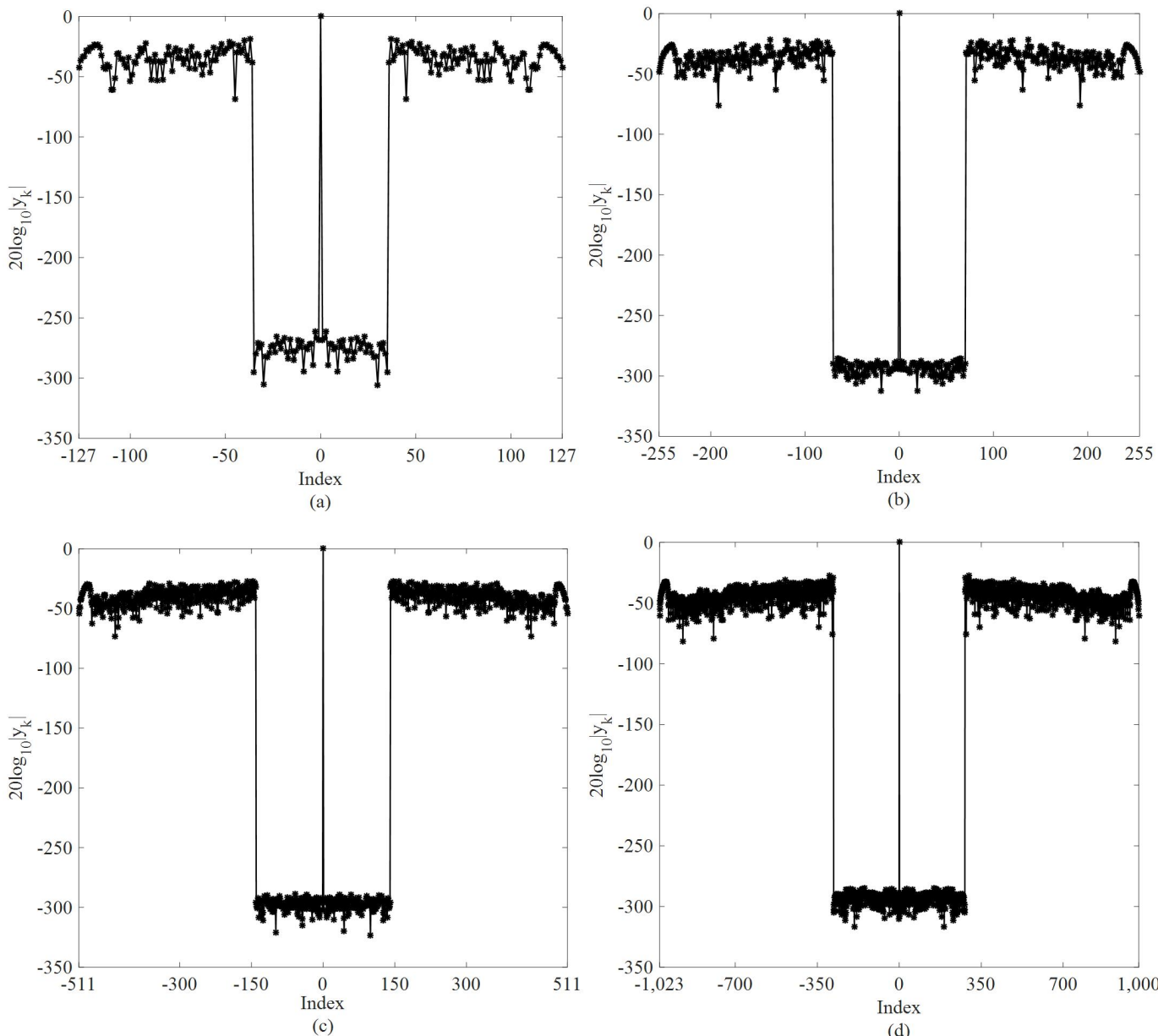

Figure 3.3: Autocorrelation level of designed waveform, (a) $N=128$, (b) $N=256$, (c) $N=512$, (d) $N=1024$.

where $N_{o}=35$ and 140 for Fig. 3.3 (a) and (b), respectively. Moreover, the initial phase values $\phi_{0}$ are chosen exploiting the Golomb sequence [108] which leads to $\varphi_{n}=\pi n(n-1) / N, \forall n=1,2, \ldots, N$, where $N$ denote the waveform length. 


\subsection{Simulation Results and Discussion}

Simulations are conducted to investigate the performance of the proposed ToA estimation method. Here, the mean square error (MSE) of the proposed ToA estimation in diverse scenarios is calculated and compared to SCAN [108] and ToA CRLB as benchmark. Moreover, the complexity of the proposed technique is investigated vs performance via discussing the impacts of the number of parallel paths or oversampling rate $M$, and waveform length $N$, on the total required operations and ToA MSE at different SNR regimes.

\subsubsection{Simulation Parameters and Methods}

In this sub-section, the details of simulated system model which is used for performance analysis are introduced. Table 3.1 shows the definitions and the values of parameters used to simulate the system model. In order to simulate the oversampled baseband signal at receiver as described in (3.8), the oversampled version of waveform is generated incorporating an ISI free pulse shaping filter such as raised cosine. The oversampled signal is passed through the flat and MPFS channels and added by independent samples of zero mean circularly complex white Gaussian noise. In 
Table 3.1

Simulation parameters and applied values

\begin{tabular}{|c|l|l|}
\hline Sym. & Definition & Value \\
\hline$T_{s}$ & Sampling time & $1 \mu s$ \\
$N$ & Length of waveform & $128,256,512,1024$ \\
$M$ & Number of correlation paths & $2 / 8 / 16 / 32 / 64 / 128$ \\
$L_{s}$ & Length of received signal & 5000 \\
$L$ & Taps of channel & $1 / 3 / 7$ \\
$T_{m}$ & Channel delay spread & $L \times T_{s}$ \\
$K$ & No. of independent runs & $10^{4}$ \\
\hline
\end{tabular}

order to verify the performance and stability of the proposed technique $K$ independent Monte-Carlo simulation is performed. Next sub-section details the simulation results.

\subsubsection{ToA Estimation Performance}

Figure 3.4 represents the MSE of estimated ToA exploiting the designed waveform in Section 3.3.2 for $N=512$, and the MRLD receiver in the presence of flat fading channel. Here, different numbers of correlation paths or oversampling rates are applied to investigate its impact on the proposed ToA estimator. The performance of proposed method is compared exploiting the waveform designed by SCAN proposed in [108], where $T_{s}=250 \mathrm{~ns}$ and the stop-band equals to $[-500 \mathrm{~K} \mathrm{~Hz}, 500 \mathrm{~K} \mathrm{~Hz}]$. Although the acquired attenuation for the frequencies outside the stop-band is around $30 \mathrm{~dB}$ which is insufficient for many applications, it can be observed that exploiting a band limited waveform with lower sampling time (higher resolution) offers stable 
results even at low SNR regimes. However, as shown in Fig. 3.4, applying the MRLD approach, higher resolution is achievable for medium to high SNR regimes $(\mathrm{SNR}>0 \mathrm{~dB})$ compared to SCAN.

Figure 3.5 represents the MSE of the estimated ToA exploiting the designed waveform in Section 3.3 .2 for $N=512$, and the MRLD receiver in the presence of unknown MPFS channels. Here, different numbers of correlation paths are applied to MRLD receiver to investigate its impact on the proposed ToA estimator and compare them with SCAN approach in a 7 taps MPFS channels. As shown in Fig. 3.5. the resolution of ToA estimator improves by increasing the number of correlation paths in the MRLD receiver. Moreover, it can be observed that the ToA MSE reaches to an error floor for high SNR values due to the deficiency of fine ToA estimation with higher resolution than $T_{s} / M$. Here, the amplitude and phase of CIR taps are selected independently exploiting Rayleigh and uniform distributions, respectively, where the largest amplitude is enforced to the first tap of CIR.

Figure 3.6 depicts the MSE of estimated ToA exploiting the designed waveform in Section 3.3 .2 for $N=512$, and the MRLD receiver in the presence of flat fading channel assuming no fine ToA is available, or $\tau_{0}=k \tilde{T}_{s}$. As shown in Fig. 3.6. the MSE of ToA increases exploiting higher number of correlation paths. This indicates that in the proposed MRLD receiver the probability of correct coarse ToA estimation decreases by increasing the correlation paths or oversampling rate, 
however, the overall performance of ToA estimation of MRLD receiver outperforms the traditional matched filter techniques exploiting full band or limited bandwidth waveforms such as SCAN regarding the error floor imposed by deficiency of fine ToA in these approaches.

\subsubsection{Complexity Analysis vs Performance}

Figure 3.7 represents the number of complex operations (summation and multiplication) required by the proposed technique vs different numbers of correlation paths $M$, and waveform length $N$. Considering $L_{r}$ as length of the received signal, $(N+2)\left(L_{r}-N\right)$ complex multiplications and $N\left(L_{r}-N\right)$ summations are required at each correlation path. As shown in Fig. 3.7, for a fix value of total required operations, multiple choices as a function of correlation path number or waveform length are available. Figure 3.8 depicts the MSE of the estimated ToA incorporating different numbers of waveform length and correlation paths. Here, it is aimed to reveal the impacts of regulating correlation path number or waveform length on the performance of the proposed method. As shown in Fig. 3.8, increasing the waveform length improves the MSE at low SNR values. For instance, the MSE represented by $N=1024, M=16$ is lower than the MSE proposed by $N=128,256, M=64$ for $-4 d B \leq S N R \leq 6 d B$. However, for higher SNR regimes increasing $M$ corresponds to lower MSE. Therefore, it can be observed that in low SNR regimes increasing 


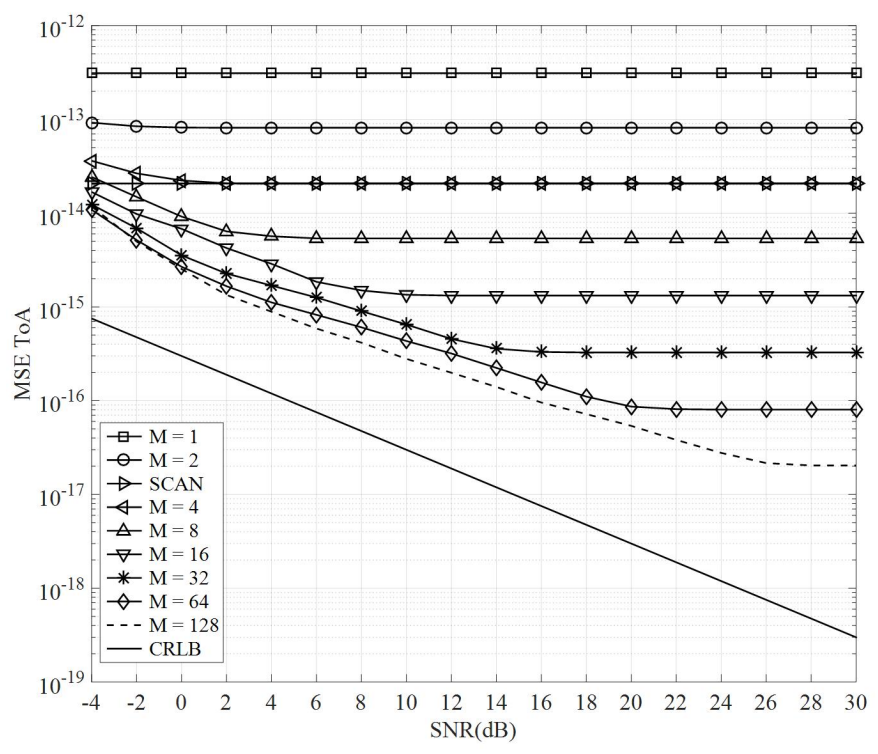

Figure 3.4: MSE of ToA exploiting different numbers of correlation paths $M$ vs SCAN with the same bandwidth $(1 \mathrm{MHz})$ in flat fading channel.

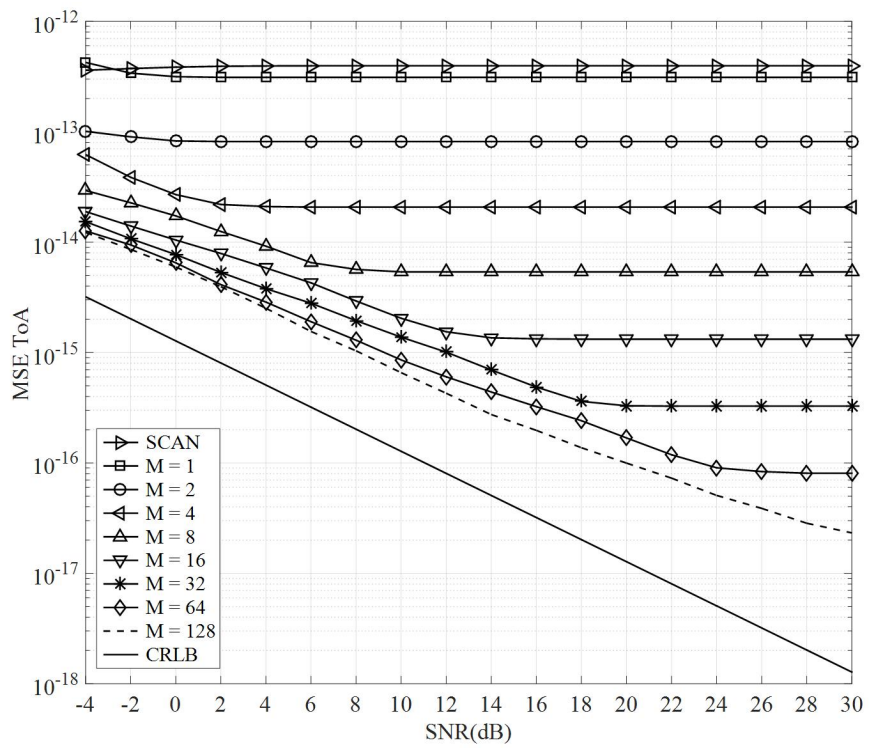

Figure 3.5: MSE of ToA exploiting different numbers of correlation paths $M$ vs SCAN with the same bandwidth $(1 \mathrm{MHz})$ in MPFS channels.

the waveform length $(N)$, is more beneficial; however, in higher SNR regimes expanding the number of correlation paths or oversampling rate $(M)$, is found more advantageous. 


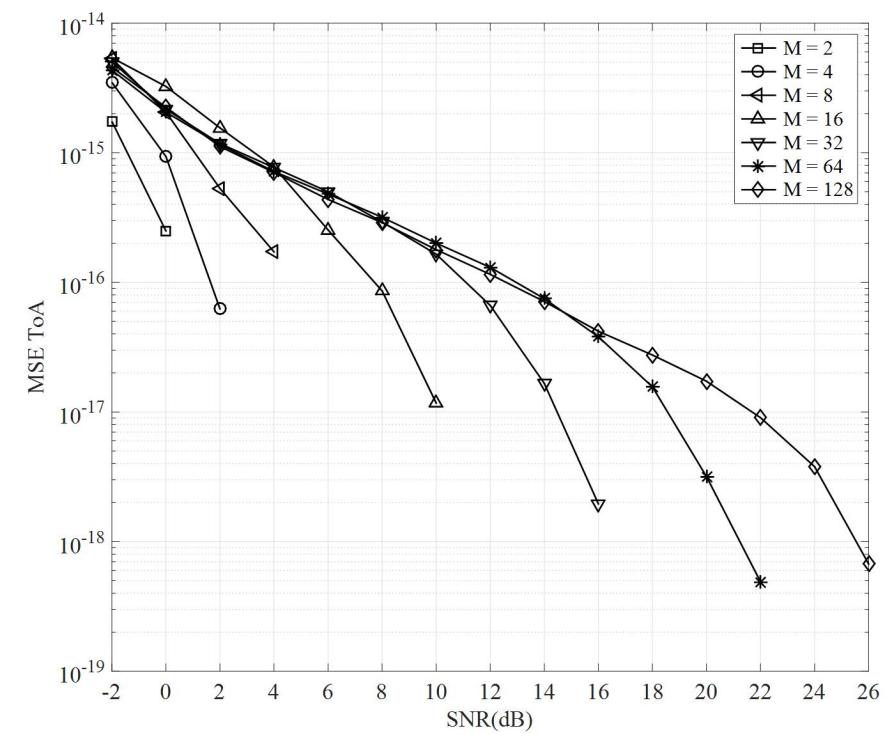

Figure 3.6: Impact of proposed MRLD technique on coarse ToA estimation.

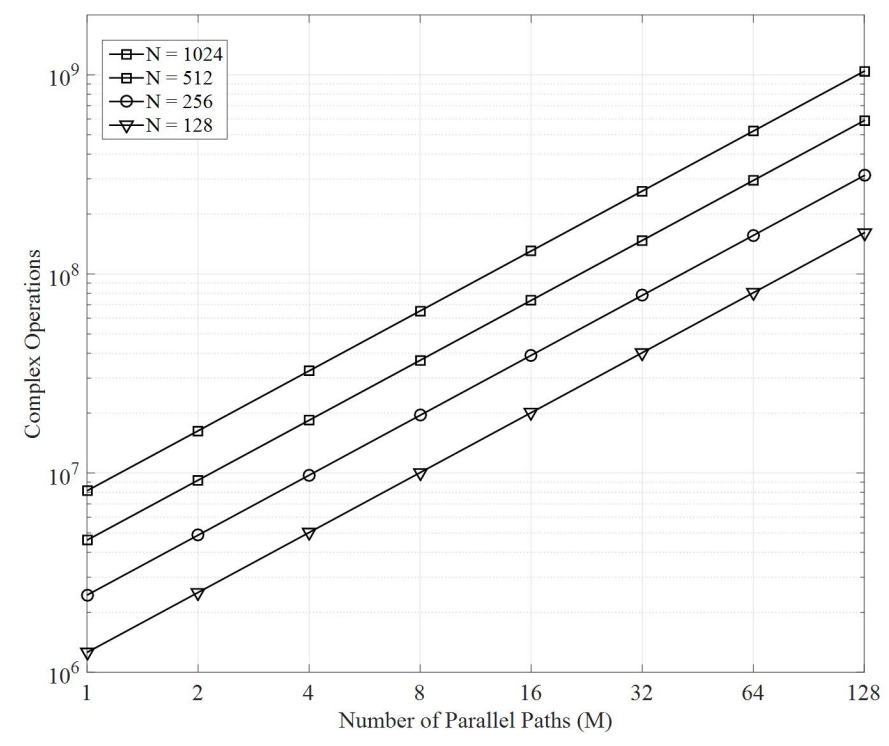

Figure 3.7: Total required complex operations by the proposed technique. 


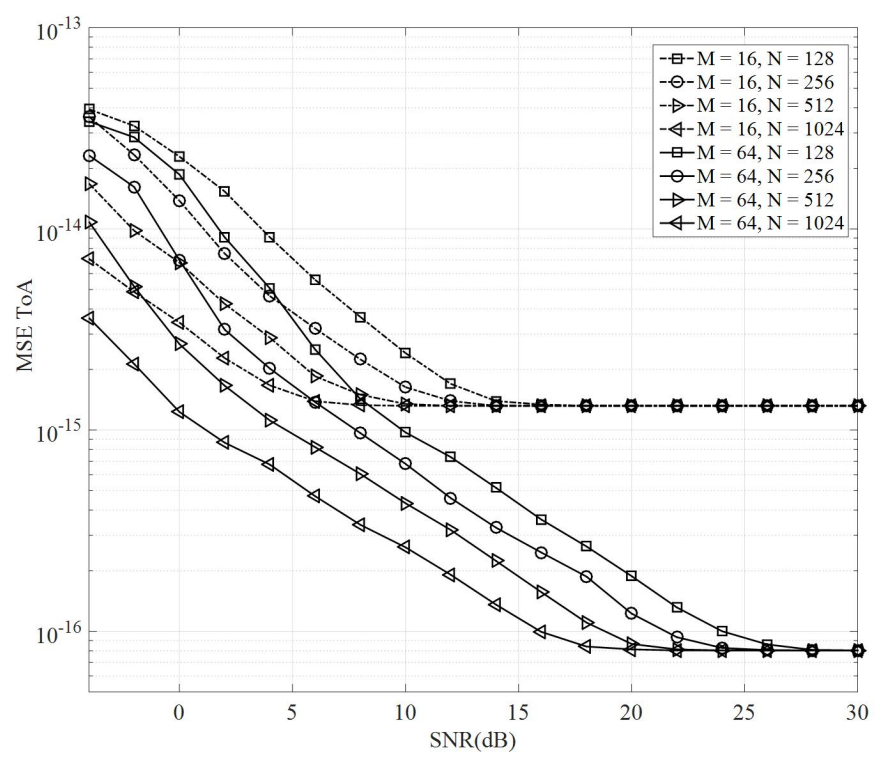

Figure 3.8: Impact of increasing the number of parallel paths $M$ vs waveform length $N$, on the performance of proposed technique.

\subsubsection{ToA CRLB}

The CRLB for the MSE of estimated ToA defined in (3.13) is proposed in Figures 3.4 and 3.5. Although approaching to the ToA CRLB is too optimistic specifically in low SNR regimes [122], it can be observed that increasing the correlation paths in MRLD receiver alleviates this gap at the cost of higher computational complexity and hardware cost. Considering $\mathbf{x}=\left[\mathbf{d}, h_{0}^{(I)}, h_{0}^{(Q)}, \ldots, h_{L-1}^{(I)}, h_{L-1}^{(Q)}\right]$ as unknown vector, where $\mathbf{d}=\left[\tau_{0}, \ldots, \tau_{L-1}\right]$, the log-likelihood function of probability distribution function of the received signal proposed in (3.8), $\mathbf{r}$, can be represented by:

$$
\Lambda_{\mathbf{r} \mid \mathbf{x}}=\Lambda_{0}-\frac{1}{\sigma^{2}}(\mathbf{r}-A \mathbf{h})^{H}(\mathbf{r}-A \mathbf{h}),
$$


The CRLB requires the computation of the Fisher information matrix (FIM), that corresponds to:

$$
J \triangleq E_{\mathbf{r} \mid \mathbf{x}}\left\{\nabla_{\mathbf{x}} \Lambda_{\mathbf{r} \mid \mathbf{x}} \cdot\left(\nabla_{\mathbf{x}} \Lambda_{\mathbf{r} \mid \mathbf{x}}\right)^{T}\right\}
$$

then, the CRLB for MSE of ToA can be represented by:

$$
C R L B_{\tau_{0}}=J_{i n v}(1,1)
$$

where $J_{i n v} \triangleq J^{-1}$. Appendix C details the computation of ToA CRLB. Applying $L=$ 1 and $h=1$, the CRLB of estimated ToA over AWGN channel can be represented by formerly discussed equation in [123, 124] as follow :

$$
C R L B_{\tau}=\frac{3 \sigma^{2}}{8 \pi^{2} N T_{s} \sigma_{s}^{2}\left(f_{2}^{3}-f_{1}^{3}\right)}
$$

where $N, T_{s}$ are defined in $(3.8)$, and $\sigma^{2}$ and $\sigma_{s}^{2}$ denote the variance of noise and transmitted waveform, respectively. Moreover, $f_{2}$ and $f_{1}$ represent the effective waveform's spectrum such that $B W=\left|f_{2}-f_{1}\right|$.

\subsection{Conclusion}

In this chapter, the MRLD technique is proposed which improves ToA resolution for a fixed available bandwidth in the presence of unknown multipath frequency selective 
(MPFS) channels. The proposed MRLD technique enjoys exploiting waveform with very high rising level at its correlation center and oversampling in several correlation paths at receiver to reveal higher resolution than sampling interval. Simulation results confirm that the resolution of estimated ToA can be improved via increasing the number of correlation paths in MRLD receiver, to a great extent in medium to high SNR regimes and increasing the waveform length at lower SNR values.

The very fact that MRLD technique exploits parallel processing to improve the ToA resolution makes this technique a proper candidate for entities recruiting multi-core processors such as cell phones or base-station receivers. Moreover, the significant desire on utilized bandwidth reduction in wireless technology endorses exploiting the MRLD receiver to increase ranging resolution and/or time synchronization while low bandwidth signal are exploited. Furthermore, applying the MRLD receiver improves the ToA resolution in the presence of frequency dispersive channel such as ionosphere layers and under-ground/water communications, where band limited waveforms must be applied to avoid signal dispersion. 


\section{Chapter 4}

\section{High Resolution ToA Estimation}

\section{in Non-homogenous, Frequency}

\section{Dispersive Channels ${ }^{1}$}

As discussed in Chapter 3 , incorporating wide band waveforms in free space is a feasible remedy to achieve high ToA resolution, however increasing the bandwidth of transmitted waveform in frequency dispersive channels intensifies frequency dispersion [70, 65] and [125]. In the microwave terminology, frequency dispersion refers

\footnotetext{
${ }^{1}$ The material contained in this chapter is submitted for publication in IEEE Transaction on Signal Processing, March, 2016
} 
to the principle that different frequency components of a transmitted electromagnetic waveform propagates with different velocities due to frequency dependency of medium's relative permeability and/or permittivity. Therefore, increasing transmitted waveform's bandwidth prompts higher frequency dispersion which alleviates the nonlinear distortion of transmitted waveform. The nonlinearity nature of the imposed distortion by frequency dispersion leads to a received signal that is barely detectable by the matched filter of transmitted waveform which dramatically degrades the ToA estimator performance [65].

To the best of authors knowledge, high resolution ToA estimation exploiting wideband microwave signals in NH media or single medium in frequency dispersion band is an open problem. Many researchers propose acoustics communication for ToA estimation in underwater or underground media [66]. Although acoustics communication offers high resolution for underwater ToA estimation, it cannot be applied to airborne to underwater channels due to strong reflections and attenuation in water/air boundary [67]. A few works [68]-[69], and [61]-64] propose ToA estimation in dispersive medium for specific scenarios which cannot be extended to NH media. In [68]-[69], time delays estimation of buried target echoes for GPR is addressed, meanwhile, the received echoes do not represent frequency dispersion assuming low conductivity and small layer thickness of sub-media. Although, these assumptions can be feasible for dry media, however, when the water content of media exceeds $10 \%$ by weight, the frequency dispersion must be considered due to the dielectric 
relaxation of water [70]. In [61], the transmitter location is estimated by combining the measured ToA data with the knowledge about shape and position of the medium which is not a feasible assumption in NH media. Authors in 63], propose sensor node localization via ToA measurements meanwhile the procedure of ToA estimation in soil as frequency dispersive medium is not discussed. In [64], ToA estimation of short range seismic signals in dispersive environments is addressed, however the system model does not represent the frequency dispersion due to low bandwidth of applied seismic signals.

In this chapter, high resolution ToA estimation in NH media consisting of diverse frequency dispersive sub-media is addressed. The proposed technique exploits preallocated OFDMA subcarriers to construct a wideband ranging signal. Here, we show that each frequency component of the propagated waveform is received with different time delays and phases which dramatically increases the number of unknowns in the received signal system model. Then, we propose a novel approach which reduces the number of unknowns by linear approximation of imposed delays and phases to the allocated OFDMA subcarriers in frequency domain. These justified approximations replaces the imposed delays with the delay corresponding to the waveform spectrum center as the desired unknown which is estimated exploiting maximum likelihood (ML) estimator. 
The feasibility of the applied approximations are evaluated via theory and simulations in diverse scenarios with different propagation distance and required ToA resolution. Here, human body, airborne to underwater and multi-layers underground channel with different water contents exploiting wideband microwave signals are considered as examples of NH media consisting of frequency dispersive sub-media. The proposed NH media are simulated according to the state-of-the-art channel and propagation models for body [126] 127]-128, sea water [125] and underground channel [129, 130, 131]. Simulation results prove the feasibility and efficiency of the proposed technique in terms of ToA resolution compared to the allocated bandwidth of the transmitted waveform.

The rest of chapter is organized as follow. Section 4.1 introduces the system model. The proposed algorithm for ToA measurements is presented in Section 4.2, Section 4.3 evaluates the feasibility of proposed technique in terms of applied approximations and simulation results. Finally Section 4.4 concludes this chapter. 


\subsection{System Model}

Exploiting $2 K$ OFDMA subcarriers, the baseband samples of the ranging waveform corresponds to [132]:

$$
s_{n}=\frac{1}{\sqrt{N}} \sum_{k=1}^{2 K} S_{k} e^{j 2 \pi m_{k} \Delta f n T_{s}}, n=1,2, \ldots, N
$$

where $N, \Delta f$ and $T_{s}$ represent the total number of available subcarriers or the length of waveform, OFDMA subcarrier spacing and sampling interval, respectively. Moreover, $S_{k}$ represents the $k^{\text {th }}$ frequency domain symbol known at both transmitter and receiver. In 4.1), $2 K$ symmetric subcarriers are allocated to construct the ranging waveform, where $m_{k}$ represents the frequency index of the $k^{\text {th }}$ subcarrier such that:

$$
m_{k}=\left\{\begin{array}{cc}
K, K-1, \ldots, 1 & \text { for } k=1,2, \ldots, K \\
N-K, \ldots, N-1 & \text { for } k=K+1, \ldots, 2 K
\end{array}\right.
$$

where $N$ is defined in (4.1) and $2 K$ denote the number of allocated subcarriers. Here, symmetric subcarriers are selected to construct a uniform waveform in frequency domain which enables linear approximation of imposed delay to each subcarrier (see Remark 1). To maintain orthogonality across subcarriers, subcarrier spacing must 
satisfy $\Delta f=1 /\left(N T_{s}\right)$ which leads to:

$$
\left\{s_{n}\right\}_{n=1}^{N}=\frac{1}{\sqrt{N}} \sum_{k=1}^{2 K} S_{k} e^{j \frac{2 \pi n m_{k}}{N}}
$$

where $N, \Delta f, T_{s}, S_{k}$, and $m_{k}$ and $K$ are defined in (4.1) and (4.2), respectively. The $N$ samples of baseband waveform represented in (4.3) are passed through the digital to analog converter (DAC), with sampling interval $T_{s}$ to shape the analog form of ranging waveform. The created baseband signal is up-converted to the carrier frequency $f_{c}$, and transmitted through the NH channel. The transmitted waveform by target node is propagated in $\mathrm{NH}$ channel consisting of several frequency dispersive sub-media. Considering $h(t, \omega)$ as time and frequency dependent of available media channel impulse response, i.e.,

$$
h(t, \omega)=\sum_{l=0}^{L-1} h_{l}(\omega) \delta\left(t-\tau_{l}(\omega)\right)
$$

where $h_{l}$ is the complex value of channel tap gain and $\delta(t)$ is the Dirac delta function, respectively. The received signal $r(t)$, is represented by the aggregation of the delayed version of frequency dispersed waveforms from $L$ propagation paths.

$$
r(t)=\sum_{l=0}^{L-1} \frac{1}{2\left|\omega_{K}\right|} \int_{-\omega_{K}}^{\omega_{K}} h_{l}(\omega) S(\omega) e^{j \omega\left(t-\tau_{l}(\omega)\right)} d \omega+v(t)
$$


where $S(\omega)$ is the Fourier transform of the ranging waveform $s(t)$, and $\tau_{l}(\omega)$ and $2\left|\omega_{K}\right|$ represent the time delay imposed by the $l^{\text {th }}$ path into the waveform component with frequency of $\omega$ and the bandwidth of $s(t)$, respectively. Assuming the flat attenuation over different frequency components of each channel tap which leads to $h_{l}(\omega) \approx h_{l}$. Applying 4.5 to the analog to digital converter (ADC) with sampling rate of $f_{s}=1 / T_{s}$, where $T_{s}$ is considered as sampling interval of the baseband signal leads to:

$$
r_{n}=\frac{1}{\sqrt{N}} \sum_{l=0}^{L-1} h_{l} \sum_{k=1}^{2 K} S_{k} e^{j \frac{2 \pi m_{k}\left(n T_{s}-\tau_{k, l}\right)}{N T_{s}}}+v_{n}
$$

where $N, T_{s}, S_{k}$, and $m_{k}$ and $K$ are defined in 4.1 and 4.2 , respectively. Moreover, $r_{n}$ and $v_{n}$ represent the $n^{\text {th }}$ sample of the base-band received signal and additive, white zero-mean Gaussian noise, respectively and $\tau_{k, l}$ is the delay corresponding to the $k^{\text {th }}$ frequency component propagated from the $l^{\text {th }}$ path. Based on the conventional definition of ToA, it is desired to estimate the delay corresponding to the first arrived component $(k=1)$ propagated from the shortest path $l=0$. This is equivalent to the value of $\tau_{K, 0}$. However, in the next section, a novel technique is proposed, which exploits frequency domain analysis to estimate $\tau_{0,0}$, that represents the delay corresponding to the spectrum center of the transmitted waveform $k=0$, propagated from the shortest path or equivalently, $l=0$. The rest of chapter, denotes the $\tau_{0,0}$ the desired ToA or briefly ToA. 


\subsection{Proposed ToA Estimation Technique}

This section presents the proposed ToA estimation technique considering $N_{r}$ samples of the received signal proposed in (4.6) are available at the receiver. Here, receiver selects $N_{r}$ such that the maximum possible range is covered that corresponds to:

$$
N_{r}=\frac{1}{c T_{s}} \sum_{m=1}^{M} d_{\max }^{(m)} \sqrt{\varepsilon_{r}^{(m)}}
$$

where $c, T_{s}$ and $M$ denote the universal speed of light, sampling interval and the number of available sub-media in NH medium. Moreover, $d_{\max }^{(m)}$ and $\varepsilon_{r}^{(m)}$ represent the maximum possible propagation distance and the relative permittivity of $m^{\text {th }}$ available sub-media, respectively. Considering frequency domain ToA estimation, one can estimate $\tau_{0,0}$ in 4.6 given: $0 \leq \tau_{0,0} \leq(N-1) T_{s}$, however for $\tau_{0,0}>N T_{s}$

any frequency domain approach estimates $\tilde{\tau}_{0,0}=\tau_{0,0}-t_{s}$, for $t_{s}=\left\lfloor\frac{\tau_{0,0}}{N T_{s}}\right\rfloor N T_{s}$ where the $\lfloor$.$\rfloor operand rounds to the nearest integers towards minus infinity. Therefore,$ prior to the discussed ToA estimation technique, we need to propose a time frame detection technique which estimates $t_{s}$ such that $0 \leq \tau_{0,0}-t_{s} \leq(N-1) T_{s}$, where $\tau_{0,0}$ represents the desired ToA. In order to address this issue, a novel and efficient time frame detection technique is proposed in Section 4.2.1 which estimates $t_{s}$ prior to the discussed ToA estimation technique proposed in Section 4.2.2. 


\subsubsection{Time Frame Detection Technique}

The procedure of ToA estimation is initiated by calculating the start time $\left(t_{s}\right)$, corresponding to the time frame including the desired ToA or $\tau_{0,0}$, such that $0 \leq \tau_{0,0}-t_{s} \leq(N-1) T_{s}$. Here, a time frame denotes the time interval of $N$ consecutive samples of the received signal in (4.6), with an arbitrary start. Considering $N_{r}$ samples of the received signal are available according to (4.6) and (4.7), the accumulated energy of the transmitted OFDMA subcarriers in frequency domain at the $i^{\text {th }}$ time frame can be written by:

$$
E_{i}=\sum_{k=1}^{2 K}\left|\mathbf{w}_{k}^{T} \mathbf{r}_{i}\right|, i=1,2, \ldots,\left\lfloor\frac{N_{r}}{N}\right\rfloor,
$$

for $\mathbf{w}_{k}=\left[e^{-j \frac{2 \pi m_{k}}{N}}, e^{-j \frac{2 \pi 2 m_{k}}{N}}, \ldots, e^{-j \frac{2 \pi N m_{k}}{N}}\right]^{T}$, where $N$ and $m_{k}$ are defined in 4.1) and (4.2), respectively. Moreover, $\mathbf{r}_{i}=\left[r_{(i-1) N+1}, r_{(i-1) N+2}, \ldots, r_{i N}\right]^{T}$ where $r_{n}$ represents the $n^{t h}$ sample of the received signal in time domain defined in 4.6). Algorithm 3 details the proposed time frame detection technique based on frequency domain energy calculation in (4.8). Starting from the first sample corresponding to the time origin $t=0$, receiver calculates the accumulated energy of all $\left\lfloor\frac{N_{r}}{N}\right\rfloor$ available time frames using (4.8), and selects two time frames with the largest accumulated energy values, $E_{i_{1}}$ and $E_{i_{2}}$, such that $E_{i_{1}}>E_{i_{2}}$. If $E_{i_{1}}$ is large enough, for instance $E_{i_{1}}>\gamma E_{i_{2}}$ for $\gamma=5$, the algorithm selects the desired time frame start as $t_{s}=$ 


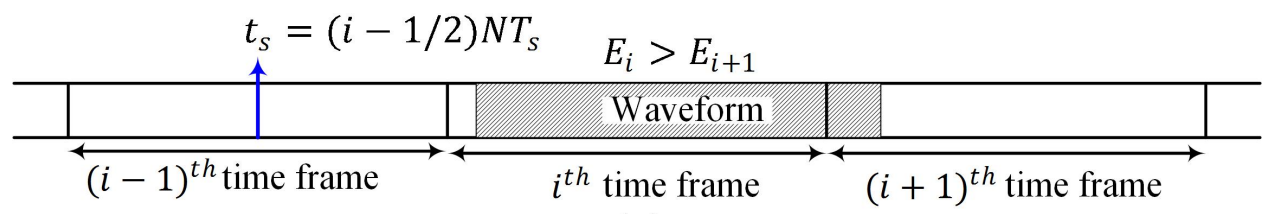

(a)

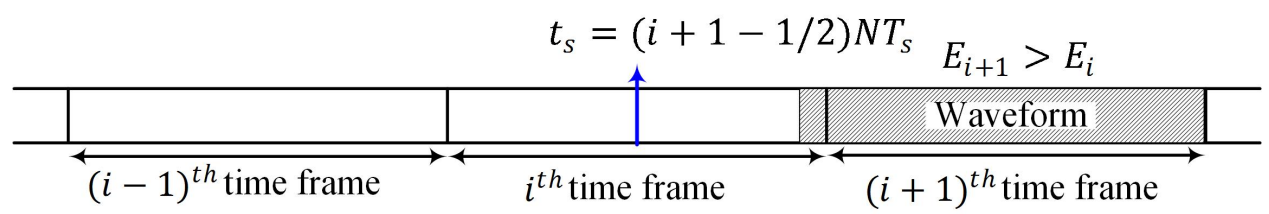

(b)

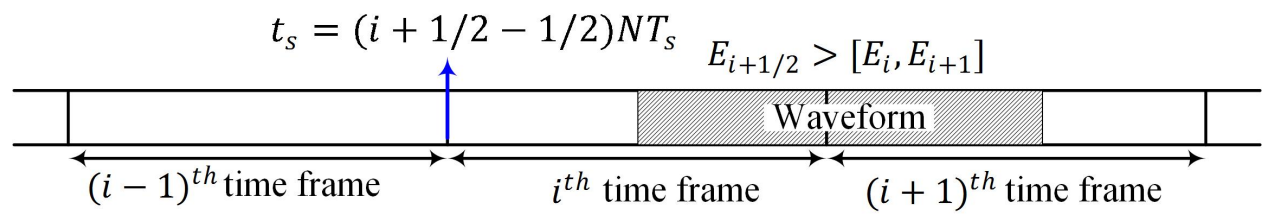

(c)

Figure 4.1: Three different scenarios of received waveform, (a) close to the time fame start, (b) close to the end of time frame, (c) in the middle of time frame.

$\left(i_{1}-1 / 2\right) N T_{s}$. This case occurs if the desired ToA is close enough to the start or the end of the $i^{t h}$ time frame $\left(\forall i=1,2, \ldots,\left\lfloor\frac{N_{r}}{N}\right\rfloor\right)$, as shown in Fig. 4.1(a) and (b). However, if the waveform arrival is close to the middle of the $i^{\text {th }}$ time frame as shown in Fig. 4.1(c), the accumulated energy of the $i^{\text {th }}$ and the $(i+1)^{t h}$ time frames are at the same order and can be manipulated with noise power. Therefore, if $E_{i_{1}}$ and $E_{i_{2}}$ do not satisfy $E_{i_{1}}>\gamma E_{i_{2}}$, the proposed algorithm selects a new time frame initiated by $i_{m}=\min \left\{i_{1}, i_{2}\right\}+1 / 2$, and computes its corresponding accumulated energy defined by $E_{i_{m}}$, using (4.8). At the final step, if $E_{i_{m}}>E_{i_{1}}$, the algorithm selects the desired time frame start from $t_{s}=\left(i_{m}-1 / 2\right) N T_{s}$. In the next section, the start time of the time frame containing the desired ToA $t_{s}$, is utilized for high 
resolution ToA estimation.

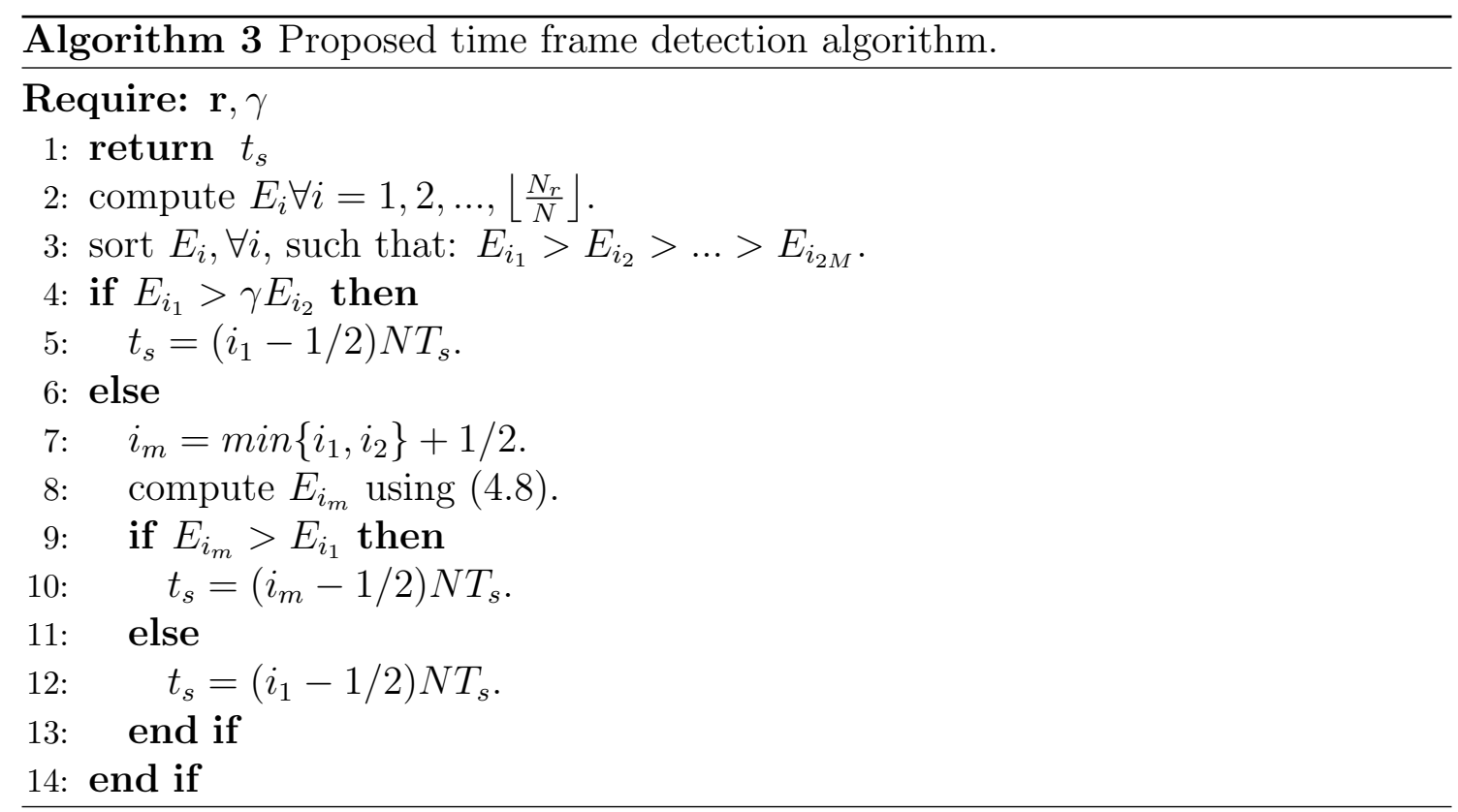

\subsubsection{High Resolution ToA Estimation}

Assuming the start time of the time frame containing the desired ToA $\left(t_{s}\right)$ is calculated using the proposed technique in Algorithm 3, receiver converts $3 N$ samples starting from $n_{s}=\frac{t_{s}}{T_{s}}$ into frequency domain applying discrete Fourier transform (DFT). Here, $2 N$ additional samples are selected to cover $N$ possible samples between the corresponding sample of the desired ToA and $n_{s}$, and $N$ samples for signal expansion due to frequency dispersion. Therefore, $2 K$ samples of the received signal 
in frequency domain are achievable via:

$$
R=\tilde{\mathbf{W}} \mathbf{r}_{n_{s}}
$$

where $\tilde{\mathbf{W}}=[\mathbf{W}, \mathbf{W}, \mathbf{W}]_{2 K \times 3 N}$, for $\mathbf{W}=\left[\mathbf{w}_{1}^{T} ; \mathbf{w}_{1}^{T} ; \ldots ; \mathbf{w}_{2 K}^{T}\right]_{2 K \times N}$ and $\mathbf{w}_{k}$ is a column vector defined in 4.8. . Moreover, $\mathbf{r}_{n_{s}}$ represents $3 N$ samples of received signal defined by $\mathbf{r}_{n_{s}}=\left[r_{n_{s}}, r_{n_{s}+1}, \ldots, r_{n_{s}+3 N-1}\right]^{T}$. Considering 4.6 as the time domain system model for the received signal, the system model for $k^{\text {th }}$ element of the received signal at frequency domain achievable via 4.9 can be written as:

$$
R_{k}=\frac{1}{N} \sum_{n=0}^{N-1} \sum_{l=0}^{L-1} h_{l} \sum_{k^{\prime}=1}^{2 K} S_{k^{\prime}} e^{j \frac{2 \pi m_{k^{\prime}}\left(n T_{s}-\tilde{\tau}_{l, k^{\prime}}\right)}{N T_{s}}} e^{-j \frac{2 \pi n m_{k}}{N}}+V_{k}
$$

where $N, T_{s}, S_{k}$, and $m_{k}$ and $K$ are defined in 4.1 and 4.2, and $V_{k}$ represents the $k^{t h}$ sample of additive noise at frequency domain. Here, $\tilde{\tau}_{l, k}$ represents the normalized delay corresponding to the $k^{\text {th }}$ transmitted subcarrier received from the $l^{\text {th }}$ path, defined by $\tilde{\tau}_{l, k}=\tau_{l, k}-t_{s}$, where $\tau_{l, k}$ is defined in 4.6 and $t_{s}$ represents the start time of the time frame containing the desired ToA achievable via Algorithm 3. Considering the orthogonality of transmitted subcarriers and applying some mathematical manipulation, 4.10 can be written as:

$$
R_{k}=S_{k} \sum_{l=0}^{L-1} h_{l} e^{-j \frac{2 \pi m_{k} \tilde{l}_{l, k}}{N T_{s}}}+V_{k}
$$


where $N, T_{s}, S_{k}$, and $m_{k}$ and $K$ are defined in (4.1) and (4.2), and $\tilde{\tau}_{l, k}$ and $V_{k}$ are defined in 4.10). The proposed system model in 4.11) represents $2 M$ nonlinear equations corresponding to $2 M$ allocated subcarriers, each contains $L(2 M+1)$ unknowns ( $L$ and $2 M L$ correspond to $h_{l}$ and $\tilde{\tau}_{k, l}$, respectively for $k \in \kappa$ and $l=0,1, \ldots, L-1$ ). The very fact that solving a system of $2 M$ nonlinear equations for $L(2 M+1)$, ( $L \geq 1$ ) unknowns leads to infinitely many solutions (or no feasible solution), demands for reducing the number of unknowns. In order to reduce the complexity of the proposed system model in (4.11), here, the case of flat fading channel is consider where the reflected copies of transmitted waveforms are small compared to the shortest path. Meanwhile, challenges and limitations of the proposed high resolution ToA estimation technique are discussed at the end of this section in the case of multipath, frequency dispersive channels. Therefore, applying $L=1$ in (4.11) leads to:

$$
R_{k}=S_{k} h e^{-j \frac{2 \pi m_{k} \tilde{\tau}_{k}}{N T_{s}}}+V_{k}
$$

where $h, \tilde{\tau}_{k}$ represent the attenuation coefficient and the propagation delay corresponding to the $k^{\text {th }}$ frequency component of transmitted waveform from the shortest path, respectively. Considering 4.12 as the system model for the received samples in frequency domain and known transmitted OFDMA symbols $\left(S_{k}\right)$, receiver calculates $X_{k}=Y_{k} / Y_{k+K}$ for $Y_{k}=R_{k} / S_{k}$ which leads to:

$$
X_{k}=\frac{e^{-j \frac{2 \pi m_{k} \tilde{\tau}_{k}}{N T_{s}}}}{e^{-j \frac{2 \pi m_{k+K} \tilde{\tau}_{k+K}}{N T_{s}}}}+\tilde{V}_{k}, \forall k=1,2, \ldots, K
$$


where $N, T_{s}$ and $\tilde{\tau}_{k}$ are defined in 4.1 and 4.12, respectively. Moreover, $\tilde{V}_{k}$ represents the additive measurement noise defined by $\tilde{V}_{k}=V_{k} / e^{-j \frac{2 \pi m_{k+K} \tilde{\tau}_{k+K}}{N T_{s}}}$. Substituting $m_{k+K}$ by $N-m_{k}$ according to (4.2), and applying some mathematical manipulations, 4.13 can be written as:

$$
X_{k}=e^{-j \frac{2 \pi m_{k}\left(\tilde{\tau}_{k}+\tilde{\tau}_{k+K}\right)}{N T_{s}}} e^{j \frac{2 \pi \tilde{\tau}_{k+K}}{T_{s}}}+\tilde{V}_{k}
$$

where $N, T_{s}, \tilde{\tau}_{k}$ and $\tilde{V}_{k}$ are defined in 4.1), 4.12 and 4.13), respectively. Here, a set of $K$ independent equations including $2 K$ unknowns are available which leads to infinitely many solutions (if available). However, it can be shown that (see the following Remark) the imposed delays to transmitted subcarriers can be approximated as a line.

Remark 1 Given any NH media consisting of $M$ sub-media satisfying:

$$
\begin{aligned}
\frac{1}{c} \mid \sum_{m=1}^{M} d^{(m)} \sqrt{\varepsilon_{r, k}^{(m)}}-\sum_{m=1}^{M} d^{(m)}\left[\left(\sqrt{\varepsilon_{r, K+1}^{(m)}}-\right.\right. \\
\left.\left.\sqrt{\varepsilon_{r, K}^{(m)}}\right) k+\sqrt{\varepsilon_{r, 1}^{(m)}}\right] \mid \leq \eta T_{s}, k=0, \ldots, 2 K-1,
\end{aligned}
$$

the imposed delay by frequency dispersion to the $k^{\text {th }}$ subcarrier $\left(\tau_{k}\right)$, can be approximated by $\hat{\tau}_{k}=a(k-1)+b$ such that $\left|\tau_{k}-\hat{\tau}_{k}\right| \leq \eta T_{s}, k=1, \ldots, 2 K$, where:

$$
a=\frac{1}{c} \sum_{m=1}^{M} d^{(m)}\left(\sqrt{\varepsilon_{r, K+1}^{(m)}}-\sqrt{\varepsilon_{r, K}^{(m)}}\right)
$$




$$
b=\frac{1}{c} \sum_{m=1}^{M} d^{(m)} \sqrt{\varepsilon_{r, 1}^{(m)}},
$$

and $c$ denotes the universal speed of light, and $T_{s}$ and $K$ are defined in (4.1) and 4.2), respectively and $\eta$ is and arbitrary small number $\eta<1$. Moreover, $\varepsilon_{r, k}^{(m)}$ and $d^{(m)}$ represent the relative permittivity of the $m^{\text {th }}$ media at the $k^{\text {th }}$ sub-carrier frequency, and its corresponding propagation distance, respectively.

Here, the slope of approximated line proposed in Remark 1 is selected according to the slope of the center of actual delays curve $\tau_{K+1}-\tau_{K}$, while the constant value is selected to converge the approximated line to the smallest delay $\tau_{1}$. More discussion in feasibility of the proposed approximation in Remark 1 is offered in section 4.3.2. Assuming all imposed delays $\left(\tau_{k}\right)$ are placed on approximated line proposed in Remark 1, the average of all equally spaced delays from line center, i.e. $\tau_{k}$ and $\tau_{k+K}$ are the same and equals to $\tau_{0}$ which is defined as the delay corresponding to the spectrum center of the transmitted waveform or the desired ToA. Therefore, $2 K$ unknown delays in 4.14 can be replaced by applying $\left(\tilde{\tau}_{k}+\tilde{\tau}_{k+K}\right)=2 \tilde{\tau}_{0}, \forall k=$ $1,2, \ldots, K$, which leads to:

$$
X_{k}=e^{-j \frac{4 \pi m_{k} \tilde{\tau}_{0}}{N T_{s}}} e^{j \frac{2 \pi \tilde{\tau}_{k+K}}{T_{s}}}+\tilde{V}_{k}
$$

where $N, T_{s}, \tilde{\tau}_{k}$ and $\tilde{V}_{k}$ are defined in 4.1, 4.12 and 4.13, respectively. Substituting $\tilde{\tau}_{k+K}$ with its corresponding coarse and fine delays such that $\tilde{\tau}_{k+K}=$ 
$\left(n_{k+K}+\delta_{k+K}\right) T_{s}$, gives:

$$
\begin{aligned}
X_{k} & =e^{-j \frac{4 \pi m_{k} \tilde{\tau}_{0}}{N T_{s}}} e^{j 2 \pi\left(n_{k+K}+\delta_{k+K}\right)}+\tilde{V}_{k} \\
& =e^{-j \frac{4 \pi m_{k} \tilde{\tau}_{0}}{N T_{s}}} e^{j 2 \pi \delta_{k+K}}+\tilde{V}_{k}, k=1,2, \ldots, K
\end{aligned}
$$

where $N, T_{s}$ are defined in (4.1), $\tilde{\tau}_{0}$ is defined in (4.17), and $\delta_{k}$ represents the fine delay corresponding to the $k^{\text {th }}$ transmitted subcarrier such that $0 \leq \delta_{k} \leq 1$. The proposed system model in (4.18) forms $K$ nonlinear and noisy equations containing one desired unknown $\tilde{\tau}_{0}$, and $K$ undesired unknowns $\delta_{k+K}$ for $k=1,2, \ldots, K$. Although the values of fine delays $\left(\delta_{k}\right)$ are negligible in terms of delay, ignoring them dramatically degrades the system model accuracy due to the impact of the exponential term, or $e^{j 2 \pi \delta_{k+K}}$. The following Remark proposes a linear approximation of the fine delays $\delta_{k+K}$ for $k=1,2, \ldots, K$ which reduces the number of unknowns proposed in (4.18). Here, an approximation in form of $a m_{k}+b$ (for any constant $a$ and $b$ ) is desired which satisfies acceptable error such that $\left|\delta_{k+K}-\hat{\delta}_{k+K}\right| \leq \eta T_{s}, k=1,2, \ldots, K$. Exploiting the form $a m_{k}+b$, the proposed system model in $(4.18)$ is converted to an exponential term corresponding to a single frequency which is simply detectable via ML estimator. 
Remark 2 Given any NH media consisting of $M$ sub-media satisfying:

$$
\begin{aligned}
& \mid \tau_{k+K}-\left\lfloor\frac{\tau_{k+K}}{T_{s}}\right\rfloor T_{s}+\frac{1}{c} \sum_{m=1}^{M} d^{(m)}\left(\sqrt{\varepsilon_{r, K+2}^{(m)}}-\right. \\
& \left.\sqrt{\varepsilon_{r, K+1}^{(m)}}\right)\left(m_{k}-1\right)-\delta_{2 K} \mid \leq \eta T_{s}, \forall k=1,2, \ldots, K
\end{aligned}
$$

given $\tau_{k+K}=\frac{1}{c} \sum_{m=1}^{M} d^{(m)} \sqrt{\varepsilon_{r, k+K}^{(m)}}$, the fine delay in 4.18 can be approximated linearly as $\hat{\delta}_{k+K} \cong-\beta^{\prime}\left(m_{k}-1\right)+\delta_{2 K}, \forall k=1,2, \ldots, K$, where

$$
\beta^{\prime}=\frac{1}{c} \sum_{m=1}^{M} d^{(m)}\left(\sqrt{\varepsilon_{r, K+2}^{(m)}}-\sqrt{\varepsilon_{r, K+1}^{(m)}}\right)
$$

and $T_{s}$, and $K$ and $m_{k}$ are defined in (4.1) and (4.2), respectively and $c, \eta, \varepsilon_{r, k}^{(m)}$ and $d^{(m)}$ are defined in (4.15).

Here, the slope of approximated line proposed in Remark 2 is selected according to the slope of the actual fine delay curve at the first point $\delta_{K+2}-\delta_{k+1}$, while the constant value is selected to converge the approximated line to the last value of actual fine delay curve $\delta_{2 K}$. More discussion in feasibility of the proposed fine delay approximation in Remark 2 is offered in section 4.3.2. According to Remark 2, the term $e^{j 2 \pi \delta_{k+K}}$ in 4.18 , can be approximated by $z_{0} e^{-j \frac{4 \pi m_{k}}{N} \beta}$, for $z_{0}=e^{j 2 \pi\left(\beta^{\prime}+\delta_{2 K}\right)}$ and $\beta=\frac{N \beta^{\prime}}{2}$. Substituting $z_{0} e^{-j \frac{4 \pi m_{k}}{N} \beta}$ into 4.18 , we have:

$$
X_{k}=z_{0} e^{-j \frac{4 \pi m_{k} \tilde{\tau}_{0}}{N T_{s}}} e^{-j \frac{4 \pi m_{k}}{N} \beta}+\tilde{V}_{k}=z_{0} e^{-j \frac{2 \pi m_{k}}{N}[2(\alpha+\beta)]}+\tilde{V}_{k}, \forall k=1,2, \ldots, K
$$


where $\alpha=\frac{\tilde{\tau}_{0}}{T_{s}}$, and $\beta$, denote two unknowns need to be estimated to reveal $\tilde{\tau}_{0}$. Defining $n^{(1)}=2(\alpha+\beta)$, it can be shown that (see Appendix A), the maximum likelihood estimator of $n^{(1)}$ can be written as:

$$
\hat{n}^{(1)}=\underset{n}{\operatorname{argmax}}\left|\mathbf{w}_{n}^{T} \mathbf{x}\right|,
$$

where $\mathbf{w}_{n}=\left[e^{j \frac{2 \pi K n}{N}}, e^{j \frac{2 \pi(K-1) n}{N}}, \ldots, e^{j \frac{2 \pi n}{N}}\right]^{T}$ is $K \times 1$ column vector and $\mathbf{x}=$ $\left[X_{1}, X_{2}, \ldots, X_{K}\right]^{T}$ for $X_{k}$ defined in 4.21. Exploiting 4.22, one can estimate $(\alpha+\beta)$, however, at least one linearly independent equation in terms of $\alpha$ and $\beta$ is required to estimate the desired ToA via $\tau_{0}=\tilde{\tau}_{0}+t_{s}$ where $\tilde{\tau}_{0}=\alpha T_{s}$. Here, we propose repeating the entire measurement procedure applying the same number of allocated subcarriers $(2 K)$ and waveform samples $(N)$, while, the sampling interval is changed, for instance to $T_{s}^{(p)}=p T_{s}$, where $T_{s}^{(p)}$ represents the sampling interval at the $p^{\text {th }}(p \geq 2)$ measurement.

Considering static channel where the propagation paths and hence their corresponding delays do not change during $P$ measurements, the delay corresponding to the spectrum center of the transmitted waveform $\tau_{0}$, is the same at each measurement. Applying $T_{s}^{(p)}=p T_{s}$, into 4.18 the frequency domain samples at the $p^{\text {th }}$ measurements can be written as:

$$
X_{k}^{(p)}=z_{0}^{(p)} e^{-j \frac{4 \pi m_{k} \tilde{\tau}_{0}}{N T_{s}^{(p)}}} e^{-j \frac{4 \pi m_{k}}{N} \beta^{(p)}}+\tilde{V}_{k}^{(p)}
$$


where $N$ and $\tilde{\tau}_{0}$ are defined in 4.1 and 4.17, respectively, and $T_{s}^{(p)}$ and $z_{0}^{(p)}$ are the applied sample time at transmitter and receiver, and the constant term $z_{0}=e^{j 2 \pi\left(\beta^{\prime}+\delta_{2 K}\right)}$, at the $p^{t h}$ measurement. Writing 4.20 for $\beta^{(p)}$ in terms of $T_{s}^{(p)}$ and substituting $T_{s}^{(p)}=p T_{s}$ leads to $\beta^{(p)}=\beta / p$. Applying $T_{s}^{(p)}=p T_{s}$ and $\beta^{(p)}=\beta / p$ 4.24 leads to:

$$
\begin{aligned}
X_{k}^{(p)} & =z_{0}^{(p)} e^{-j \frac{4 \pi m_{k} \tilde{\tau}_{0}}{N p T_{s}}} e^{-j \frac{4 \pi m_{k}}{N p} \beta}+\tilde{V}_{k}^{(p)}, \\
& =z_{0}^{(p)} e^{-j \frac{4 \pi m_{k}}{N p}\left(\alpha+\frac{\beta}{p}\right)}+\tilde{V}_{k}^{(p)}, \forall k=1,2, \ldots, K,
\end{aligned}
$$

where $N, K$ and $m_{k}$, and $\alpha, \beta$ are defined in (4.10) and (4.22), respectively. Therefore, considering $P$ independent measurements applying $T_{s}^{(p)}=p T_{s}$, for $p=1,2, \ldots, P$, and $\theta=[\alpha, \beta]^{T}$ leads to:

$$
\begin{gathered}
\theta=\left(\boldsymbol{\Gamma}^{T} \boldsymbol{\Gamma}\right)^{-1} \boldsymbol{\Gamma}^{T} \mathbf{n}, \\
\Gamma=\left[\begin{array}{llll}
1 & 1 / 2 & \ldots & 1 / P \\
1 & 1 / 4 & \ldots & 1 / P^{2}
\end{array}\right], \\
\mathbf{n}=\left[\frac{\hat{n}^{(1)}}{2}+n_{s}^{(1)}, \frac{\hat{n}^{(2)}}{2}+n_{s}^{(2)}, \ldots, \frac{\hat{n}^{(P)}}{2}+n_{s}^{(P)}\right]^{T},
\end{gathered}
$$

where:

$$
\hat{n}^{(p)}=\underset{n}{\operatorname{argmax}}\left|\mathbf{w}_{n}^{T} \mathbf{x}^{(p)}\right|
$$


and $\mathbf{w}_{n}^{T}$ is defined in 4.22. . Moreover, $n_{s}^{(p)}=t_{s}^{(p)} / T_{s}$ where $t_{s}^{(p)}$ and $T_{s}$ represent the start time of the time frame containing the desired ToA at the $p^{\text {th }}$ measurement achievable via Algorithm 3, and the sampling interval, respectively.

The performance of the proposed ToA estimation technique depends on the accuracy of the frequency domain detectors proposed in (4.26), which calculates the estimation of $2\left(\alpha+\frac{\beta}{p}\right)$. However, the returned value by 4.26 is time index and hence $0 \leq \hat{n}^{(p)} \leq N-1$ meanwhile, the value of $2(\alpha+\beta)$ can be a negative number or larger than $N$. In this case, the estimated value of $2\left(\alpha+\frac{\beta}{p}\right)$ via 4.26 would be different from its actual value. In order to resolve this issue, the estimated ToA via $\hat{\tau}_{0}=\alpha T_{s}$ needs to be inspected regarding its acceptable range $n_{s}^{(1)} T_{s} \leq \hat{\tau}_{0} \leq\left(n_{s}^{(1)}+N\right) T_{s}$ as follow. If $2(\alpha+\beta)>N$, the returned value by 4.26$)$ would be $2(\alpha+\beta)-N$. Applying this value into $4.25 \mathrm{c}$ shifts the estimated $\alpha$ by $N$ point to the left which leads to $\left(\alpha-n_{s}^{(1)}\right)<0$. Thus, if $\left(\alpha-n_{s}^{(1)}\right)<0,4.25 \mathrm{a}$ should be solved again using:

$$
\mathbf{n}=\left[\frac{\hat{n}^{(1)}+N}{2}+n_{s}^{(1)}, \frac{\hat{n}^{(2)}}{2}+n_{s}^{(2)}, \ldots, \frac{\hat{n}^{(P)}}{2}+n_{s}^{(P)}\right]^{T}
$$

where $n_{s}^{(p)}$ and $\hat{n}^{(p)}$ are defined in $4.25 \mathrm{~b}$ and 4.26 , respectively. If $2(\alpha+\beta)<0$, the returned value by 4.26 ) would be $N-2(\alpha+\beta)$. Applying this value into $4.25 \mathrm{c}$ shifts the estimated $\alpha$ by $N$ point to the right which leads to $\left(\alpha-n_{s}^{(1)}\right)>N$. Thus, 
if $\left(\alpha-n_{s}^{(1)}\right)>N, 4.25 \mathrm{a}$ should be solved again using:

$$
\mathbf{n}=\left[\frac{N-\hat{n}^{(1)}}{2}+n_{s}^{(1)}, \frac{\hat{n}^{(2)}}{2}+n_{s}^{(2)}, \ldots, \frac{\hat{n}^{(P)}}{2}+n_{s}^{(P)}\right]^{T}
$$

where $n_{s}^{(p)}$ and $\hat{n}^{(p)}$ are defined in 4.25b and 4.26, respectively. Algorithm 4 details the proposed high-resolution ToA estimation technique in NH medium considering $P$ ToA measurements. In the case of multipath channels, the system model

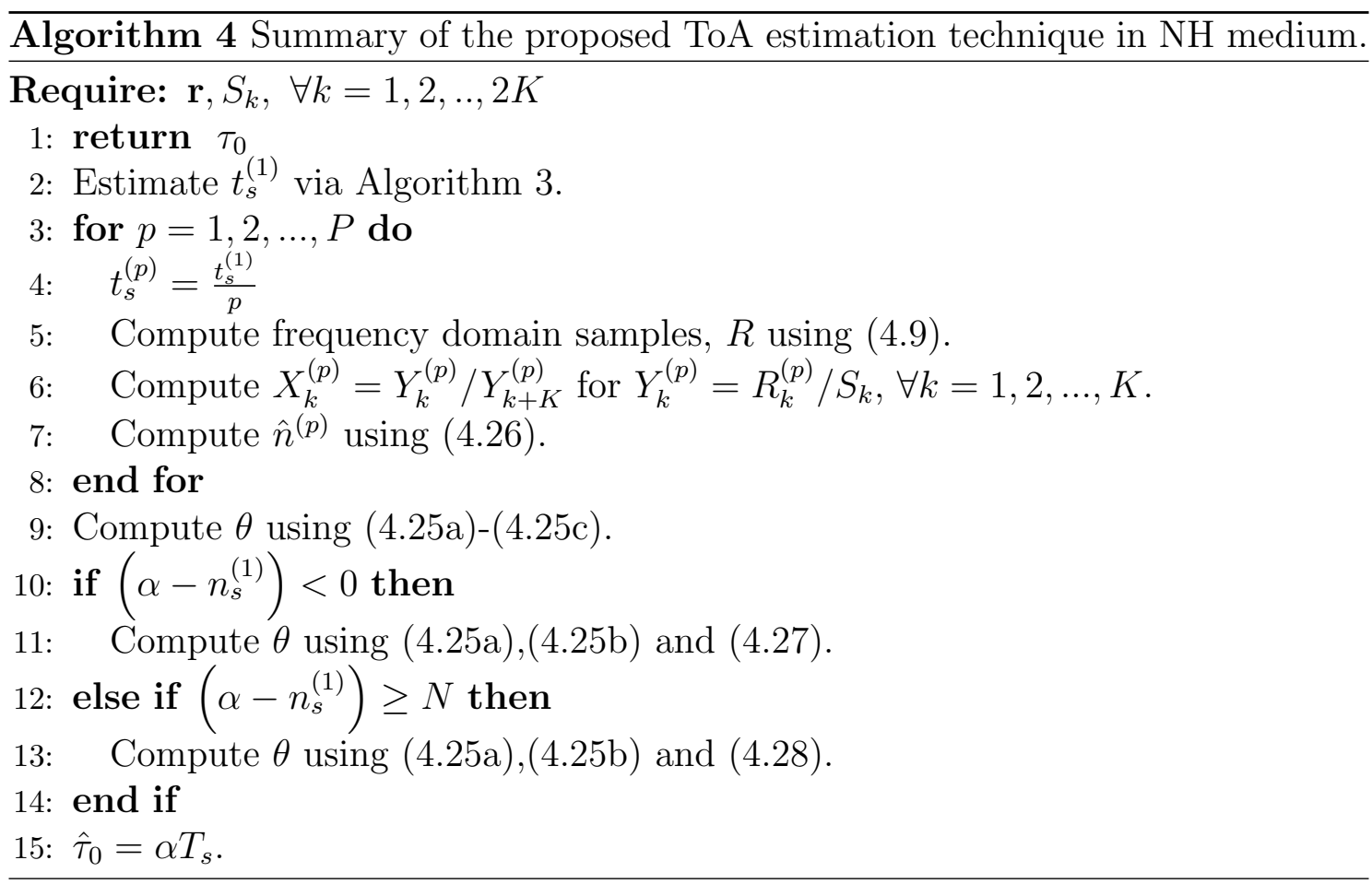

of received samples frequency domain proposed in 4.13 can be written as:

$$
\begin{aligned}
X_{k} & =\frac{h_{0} e^{-j \frac{2 \pi m_{k} \tilde{\tau}_{0, k}}{N T_{s}}}+\sum_{l=1}^{L-1} h_{l} e^{-j \frac{2 \pi m_{k} \tilde{\tau}_{l, k}}{N T_{s}}}}{h_{0} e^{-j \frac{2 \pi m_{k+K} \tilde{\tau}_{0, k+K}}{N T_{s}}}+\sum_{l=1}^{L-1} h_{l} e^{-j \frac{2 \pi m_{k+K} \tilde{\tau}_{l, k+K}}{N T_{s}}}}+\tilde{V}_{k}, \\
& =z_{1} e^{-j \frac{2 \pi m_{k}\left(\tilde{\tau}_{k}+\tilde{\tau}_{k+K}\right)}{N T_{s}}} e^{j \frac{2 \pi \tilde{\tau}_{k+K}}{T_{s}}}+\tilde{V}_{k},
\end{aligned}
$$


for:

$$
z_{1}=\frac{1+\sum_{l=1}^{L-1} \frac{h_{l}}{h_{0}} e^{-j \frac{2 \pi m_{k}\left(\tilde{\tau}_{l, k}-\tilde{\tau}_{0, k}\right)}{N T_{s}}}}{1+\sum_{l=1}^{L-1} \frac{h_{l}}{h_{0}} e^{-j \frac{2 \pi m_{k+K}\left(\tilde{\tau}_{l, k+K}-\tilde{\tau}_{0, k+K}\right)}{N T_{s}}}},
$$

where $N, T_{s}, S_{k}, m_{k}, K$ and $\tilde{\tau}_{l, k}$ are defined in 4.10 and $\tilde{V}_{k}$ is the frequency domain noise of the $k^{t h}$ measurement. Applying the proposed approximation in Remarks 1 and 2, the system model for the frequency domain measurements in multipath channels proposed in 4.29 can be written as:

$$
X_{k}=\tilde{z}_{0} e^{-j \frac{2 \pi m_{k}}{N}[2(\alpha+\beta)]}+\tilde{V}_{k}, \forall k=1,2, \ldots, K,
$$

for $\tilde{z}_{0}=z_{0} z_{1}$, where $z_{0}$ and $z_{1}$ are unknowns complex numbers defined in (4.21) and 4.30, respectively. Comparing 4.29) and 4.21) it can be observed that the same ML estimator proposed in 4.22 , can be applied to estimate $(\alpha+\beta)$ in 4.29 . Therefore, we conclude that applying the same procedure proposed in Algorithm 4. one can estimate the ToA corresponding to the spectrum center $(k=0)$ of transmitted waveform propagated from the shortest path $(l=0)$, or $\tilde{\tau}_{0,0}$. The performance of the proposed ToA estimation exploiting Algorithms 3 and 4 are discussed in the next section considering human body and airborne to underwater as examples of frequency dispersive NH media. 


\subsection{Simulations Result and Discussions}

This section discusses the simulation method, parameters and results in three subsections. First, details of simulated media and transmitted waveform are introduced in sub-section 4.3.1. Then, the applied approximations proposed in Remark 1 and 2 are discussed in sub-section 4.3.2. Finally, simulations results for the proposed ToA estimation technique via Algorithms 3 and 4 are proposed in sub-section 4.3 .3 .

\subsubsection{Details of Simulated NH Media}

In this work, two different media are considered with different physical characteristics to evaluate the proposed ToA estimation in different scenarios. For the first media, a part of human body containing 5 sub-media including fat, muscle, liver, body fluid and stomach is considered according to relative permittivities proposed by [133]. Here, human body is selected as an example of multi-layer NH media, where very high resolution is required, while the overall propagation distance is small. For the second media, a combination of free space and sea water is selected where lower resolution compared to human body is required, however much larger propagation distance must be considered. 
Table 4.1

Simulation parameters and applied values

\begin{tabular}{|l|c|c|c|c|}
\hline Definition & Symbol & Human Body & Airborne-Water & Underground \\
\hline Sample time & $T_{s}$ & $0.1 \mathrm{~ns}$ & $1 \mathrm{~ns}$ & $1 \mathrm{~ns}$ \\
Length of waveform & $N$ & $2^{9}, 2^{10}, 2^{11}, 2^{12}$ & $2^{9}, 2^{10}, 2^{11}, 2^{12}$ & $2^{9}, 2^{10}, 2^{11}, 2^{12}$ \\
No. of sub-carriers & $K$ & $8,12,25,50$ & $8,12,25,50$ & $8,12,25,50$ \\
No. of measurements & $P$ & 2 & 2 & 2 \\
No. of sub-media & $M$ & 5 & 2 & 4 \\
No. of channel taps & $L$ & 5 & 1 & 1 \\
Carrier frequency & $f_{c}$ & $5 \mathrm{GHz}$ & $1 \mathrm{GHz}$ & $1 \mathrm{GHz}$ \\
Propagation distance & $d$ & $4-20 \mathrm{~cm}$ & $1-50 \mathrm{~m}$ & $10-25 \mathrm{~m}$ \\
\hline
\end{tabular}

Table 4.1 details the parameters definition, their corresponding symbols and simulation parameters for human body and sea water media. The last row in Table 4.1 presents the selected interval of propagation distance for both media. Table 4.2 describes the applied values of relative permittivities of sub-media in simulations vs the number of allocated sub-carriers according to [133] and [134. Here, $\varepsilon_{r, K}$ and $\varepsilon_{r, N-K}$ represents the permittivities corresponding to the highest ( $K^{\text {th }}$ subcarrier) and lowest $\left((N-K)^{t h}\right.$ subcarrier $)$ frequency components of transmitted waveform, respectively. The proposed permittivity values in Table 4.2 are obtained from [133] for human body organs and [125] for sea water exploiting the Debye model [135] for salt water, respectively. Here, human body is considered as multipath channels simulated according to the power delay profile and delay spread proposed in [126], [127] and [128. In [126] and [127, the parameters of the statistical description of inbody channel for two different depths 2 and $8 \mathrm{~cm}$, are proposed. Here, we have used the same model to simulate the amplitude of 5 multipath components of channel, however the corresponding phase of multipath components are selected randomly 
Table 4.2

Example of applied relative permittivities vs number of allocated sub-carriers (bandwidth) for $N=1024$.

\begin{tabular}{|c|c|c|c|c|c|c|c|c|}
\hline \multirow{2}{*}{ sub-media } & \multicolumn{2}{|c|}{8} & \multicolumn{2}{c|}{12} & \multicolumn{2}{c|}{25} & \multicolumn{2}{c|}{50} \\
\cline { 2 - 10 } & $\varepsilon_{r, K}$ & $\varepsilon_{r, N-K}$ & $\varepsilon_{r, K}$ & $\varepsilon_{r, N-K}$ & $\varepsilon_{r, K}$ & $\varepsilon_{r, N-K}$ & $\varepsilon_{r, K}$ & $\varepsilon_{r, N-K}$ \\
\hline sea water & 80.16 & 80.20 & 80.15 & 80.21 & 80.12 & 80.24 & 80.07 & 80.31 \\
\hline fat & 5.02 & 5.03 & 5.01 & 5.04 & 5.00 & 5.05 & 4.97 & 5.09 \\
\hline muscle & 49.43 & 49.64 & 49.33 & 49.74 & 49.12 & 49.95 & 48.70 & 50.35 \\
\hline liver & 39.14 & 39.37 & 39.03 & 39.48 & 38.81 & 39.71 & 38.36 & 40.16 \\
\hline body fluid & 65.71 & 65.90 & 65.61 & 65.99 & 65.41 & 66.17 & 65.02 & 66.52 \\
\hline stomach & 57.75 & 58.02 & 57.68 & 58.09 & 57.46 & 58.31 & 57.03 & 58.73 \\
\hline
\end{tabular}

considering uniform distribution in $[-\pi, \pi]$. The simulated delay spread of channel changes from $0.7 \mathrm{~ns}$ for $d=4 \mathrm{~cm}$, to $3.6 \mathrm{~ns}$ for $d=24 \mathrm{~cm}$ which are consistent with the measured delay spread reported by [126], [127] and [128].

Although, underwater channel modeling including pathloss measurements and/or multipath characterization are discussed for underwater acoustic [136] and optical communications [137], a few works [125, 67] and [138], propose channel modeling and path loss measurements of wideband microwave propagation in sea water. In [125] the propagation measurements exploiting waveform covering the whole band between $800 \mathrm{MHz}$ and $18 \mathrm{GHz}$ is proposed. It can be observed that the attenuation of propagated signal in salt water can be modeled via $e^{-\alpha(f) d}$, where $\alpha(f)$ and $d$ represent the attenuation coefficient as function of carrier frequency and propagation distance, respectively [125]. It can be observed that amplitude of wideband signals propagated in sea water attenuates with propagation distance, therefore for the underwater channel the line-off-sight path is considered since the amplitude of 


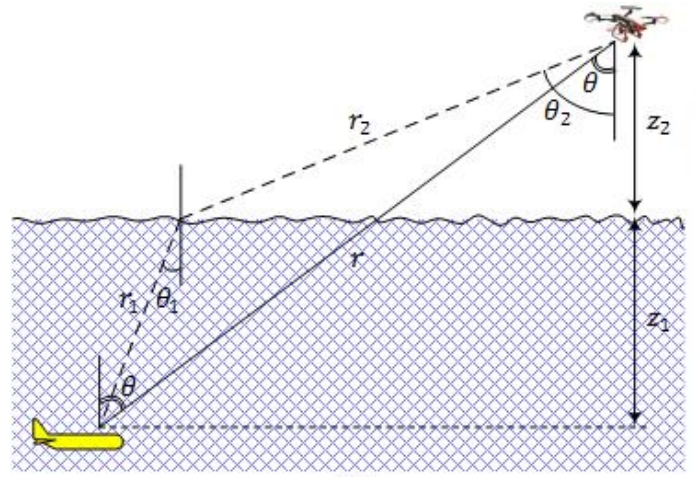

(a)

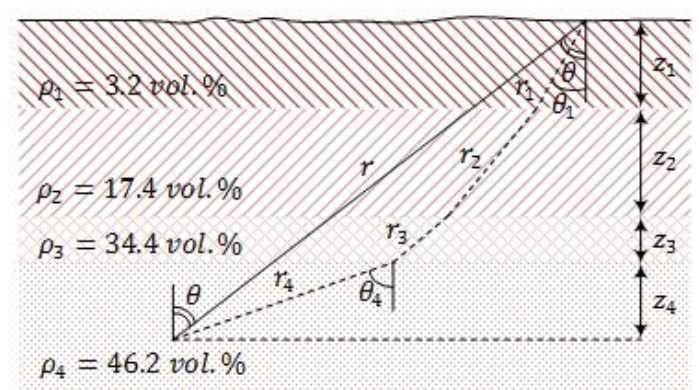

(b)

Figure 4.2: Applied NH media, (a) underwater-airborne, (b) four layers underground containing different water content in volume $\rho$.

reflection copies from sea bottom are negligible due to very high attenuation. Figure 4.2 (a) depicts airborne to shallow underwater as the first NH media. For the last media, a multi-layer underground channel with different water content $(\rho)$ at each layer is selected as shown in Fig. 4.2(b). Table 4.1 details the parameters definition, their corresponding symbols and simulations applied value for airborne to water and underground channels. The applied relative permittivities for sea water are simulated using the Debye model for salt water [125]. The applied values of relative permittivities for underground channel vs carrier frequency are proposed in Table 4.3, using soil dielectric spectra for different volumetric water contents in $20^{\circ} \mathrm{C}$ proposed in [129]. 
Table 4.3

Relative permittivities at different applied carrier frequencies $(\mathrm{GHz})$.

\begin{tabular}{|c|c|c|c|c|c|c|c|c|}
\hline$(\rho)$ & 0.1 & 0.4 & 0.6 & 1 & 2 & 4 & 6 & 10 \\
\hline $3.2 \%$ & 5.050 & 5.040 & 5.036 & 5.032 & 5.024 & 5.024 & 5.028 & 5.032 \\
\hline $17.4 \%$ & 15.346 & 13.211 & 12.019 & 10.590 & 10.385 & 10.385 & 10.590 & 9.000 \\
\hline $34.4 \%$ & 36.570 & 27.903 & 25.892 & 24.010 & 23.521 & 23.521 & 23.190 & 19.297 \\
\hline $46.2 \%$ & 48.806 & 35.417 & 33.178 & 30.192 & 28.705 & 27.896 & 26.157 & 21.662 \\
\hline
\end{tabular}

\subsubsection{Proposed Approximations Analysis}

In this section, the proposed approximations in Remarks 1 and 2 are evaluated via theory and simulating the actual and approximated values at two different scenarios applying practical parameters such as permittivity, sample time and propagation distance.

\subsubsection{Overall delays linearity}

The key point in Remark 1 is satisfying the approximation error inequality given by $\left|\tau_{k}-\hat{\tau}_{k}\right| \leq \eta T_{s}, k=1,2, \ldots, 2 K$ and small $\eta$. The proposed condition in (4.15) can be derived by substituting $\tau_{k}$ and $\hat{\tau}_{k}$ in $\left|\tau_{k}-\hat{\tau}_{k}\right| \leq \eta T_{s}$ by $\frac{1}{c} \sum_{m=1}^{M} d^{(m)} \sqrt{\varepsilon_{r, k}^{(m)}}$ and $a k+b$, respectively, for $a$ and $b$ are defined in (4.16). Therefore, it can be observed that if 4.15 holds for the given NH media, the proposed linear approximation in Remark 1, holds satisfying $\left|\tau_{k}-\hat{\tau}_{k}\right| \leq \eta T_{s}, k=1,2, \ldots, 2 K$. 

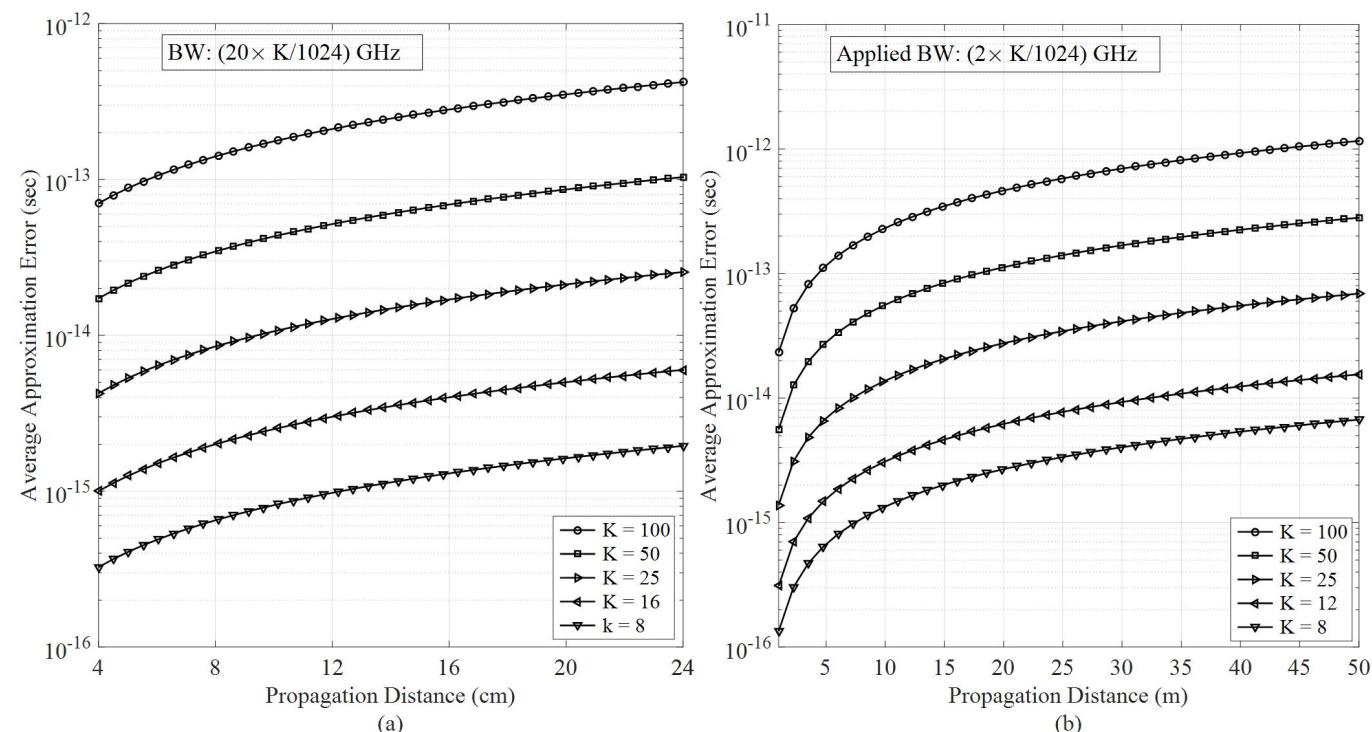

Figure 4.3: Average time error imposed by linear approximation proposed in Remark 1.

Although, the validity of the proposed condition in (4.15) cannot be evaluated at receiver as the values of propagation distances $\left(d^{(m)}\right)$ are unknown, however it can be evaluated for the worst case scenario where the maximum possible values for propagation distances are applied. Figure 4.3 depicts the average approximation error of subcarrier delays $\frac{1}{2 K} \sum_{k \in \kappa}\left|\tau_{k}-\hat{\tau}_{k}\right|$, for two scenarios (a) human body, (b) sea water, vs propagation distance, applying different allocated subcarriers. As shown, the average error increases by increasing the propagation distance and the number of allocated subcarriers or equivalently waveform bandwidth. However, given the worst case (largest propagation distance and waveform bandwidth), the approximation error is less than $1 \%$ and $0.4 \%$ of sampling time in human body and sea water, respectively. 


\subsubsection{Fine delay linearity}

The proposed approximation given the absolute value of approximation error in 4.19 is derived by subsisting $\delta_{k+K}$ and $\hat{\delta}_{k+K}$ in $\left|\delta_{k+K}-\hat{\delta}_{k+K}\right| \leq \eta T_{s}, k=1,2, \ldots, K$, by actual value of fine delay $\tau_{k+K}-\left\lfloor\frac{\tau_{k+K}}{T_{s}}\right\rfloor T_{s}$ and $-\beta^{\prime}\left(m_{k}-1\right)+\delta_{2 K}$ respectively, for $\beta^{\prime}$ proposed in 4.20). Although, similar to Remark 1, the absolute value of approximation error cannot be evaluated at receiver as the values of propagation distances $\left(d^{(m)}\right)$ are unknown, however it can be evaluated for the worst case scenario where the maximum possible values for propagation distances are applied. Figure 4.4 depicts the average of approximation error of fine delays

$\frac{1}{K} \sum_{k=1}^{K}\left|\delta_{k+K}-\left(-\beta^{\prime}\left(m_{k}-1\right)+\delta_{2 K}\right)\right|$, for two scenarios (a) human body and (b) sea water, vs propagation distance, applying different allocated subcarriers. As shown in Fig. 4.4, the average error increases by increasing the propagation distance and the number of allocated subcarriers or equivalently waveform bandwidth. However, given the worst case (largest propagation distance and waveform bandwidth), the approximation error is less less than $1 \%$ and $0.4 \%$ of sampling time human body and sea water, respectively. Therefore, it can be conclude that Remarks 1 and 2 are proposing valid approximations considering $0.01 \leq \eta<1$. 

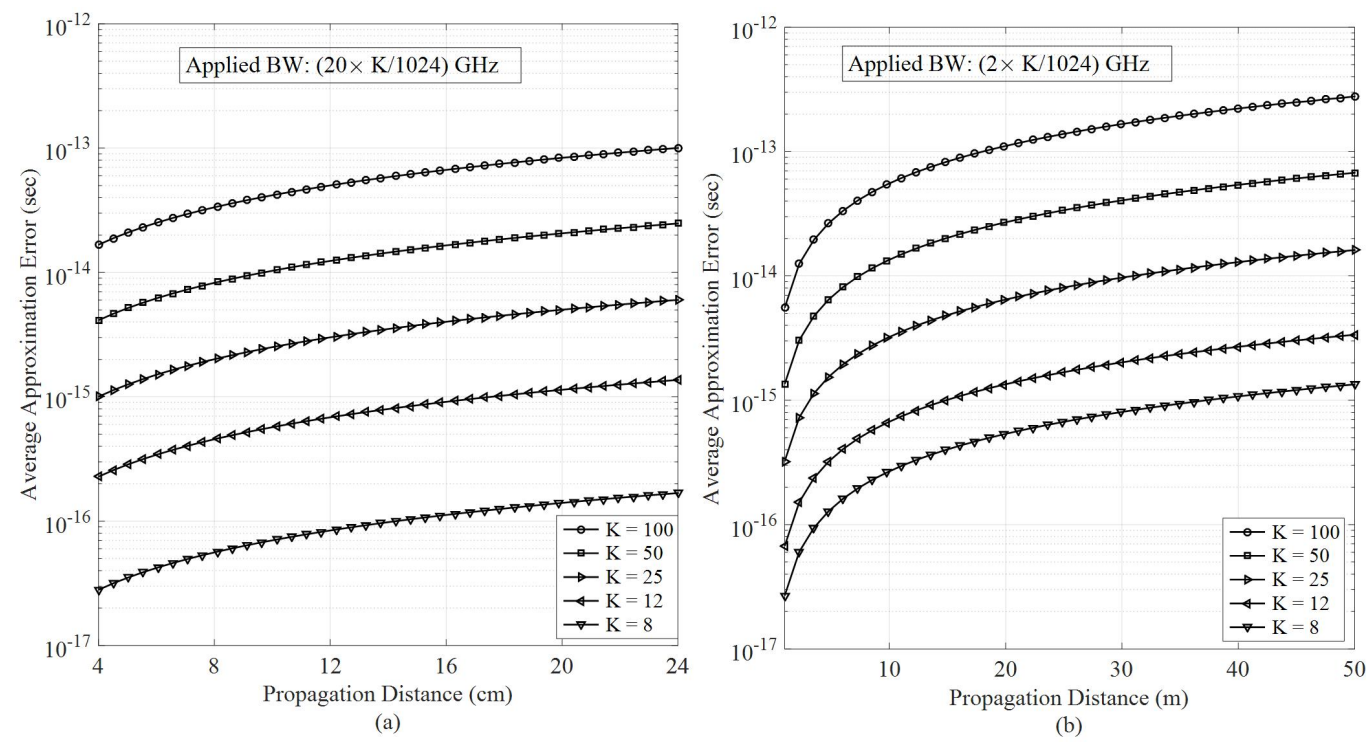

Figure 4.4: Average time error imposed by linear approximation proposed in Remark 2.

\subsubsection{Performance Analysis}

Figures 4.5(a)-(d) depict the average error of estimated ToA for human body. Details of simulation parameters and human body channel are discussed in Section 4.3.1. Here, human body is selected to represent a short range NH media which demands for very high ToA resolution. In Figs. 4.5(a)-(d) the average ToA error is evaluated vs SNR for $N=512,1024,2048$ and 4096, respectively, where $N$ denotes the length of the transmitted waveform. As shown in Figs. 4.5, increasing the number of allocated subcarriers improves the average ToA estimation error at medium to high SNR regimes (see Figs. 4.5 (a)-(d)). This improvement is acquired as allocating a larger number of subcarriers increases the frequency domain samples in (4.21). In the 

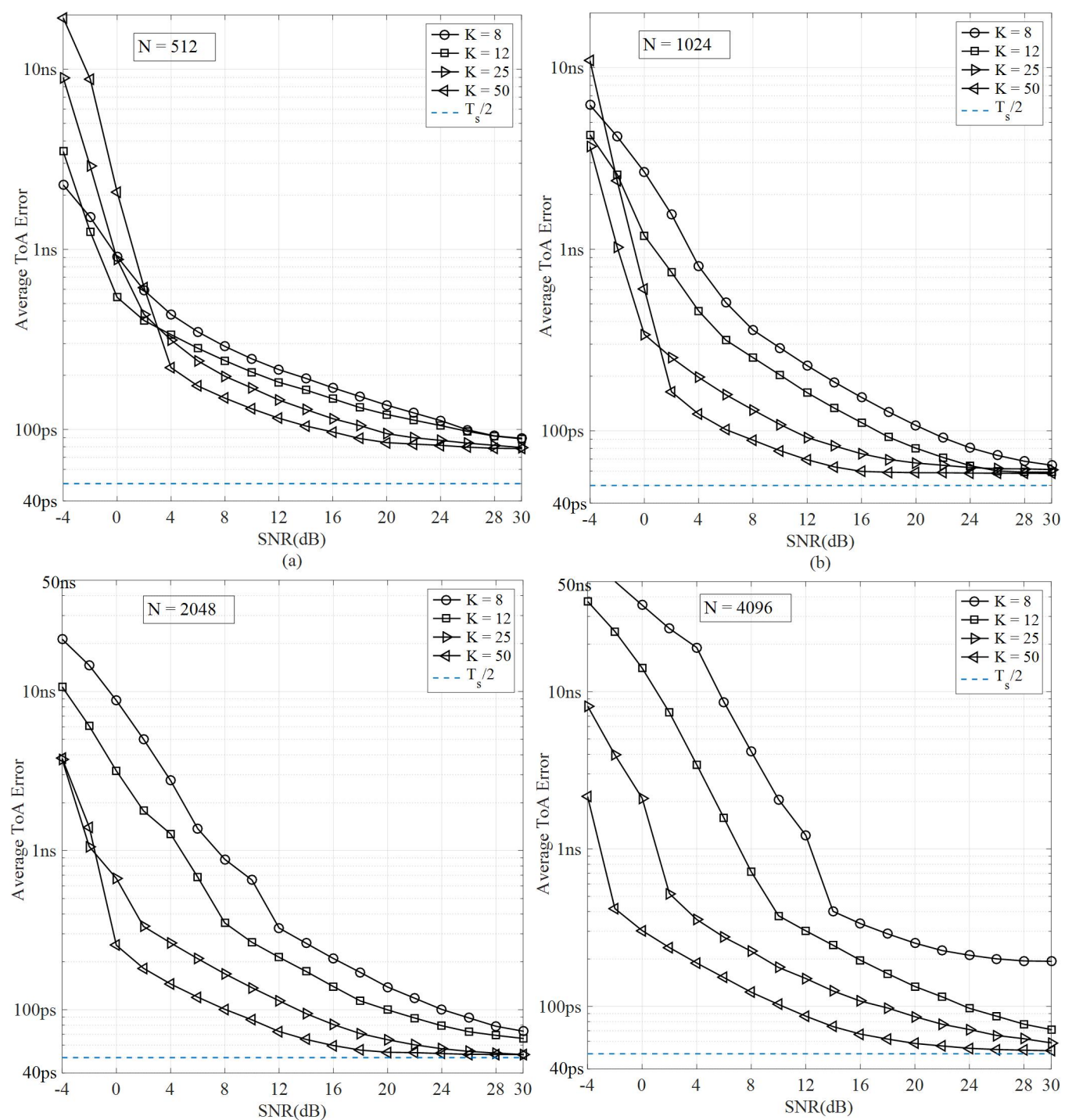

(c)

(d)

Figure 4.5: Impact of the number of allocated subcarriers and transmitted signal length on the estimated ToA average error in human body, (a) $N=$ 512, (b) $N=1024$, (c) $N=2048$, and (d) $N=4096$.

proposed technique, ToA is acquired from estimating the frequency of $X_{k}$ in (4.21) using 4.22 . Exploiting more samples improves the precision of interpolated signal, $X_{k}$, and hence, more accurate estimation can be proposed by 4.22). In Figs. 4.5(a) and (b) it can be observed that the average ToA estimation error increases at higher 
allocated subcarriers and low SNR regimes for shorter waveform length. Beside the positive impact of increasing the number of allocated subcarriers at medium to high SNR regimes, exploiting a larger number of subcarriers extends the in-band noise power which affects the ToA estimator performance at low SNR regimes specifically at higher sub-carrier spacing values (shorter signal length at fixed sampling time). This outcome can be observed in Figs. 4.5 (a) and (b) for $K=50$ and 25 for $-4 d B \leq S N R \leq 0$. Therefore, it can be concluded that the best ToA estimation is achievable via increasing the number of allocated subcarriers $K$, and the transmitted waveform length $N$, simultaneously as shown in Figs. 4.5 (c) and (d) for $K=25$ and 50. The same results can be observed for airborne to underwater scenario depicted in Figs. 4.6(a)-(d). The airborne to underwater channel is selected to represent a long range $\mathrm{NH}$ media with lower required ToA resolution compared to human body. Here, the same parameters and channel proposed in Section 4.3.1 are applied. As shown in Figs. 4.6(a)-(d), the proposed technique performs accurately at medium to high SNR regimes specifically for large number of allocated subcarriers and waveform length. Comparing Figures 4.5 and 4.6 it can be observed that the average ToA error converges to the $T_{s} / 2$ which is the minimum average error achievable via coarse ToA estimation.

Moreover, moving from Fig. 4.6(a) to Fig. 4.6(d), we observe that increasing the transmitted signal bandwidth improves ToA estimation error. This observation is consistent with our expectation that increasing bandwidth in a flat fading channel 

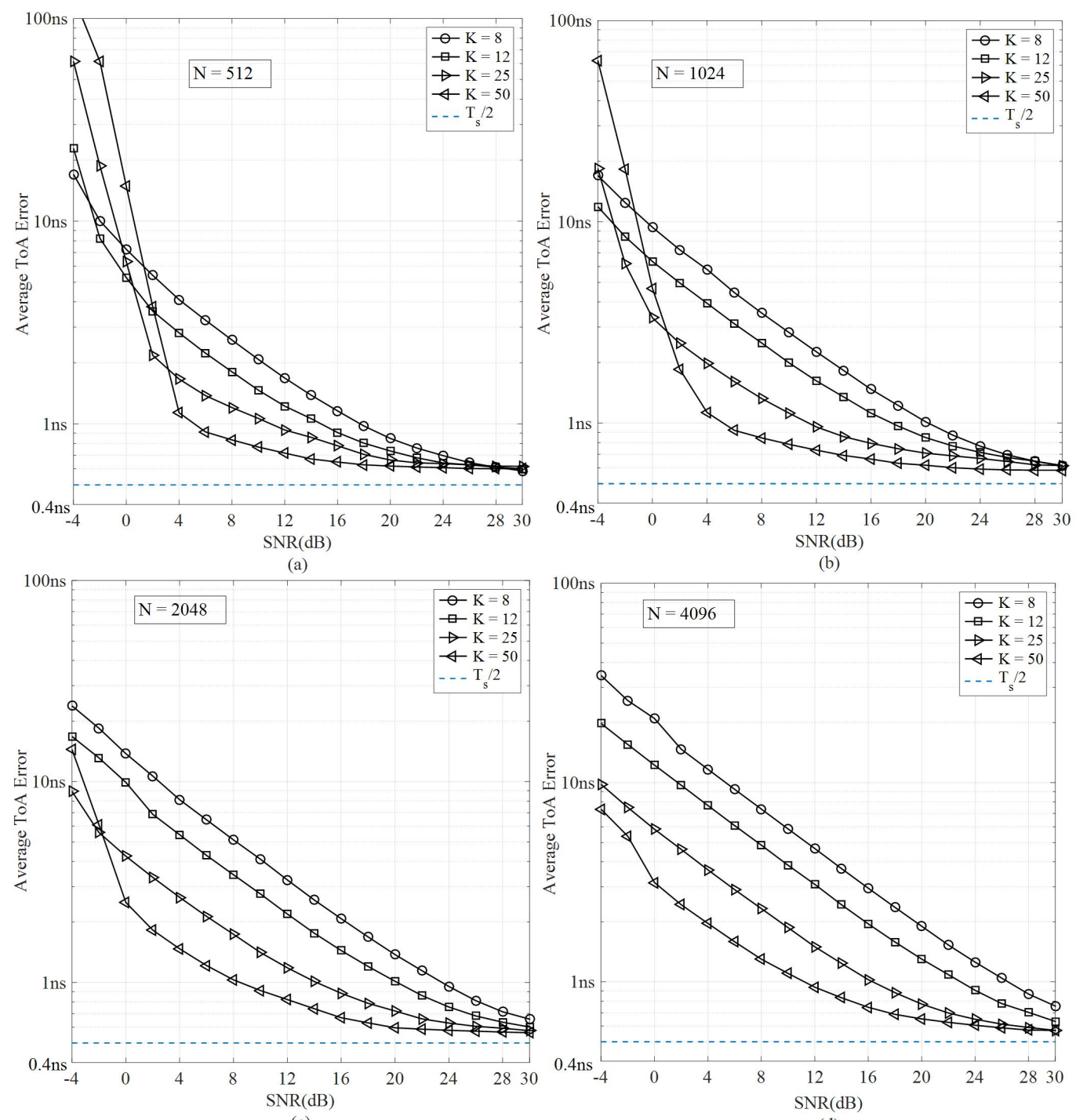

(c)

(d)

Figure 4.6: Impact of the number of allocated subcarriers and transmitted signal length on the estimated ToA average error in airborne to underwater channel, (a) $N=512$, (b) $N=1024$, (c) $N=2048$, and (d) $N=4096$.

improves the ToA estimation performance. Here, a one tap channel is considered as shown in Table 1, because Fig. 4.6 represents the simulations for underwater where we only consider one path between transmitter and receiver as the effect of other paths due to path loss is ignorable. However, different results are observable for human body in 4.5 due to the impact of multipath channels. Here, per Table 1, 
we consider a 5 taps channel, an assumption that is mainly made based on studies conducted in [126], [127] and [128]. Thus, as the bandwidth of transmitted signal increases, i.e., moving from Fig. 4.5(a) to Fig. 4.5(d), the channel frequency selectivity effects becomes more sever that inversely impact ToA estimation. Therefore, based on the number of resolvable paths, the channel bandwidth should be properly selected to maintain the best ToA estimation performance in a multipath channels. This study can form a good problem for our future research.

The same results can be observed for underground medium depicted in Figs. 4.7(a)(d). Here, the same parameters and channel proposed in Section 4.3.1 are applied. As shown in Figs. 4.7(a)-(d), the proposed technique performs accurately at medium to high SNR regimes specifically for large number of allocated subcarriers and waveform length. Comparing Figs. 4.6 and 4.7 it can be observed that the average ToA error converges to the $T_{s} / 2$ which is the minimum average error achievable via coarse ToA estimation. Moreover, moving from Fig. 4.6(a) to Fig. 4.6(d), and Fig. 4.7(a) to Fig. 4.7(d) we observe that increasing the transmitted signal bandwidth improves ToA estimation error. This observation is consistent with our expectation that increasing bandwidth in a flat fading channel improves the ToA estimation performance. Here, a one tap channel is considered as shown in Table 1, because Fig. 4.6 and 4.7 represent the simulations for underwater and underground where we only consider one path between transmitter and receiver as the effect of other paths due to path loss is ignorable. 

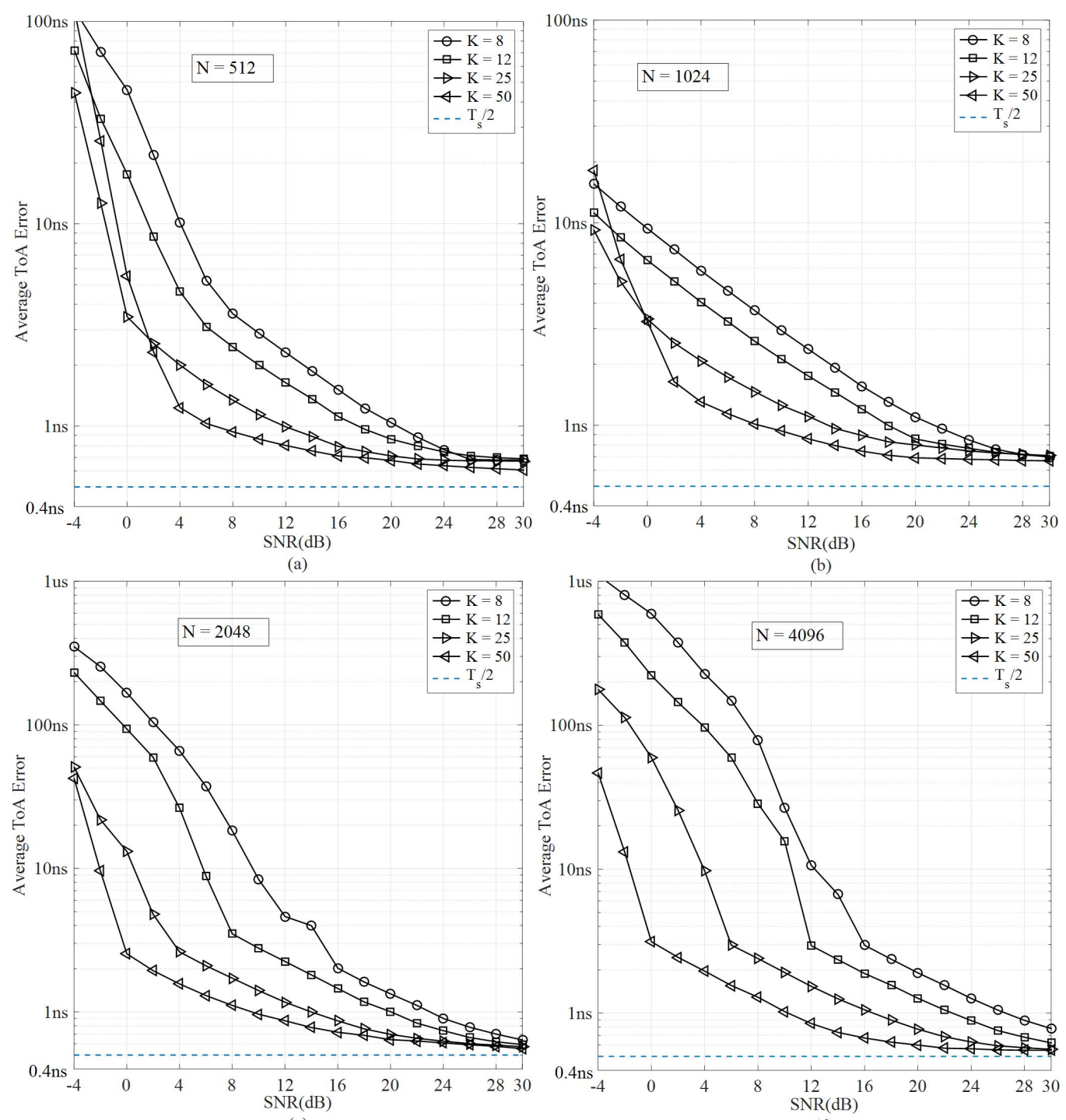

Figure 4.7: Impact of the number of allocated subcarriers and transmitted signal length on the estimated ToA average error in underground channel, (a) $N=512$, (b) $N=1024$, (c) $N=2048$, and (d) $N=4096$.

\subsection{Conclusion}

In this chapter, a novel technique for high resolution ToA estimation in NH media consisting of frequency dispersive sub-media via OFDMA subcarriers is proposed. 
The proposed technique estimates the ToA in two steps. First the frame containing the entire received signal is detected, then the high resolution ToA is proceed by converting the detected frame into frequency domain. The performance of the proposed technique is evaluated via simulations considering human body and airborne to underwater scenarios as two NH media with different physical characteristic according to realistic propagation and channel models. Simulation results indicate that the proposed technique offers high resolution ToA estimation specially for large number of allocated subcarriers with long length for low to high SNR regimes.

Here, the proposed technique approximates the overall imposed delays into subcarriers by a line and estimates the delay corresponding to the spectrum center. Applying the same idea, the slope of approximated line can be estimated to reveal the imposed delays into each subcarrier and equalize them in time domain. This enables very high data rate transmission via OFDM in $\mathrm{NH}$ media. Moreover, the proposed technique opens a new research area in localization and scanning technologies such as LiDAR and GPR in frequency dispersive NH media where no resolution restriction is needed to prevent frequency dispersion of ultra wide band waveforms. 


\section{Chapter 5}

\section{Range Measurements in}

\section{Non-homogenous, Frequency}

\section{Dispersive Channels ${ }^{1}$}

In Chapter 4 the proposed technique for high resolution ToA estimation in $\mathrm{NH}$ media is discussed. However, the proposed ToA measurements cannot be mapped into the actual range (straight-line distance) between the transmitter and receiver. In this chapter range measurement in $\mathrm{NH}$ media is addressed utilizing the proposed ToA measurements in Chapter 4. Here, the proposed ToA measurement technique

\footnotetext{
${ }^{1}$ The material contained in this chapter is submitted for publication in IEEE Transaction on Geoscience and Remote Sensing, July 2016
} 
exploits different carrier frequencies combined with DoA measurements to construct a system of linear equations as a function of the thickness of available sub-media. Once the thicknesses of available sub-media are estimated, the straight-line range between transmitter and receiver is achievable, accordingly.

In order to investigate the feasibility of the proposed range measurement technique, underwater-airborne channels and underground channel consisting several layers with different water contents exploiting wideband microwave signals are considered as examples of NH media. The proposed $\mathrm{NH}$ media are simulated according to the state-of-the-art channel and propagation models for sea water [125] and underground [129, 130, 131]. Simulation results prove the feasibility and efficiency of the proposed technique in terms of ToA resolution and ranging error compared to the allocated bandwidth of the transmitted waveform.

The rest of this chapter is organized as follow. Section 5.1 discusses the system model. Section 5.2 details the proposed range measurement technique. Simulation results and discussions are proposed in Section 5.3, and finally Section 5.4 concludes this chapter. 


\subsection{System Model}

Due to varying electro-magnetic (EM) features in NH media, regardless of the error imposed by EM dispersion, multipath and noise, the ToA measurement proposed in Section 4.2 cannot reveal range between the transmitter and receiver. Figure 5.1 depicts an example where there is an NH channel between the transmitter and the receiver. Here, the addition of propagation path vectors at each sub-media $\left(r_{i}\right)$ is greater than the straight-line propagation path $(r)$ between the transmitter and receiver.

Moreover, the propagation speed at each medium $\left(v_{i}\right)$ is lower than the free space speed of light $(c)$, which leads to higher measured ToA. In addition, based on Snell's law, the angle of the EM wave changes at the media boundary. Thus, there is no

guarantee that the last measured incident angle at the receiver $\hat{\theta}_{M}$ will be equal to the straight-line propagation angle, $\theta$. Hence, a straight-line range measurement procedure needs to be applied exploiting multiple measured ToA.

Figure 5.1 depicts the 3D model for signal propagation in $\mathrm{NH}$ media. Here, we intend to estimate the range between transmitter and receiver which is the straightline distance referred as $r$ in Fig. 5.1. The system model for estimated ToA via 
Algorithm 4, can be written as a function of propagated ranges such that:

$$
\hat{\tau}_{0}=\frac{1}{c} \sum_{m=1}^{M} r_{m} \sqrt{\varepsilon_{r, m}}+v_{\tau},
$$

where $\hat{\tau}_{0}, r_{m}, \varepsilon_{r, m}$ and $v_{\tau}$ are the estimated ToA, the range of propagation path and the relative permittivity in $m^{\text {th }}$ sub-media, and ToA measurement error, respectively, and $c$ and $M$ are defined in 4.15). It can be observed that the proposed system model in (5.1) cannot be solved for desired range $r$, regarding $M$ available unknowns $\left\{r_{m}\right\}_{m=1}^{M}$. In order to address this problem, we propose to exploit a frequencytest (FT) scheme by repeating the ToA measurement scenario applying $Q$ different carrier frequencies. Employing this technique, each ToA measurement provides an independent equation as a function of propagated ranges as the EM features of available sub-media change by the applied carrier frequency. The $q^{\text {th }}$ measured ToA can be written as:

$$
\hat{\tau}_{0}^{(q)}=\frac{1}{c} \sum_{m=1}^{M} r_{m}^{(q)} \sqrt{\varepsilon_{r, m}^{(q)}}+v_{\tau}^{(q)},
$$

where $\hat{\tau}_{0}^{(q)}, r_{m}^{(q)}, \varepsilon_{r, m}^{(q)}$ and $v_{\tau}^{(q)}$ are the estimated ToA, the range of propagation path and relative permittivity in $m^{\text {th }}$ sub-media and ToA measurement error, all at $q^{\text {th }}$ measurement, respectively, and $c$ and $M$ are defined in 4.15. It can be observed that each equation in 5.2 contains a different set of unknowns or $r_{m}^{(q)}$ for $m=1,2, \ldots, M$ and $q=1,2, \ldots, Q$ which leads to a set of $Q$ independent equations containing $M Q$ unknowns. However, these unknowns can be replaced by the 


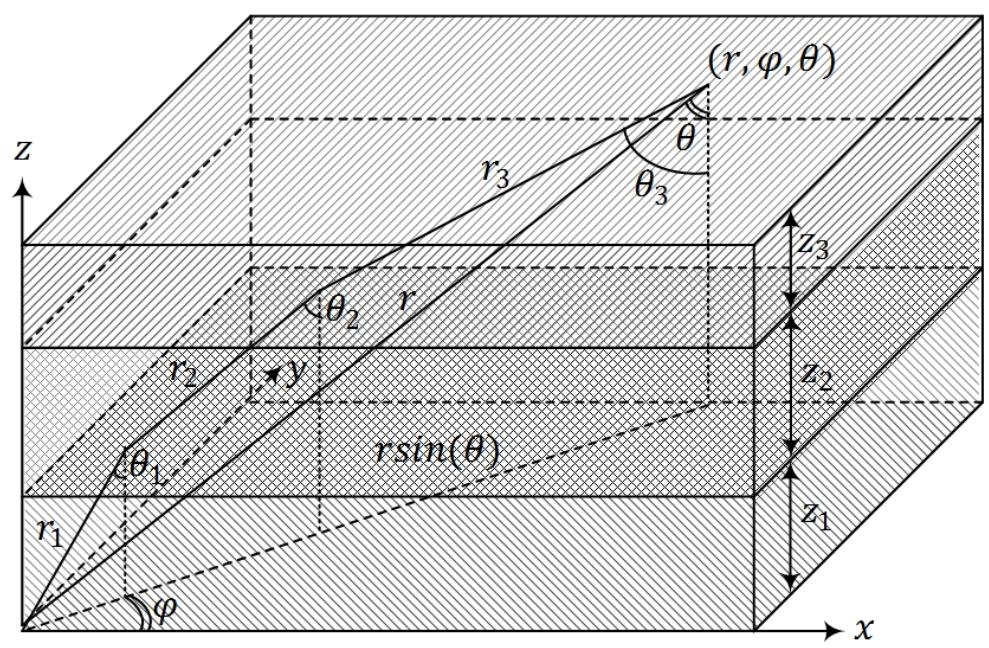

Figure 5.1: 3D model of signal propagation in NH media.

thickness of available sub-media to construct a set of $Q$ independent equations as a function of $M$ available unknowns $z_{m}$ for $m=1,2, \ldots, M$ as follow.

Applying $r_{m}^{(q)}=z_{m} \cos \left(\theta_{m}^{(q)}\right)$, where $\theta_{m}^{(q)}$ is the incident angle of $m^{\text {th }}$ sub-media at $q^{\text {th }}$ carrier frequency, Snell's law $\left(\sin \left(\theta_{m}^{(q)}\right) \sqrt{\varepsilon_{r, m+1}^{(q)}}=\sin \left(\theta_{m+1}^{(q)}\right) \sqrt{\varepsilon_{r, m}^{(q)}}\right)$, and $\cos \left(\sin ^{-1}(x)\right)=\sqrt{1-x^{2}}, 5.2$ can be written as:

$$
\hat{\tau}_{0}^{(q)}=\frac{1}{c} \sum_{m=1}^{M} \frac{z_{m} \sqrt{\varepsilon_{r, m}^{(q)} \varepsilon_{r, M}^{(q)}}}{\sqrt{\varepsilon_{r, M}^{(q)}-\sin ^{2}\left(\theta_{M}^{(q)}\right) \varepsilon_{r, m}^{(q)}}}+v_{\tau}^{(q)}
$$

where, $\hat{\tau}_{0}^{(q)}, \varepsilon_{r, m}^{(q)}$ and $v_{\tau}^{(q)}$ are defined in $(5.2), z_{m}$ denotes the thickness of $m^{t h}$ submedia, and $\theta_{M}^{(q)}$ denotes the DoA corresponding to the $q^{\text {th }}$ measurement at $M^{\text {th }}$ sub-media (last sub-medium including receiver) as shown in Fig. 5.1. Considering (5.3), it can be observed that the set $Q$ measured ToA can be represented as linear 
combination of $M$ available sub-media given the measured DoA at each measurement step. Here, it is assumed that receiver enjoys array antenna which allows DoA estimation recrating state-of-the art techniques such as Root-MUSIC [139] and ESPRIT [71]. These approaches are applicable to NH media consisting of frequency dispersive channels as they utilize a single subcarrier which is free from frequency dispersion. For more information about DoA estimation methods and approaches, we refer readers to $[73]$.

\subsection{Proposed Technique}

Considering Gaussian distribution with zero mean and variance $\sigma_{v}^{2}$ for ToA measurements noise, the ML estimation of thickness of available sub-media can be derived by solving the following optimization problem:

$$
\underset{z_{1}, \ldots, z_{M}}{\operatorname{argmin}} \sum_{q=1}^{Q} \frac{1}{\sigma_{v}^{2}}\left|\hat{\tau}_{0}^{(q)}-\frac{1}{c} \sum_{m=1}^{M} z_{m} \gamma_{m}^{(q)}\right|^{2}
$$

for

$$
\gamma_{m}^{(q)}=\frac{\sqrt{\varepsilon_{r, m}^{(q)} \varepsilon_{r, M}^{(q)}}}{\sqrt{\varepsilon_{r, M}^{(q)}-\sin ^{2}\left(\theta_{M}^{(q)}\right) \varepsilon_{r, m}^{(q)}}}
$$


where $\hat{\tau}_{0}^{(q)}$ and $\varepsilon_{r, m}^{(q)}$, and $\theta_{M}^{(q)}$ and $z_{m}$ are defined in 5.2 and $(5.3)$, respectively. The least square solution for the proposed ML estimator in (5.4), can be represented by:

$$
\hat{\mathbf{z}}=\left(\mathbf{\Upsilon}^{T} \Upsilon\right)^{-1} \mathbf{\Upsilon}^{T} \tau
$$

where the $(.)^{-1}$ and $(.)^{T}$ operands denote operands for matrix inversion and transpose, respectively, and $\hat{\mathbf{z}}=\left[\hat{z}_{1}, \hat{z}_{2}, \ldots, \hat{z}_{M}\right]^{T}, \tau=\left[\hat{\tau}_{0}^{(1)}, \hat{\tau}_{0}^{(2)}, \ldots, \hat{\tau}_{0}^{(Q)}\right]^{T}$ and $\Upsilon=$ $\left[\Upsilon_{1}, \Upsilon_{2}, \ldots, \Upsilon_{M}\right]^{T}$, for:

$$
\Upsilon_{m}=\left[\gamma_{m}^{(1)}, \gamma_{m}^{(2)}, \ldots, \gamma_{m}^{(Q)}\right]^{T}
$$

where $\hat{z}_{m}$ denote the estimation of $m^{t h}$ sub-media's thickness, and $\hat{\tau}_{0}^{(q)}$ and $\gamma_{m}^{(q)}$ are defined in (5.2) and (5.5), respectively. Given the estimated thickness of available sub-media using (5.6), it can be shown that (see Appendix B), the propagation range between transmitter and receiver is achievable via $\hat{r}=\frac{1}{Q} \sum_{q=1}^{Q} \hat{r}^{(q)}$ where:

$$
\hat{r}^{(q)}=D \sqrt{1+\left[\frac{1}{D} \sum_{m=1}^{M} \frac{\hat{z}_{m} \sin \left(\theta_{M}^{(q)}\right) \sqrt{\varepsilon_{r, m}^{(q)}}}{\sqrt{\varepsilon_{r, M}^{(q)}-\sin ^{2}\left(\theta_{M}^{(q)}\right) \varepsilon_{r, m}^{(q)}}}\right]^{2}}
$$

for $D=\sum_{m=1}^{M} z_{m}$. Moreover, $\varepsilon_{r, m}^{(q)}$ and $\theta_{M}^{(q)}$, and $\hat{z}_{m}$ are defined in 5.2 and 5.6, respectively. In the next section the performance of high resolution ToA estimation and range measurement are discussed considering different $\mathrm{NH}$ channels. 


\subsection{Simulations Result and Discussions}

This section discusses the simulation results of proposed range estimation in airborne-underwater and underground media. Details of applied NH media and transmitted waveform are introduced in sub-section 4.1. Figures 5.2(a) and (b) depict the impact of the number of applied measurements (ToA and DoA) and average ToA measurements error given perfect DoA measurements on the estimated range for underwater-airborne and underground channels, respectively considering $N=4096$ and $K=50$. It is observed (see Figs. 4.6(d) and 4.7(d)) that the average ToA estimation error is less than or equal to 5 ns for $S N R(d B) \geq 0$ for $N=4096$ and $K=50$. Therefore, applying the same parameters $(N=4096$ and $K=50)$, here we have evaluated the average range error vs the average ToA measurements error less than or equal 5ns. It is observed that the proposed technique offers the average range error less that $1 \mathrm{~m}$ for underwater-airborne and underground channels at ToA measurements error around or less than 2 ns which is achievable at $S N R(d B) \geq 4$. Moreover, it is observed that the average ranging error decreases by increasing the number of applied measurements as expected.

Figures 5.3(a) and (b) depict the impact of the number of applied measurements (ToA and DoA) and average DoA measurements error given perfect ToA measurements on the estimated range for underwater-airborne and underground channels, 
respectively considering $N=4096$ and $K=50$. It is observed that the proposed technique offers proper range estimation even at imperfect DoA measurements. Furthermore, it is observed that the average ranging error is decreased by increasing the number of applied measurements for all applied DoA measurements error as expected.

Comparing Figs. 5.2 and 5.3 the feasibility of the proposed technique on different values of desired range can be evaluated. As shown in Figs 5.2 (a) and (b), the ranging error for two different range values $(30 \mathrm{~m}$ and $150 \mathrm{~m}$ for underwater-airborne and $10 \mathrm{~m}$ and $25 \mathrm{~m}$ for underground) are the same at perfect DoA measurements. However, it is observed that the ranging error corresponding to larger range values are higher as shown in Figs 5.3(a) and (b). This is due to the impact of DoA on ranging utilizing the estimated thicknesses according to the final range estimation equation proposed in (5.8). Therefore, it should be noted that higher precision DoA measurements are required in scenarios with larger range between transmitter and receiver. 


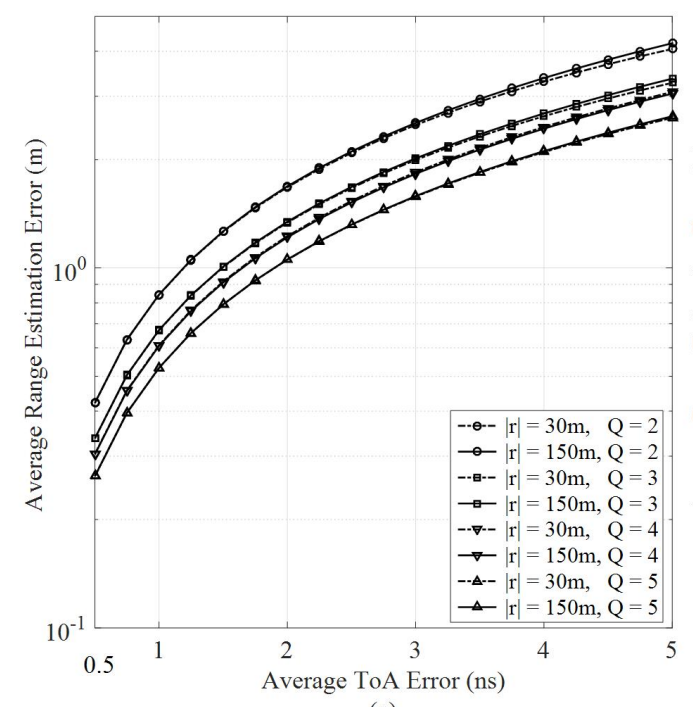

(a)

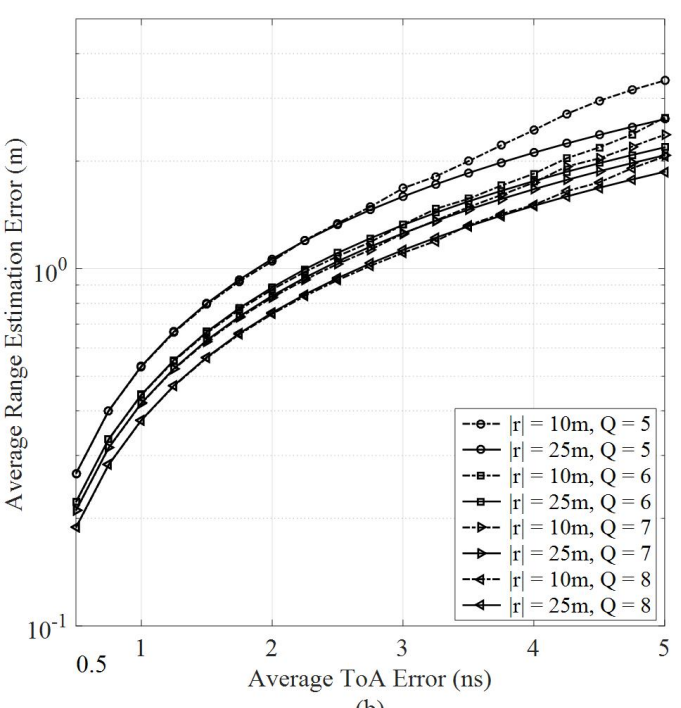

(b)

Figure 5.2: Impact of the number of applied measurements $Q$ and ToA measurements error on the estimated range, (a) underwater-airborne channel, (b) underground channel.

\subsection{Conclusion}

In this chapter a novel ToA/DoA aimed range layer thickness and range measurements are proposed. Considering multiple ToA/DoA measurements recruiting different carrier frequencies, here, a set of linear equations is constructed which is solved for available sub-media's thickness and hence, straight-line range between transmitter and receiver. The performance of the proposed technique is evaluated via simulations considering underwater-airborne and multi-layers underground scenarios as two NH media with different physical characteristic according to realistic propagation and channel models. Simulation results indicate that the proposed technique offers precise ranging specially for large number of allocated subcarriers 


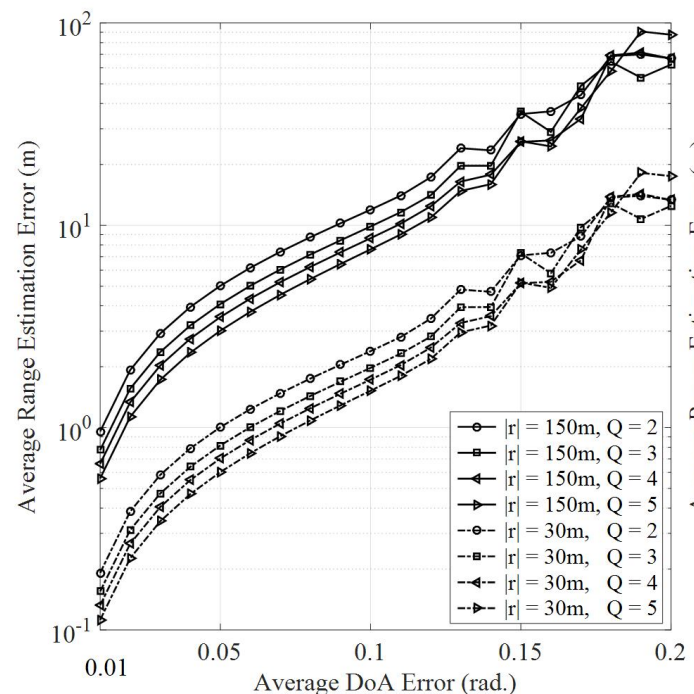

(a)

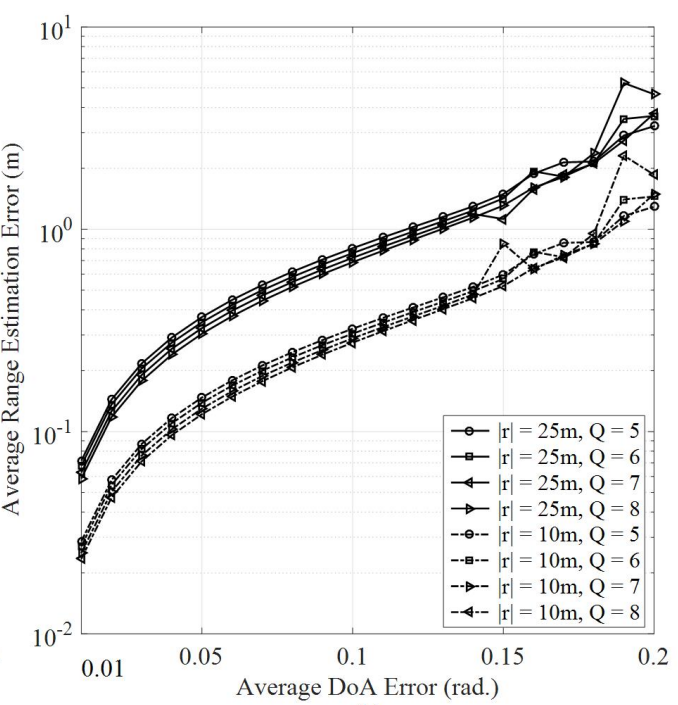

(b)

Figure 5.3: Impact of the number of applied measurements $Q$ and DoA measurements error on the estimated range, (a) underwater-airborne channel, (b) underground channel.

with long length for low to high SNR regimes.

The proposed technique opens a new research area in localization and scanning technologies such as LiDAR and GPR in frequency dispersive NH media where no resolution restriction is needed to prevent frequency dispersion of ultra wide band waveforms. Moreover, the proposed ToA estimation technique approximates the overall imposed delays into subcarriers by a line and estimates the delay corresponding to the spectrum center. Applying the same idea, the slope of the approximated line is estimated to reveal the imposed delays into each subcarrier and equalize them in time domain. This enables very high data rate transmission exploiting OFDMA subcarriers in frequency dispersive $\mathrm{NH}$ media such as underwater or underground channels. 



\section{Chapter 6}

\section{Conclusions and Future Works}

In this chapter a summery of conclusions and future works of the proposed range measurement technique in $\mathrm{NH}$ media is proposed.

\subsection{Conclusions}

In this thesis, the problem of ToA based straight-line range measurement in $\mathrm{NH}$ media containing frequency dispersive sub-media is proposed. Here, we show that traditional ToA estimation techniques are not feasible due to frequency dispersion of 
transmitted waveform. Therefore, we introduce using bandlimited waveform meanwhile the low resolution of ToA estimation imposed by limited bandwidth is compensated recruiting the MRLD receiver. Although the proposed technique provides very good result in terms of increasing ToA resolution, however, the proposed resolution is not enough for some NH application such as human body.

In order to address this issue a novel technique for ToA estimation in NH media is proposed incorporating OFDMA subcarriers. In the proposed technique, a waveform constructed of symmetric OFDMA subcarrier is transmitted through the $\mathrm{NH}$ channel. Here, we show that the delay imposed by frequency dispersion of media can be approximated by a line which allows reduction of unknowns (i.e. delays imposed to transmitted sub-carriers). Exploiting these approximation, we proposed a precise method which estimates the delay of the subcarrier corresponding to the center of waveform spectrum. Simulation result in three different NH media indicates that the applied approximation are thoroughly feasible which leads to perfect ToA estimation from medium to high SNR regimes.

Although the introduced ToA estimation proposes enough resolution, they cannot be mapped into the actual range between the transmitter and receiver as the propagation path of transmitted waveform is not an straight-line. In order to resolve this issue a novel technique which enjoys ToA/DoA estimation is proposed. Here, the proposed ToA measurement technique exploits different carrier frequencies combined 
with DoA measurements to construct a system of linear equations as a function of the thickness of available sub-media. Once the thicknesses of available sub-media are estimated, the straight-line range between transmitter and receiver is estimated, accordingly. Simulation results confirm that the proposed technique propose good ranging resolution given noisy ToA and DoA measurements for different NH media.

\subsection{Future Works}

Although the proposed range measurement technique covers all requirements to accomplish, here we propose a summery of open problems which can be defined as extension of the proposed technique for further research.

\section{$\dagger$ Subcarrier delay estimation for OFDM based data transmission in} NH media: the proposed ToA estimation technique approximates the overall imposed delays into subcarriers by a line and estimates the delay corresponding to the spectrum center. Applying the same idea, the slope of the approximated line is estimated to reveal the imposed delays into each subcarrier and equalize them in time domain. This enables very high data rate transmission exploiting OFDMA subcarriers in frequency dispersive NH media such as underwater or underground channels. 


\section{$\dagger$ Optimum carrier frequency selection for layer thickness estimation:}

As discussed in Section 5.2 , the procedure of straight-line range measurements requires multiple (depends on the number of available sub-media) ToA/DoA measurements utilizing different carrier frequency. These measurements construct a system of linear equations as a function of the thickness of available sub-media. However, if the carrier frequencies are selected non-properly (for instance too close to each other) the measurements are dependent which leads to ill-conditioned measurement matrix proposed in (5.7). Here, a tradeoff between the possible carrier frequency range and the error imposed by the condition number of measurements matrix can be defined and solved.

$\dagger$ Investigating the impact of carrier frequency and sampling time offset and their estimation: Recruiting OFDMA sub-carriers requires addressing the carrier frequency and sampling time offset estimation due to high sensitivity of OFDM to frequency and time offset. Here, the proposed results are considered full sampling time and carrier frequency synchronization which is not a feasible assumption in practice. Therefore, further investigation on the impact of time and frequency offset on the proposed ToA estimation and hence the range measurement techniques is required.

$\dagger$ Cooperative Range Measurements: In the proposed ToA estimation and ranging techniques, a pair of transmitter and receiver is considered. However, 
in majority of WSN applications multiple sensor nodes are available. Considering the proposed range measurements technique which applies several different carrier frequencies, here an open problem which proposes a carrier frequency hopping scheme for cooperative range measurement is vital. 



\section{References}

[1] Akyildiz, I. F.; Melodia, T.; Chowdury, K. R. Wireless Communications, IEEE 2007, 14(6), 32-39.

[2] Akyildiz, I. F.; Su, W.; Sankarasubramaniam, Y.; Cayirci, E. Computer Networks 2002, 38(4), 393-422.

[3] Sohraby, K.; Minoli, D.; Znati, T. Wireless sensor networks: technology, protocols, and applications; John Wiley and Sons, 2007.

[4] Dardari, D.; Conti, A.; Buratti, C.; Verdone, R. Mobile Computing, IEEE Transactions on $\mathbf{2 0 0 7 , 6 ( 7 ) , 7 9 0 - 8 0 2 . ~}$

[5] Hui, Y.; Yong, Q.; Gefei, F.; Hui, C. Sensors Journal, IEEE 2013, 13(2), $556-562$.

[6] Jelicic, V.; Magno, M.; Brunelli, D.; Paci, G.; Benini, L. Sensors Journal, IEEE 2013, 13(1), 328-338. 
[7] Jianfa, X.; Zhenzhou, T.; Xiaoqiu, S.; Lei, F.; Huaizhong, L. In Mobile Adhoc and Sensor Networks (MSN), 2011 Seventh International Conference on, pages $28-35$.

[8] Wood, A.; Stankovic, J. A.; Virone, G.; Selavo, L.; Zhimin, H.; Qiuhua, C.; Thao, D.; Yafeng, W.; Lei, F.; Stoleru, R. Network, IEEE 2008, 22(4), 26-33.

[9] Xianghui, C.; Jiming, C.; Yang, X.; Youxian, S. Industrial Electronics, IEEE Transactions on 2010, 57(11), 3596-3605.

[10] Ghataoura, D. S.; Mitchell, J. E.; Matich, G. E. Communications Magazine, IEEE 2011, 49(10), 90-97.

[11] Kewei, S.; Weisong, S.; Watkins, O. In Electro/information Technology, 2006 IEEE International Conference on, pages 239-244.

[12] Will, H.; Schleiser, K.; Schiller, J. In Local Computer Networks, 2009. LCN 2009. IEEE 34th Conference on, pages 834-841.

[13] Huang, Y. M.; Hsieh, M. Y.; Chao, H. C.; Hung, S. H.; Park, J. H. Selected Areas in Communications, IEEE Journal on 2009, 27(4), 400-411.

[14] Lo.; x.; pez, G.; Custodio, V.; Moreno, J. I. Information Technology in Biomedicine, IEEE Transactions on 2010, 14(6), 1446-1458.

[15] Wolf, L.; Saadaoui, S. In Consumer Communications and Networking Conference, 200\%. CCNC 2007. 4th IEEE, pages 722-726. 
[16] Gyselinckx, B.; Van Hoof, C.; Ryckaert, J.; Yazicioglu, R. F.; Fiorini, P.; Leonov, V. In Custom Integrated Circuits Conference, 2005. Proceedings of the IEEE 2005, pages 13-19.

[17] Ivanov, S.; Botvich, D.; Balasubramaniam, S. Consumer Electronics, IEEE Transactions on 2012, 58(2), 284-292.

[18] Mitra, U.; Emken, B. A.; Sangwon, L.; Ming, L.; Rozgic, V.; Thatte, G.; Vathsangam, H.; Zois, D.; Annavaram, M.; Narayanan, S.; Levorato, M.; SpruijtMetz, D.; Sukhatme, G. Communications Magazine, IEEE 2012, 50(5), 116125.

[19] Guevara, J.; Barrero, F.; Vargas, E.; Becerra, J.; Toral, S. Intelligent Transport Systems, IET 2012, 6(2), 177-186.

[20] Pascale, A.; Nicoli, M.; Deflorio, F.; Dalla Chiara, B.; Spagnolini, U. Intelligent Transport Systems, IET 2012, 6(1), 67-77.

[21] Zhou, J.; Chen, C. L. P.; Chen, L.; Zhao, W. Intelligent Transportation Systems, IEEE Transactions on 2013, PP(99), 1-10.

[22] Khan, A.; Jenkins, L. In Communication Systems Software and Middleware and Workshops, 2008. COMSWARE 2008. 3rd International Conference on, pages $2-8$. 
[23] Khedo, K.; Perseedoss, R.; Mungur, A. International Journal of Wireless and Mobile Networks 2010, 2(2), 31-45.

[24] afchi, A. R.; Shu Ting, G.; Reza Zekavat, S. A. Sensors Journal, IEEE 2014, $14(11), 3791-3799$.

[25] Shu Ting, G.; Reza Zekavat, S. A.; Pahlavan, K. Sensors Journal, IEEE 2014, $14(11), 3819-3829$.

[26] Prabhulkar, S.; Chen-Zhong, L. In Bioinformatics and Biomedical Engineering , 2009. ICBBE 2009. 3rd International Conference on, pages 1-4.

[27] Tsalach, A.; Steinberg, I.; Gannot, I. Time Difference of Arrival Based Cancer Tumor Localization Using Magnetic Nano-particles Induced Acoustic Signals Thesis, 2012.

[28] Zheng, G.; Patolsky, F.; Cui, Y.; Wang, W. U.; Lieber, C. M. Nature biotechnology 2005, 23(10), 1294-1301.

[29] De Jong, W. H.; Borm, P. J. International journal of nanomedicine 2008, 3(2), 133.

[30] Gupta, R. B.; Kompella, U. B. Nanoparticle technology for drug delivery; Taylor and Francis New York, 2006.

[31] Moses, M. A.; Brem, H.; Langer, R. Cancer cell 2003, 4(5), 337-341. 
[32] Nakano, T.; Moore, M. J.; Okaie, Y.; Enomoto, A.; Suda, T. In Communications Workshops (ICC), 2013 IEEE International Conference on, pages 809-812.

[33] Singh, R.; Lillard Jr, J. W. Experimental and Molecular Pathology 2009, 86(3), 215-223.

[34] Yih, T.; AlFandi, M. Journal of cellular biochemistry 2006, 97(6), 1184-1190.

[35] Cayirci, E.; Tezcan, H.; Dogan, Y.; Coskun, V. Ad Hoc Networks 2006, 4(4), 431-446.

[36] Xiaoguang, N.; Xi, H.; Ze, Z.; Yuhe, Z.; Changcheng, H.; Li, C. In Global Telecommunications Conference, 200\%. GLOBECOM '0\%. IEEE, pages 12911295.

[37] Yang, W.; Huang, Y. In Networking, 200\%. ICN'0\%. Sixth International Conference on, pages 13-13. IEEE.

[38] Enyang, X.; Zhi, D.; Dasgupta, S. Signal Processing, IEEE Transactions on 2011, 59(6), 2887-2897.

[39] Bing, W.; Wu, C.; Xiaoli, D. In Ubiquitous Positioning Indoor Navigation and Location Based Service (UPINLBS), 2010, pages 1-8.

[40] Yu-Yi, C.; Yi-Yuan, L. Consumer Electronics, IEEE Transactions on 2009, 55(3), 1295-1299. 
[41] Gang, W.; Kehu, Y. Wireless Communications, IEEE Transactions on 2011, 10(5), 1389-1395.

[42] Tomic, S.; MARKO, B.; Dinis, R.; LIPOVAC, V.; GORAN, D. C. In 11th WSEAS International Conference on Applied Electromagnetics, Wireless and Optical Communications.

[43] Xinrong, L. Vehicular Technology, IEEE Transactions on 2007, 56(6), 38073817.

[44] Yeong-Sheng, C.; Tai-Lin, C.; Yi-Chen, H. In Wireless Personal Multimedia Communications (WPMC), 2012 15th International Symposium on, pages $341-347$.

[45] Hong, S.; Zhi, D.; Dasgupta, S.; Chunming, Z. Signal Processing, IEEE Transactions on 2014, 62(8), 1938-1949.

[46] Kehu, Y.; Gang, W.; Zhi-Quan, L. Signal Processing, IEEE Transactions on 2009, 57(7), 2775-2784.

[47] Qingjiang, S.; Chen, H.; Hongyang, C.; Lingge, J. Signal Processing, IEEE Transactions on $\mathbf{2 0 1 0}, 58(6), 3328-3340$.

[48] Srirangarajan, S.; Tewfik, A. H.; Luo, Z.-Q. Wireless Communications, IEEE Transactions on $\mathbf{2 0 0 8 , 7 ( 1 2 ) , 4 8 8 6 - 4 8 9 5 . ~}$

[49] Yu, K.; Guo, Y. J.; Hedley, M. Signal Processing, IET 2009, 3(2), 106-118. 
[50] Enyang, X.; Zhi, D.; Dasgupta, S. Mobile Computing, IEEE Transactions on 2011, 10(9), 1276-1282.

[51] Lui, K. W. K.; Chan, F. K. W.; So, H. C. Signal Processing, IEEE Transactions on $\mathbf{2 0 0 9}, 57(4), 1630-1633$.

[52] Shi, Z.; Lirong, R.; Jun, X.; Jindong, T. In Control, Automation, Robotics and Vision, 2006. ICARCV'06. 9th International Conference on, pages 1-6.

[53] Wendeberg, J.; Hoflinger, F.; Schindelhauer, C.; Reindl, L. In Indoor Positioning and Indoor Navigation (IPIN), 2011 International Conference on, pages $1-10$.

[54] Cooperative localization using angle of arrival measurements in non-line-ofsight environments. Ananthasubramaniam, B.; Madhow, U. 2008.

[55] Baoli, Z.; Fengqi, Y. Consumer Electronics, IEEE Transactions on 2010, $56(4), 2208-2216$.

[56] Biswas, P.; Aghajan, H.; Yinyu, Y. In Signals, Systems and Computers, 2005. Conference Record of the Thirty-Ninth Asilomar Conference on, pages 220224.

[57] Biswas, P.; Aghajan, H.; Ye, Y. In 39th Asilomar Conference on Signals, Systems and Computers. Citeseer. 
[58] Rong, P.; Sichitiu, M. L. In Sensor and Ad Hoc Communications and Networks, 2006. SECON '06. 2006 3rd Annual IEEE Communications Society on, Vol. 1, pages 374-382.

[59] Alippi, C.; Vanini, G. In Pervasive Computing and Communications Workshops, 2006. PerCom Workshops 2006. Fourth Annual IEEE International Conference on, pages 5 pp.-305.

[60] Harley, J. B.; Moura, J. M. F. In Acoustics, Speech and Signal Processing (ICASSP), 2013 IEEE International Conference on, pages 4071-4075.

[61] Mirbach, M.; Menzel, W. In Ultra-Wideband (ICUWB), 2012 IEEE International Conference on, pages 328-332.

[62] Reddy, V. V.; Boon Poh, N.; Khong, A. W. H. In Information, Communications and Signal Processing (ICICS) 2013 9th International Conference on, pages $1-5$.

[63] Sahota, H.; Kumar, R. In Networking, Sensing and Control (ICNSC), 2014 IEEE 11th International Conference on, pages 203-208.

[64] Thanasopoulos, I.; Avaritsiotis, J. Science, Measurement and Technology, IET 2011, 5(4), 125-133.

[65] Pahlavan, K.; Ye, Y.; Fu, R.; Khan, U. International Journal of Embedded and Real-Time Communication Systems (IJERTCS) 2012, 3(3), 18-37. 
[66] Chandrasekhar, V.; Seah, W. K.; Choo, Y. S.; Ee, H. V. In Proceedings of the 1st ACM international workshop on Underwater networks, pages 33-40. ACM.

[67] Che, X.; Wells, I.; Dickers, G.; Kear, P.; Gong, X. IEEE Communications Magazine 2010, 48(12), 143-151.

[68] Lele, Q.; Qiang, S.; Tianhong, Y.; Lili, Z.; Yanpeng, S. IEEE Geoscience and Remote Sensing Letters 2014, 11(8), 1315-1319.

[69] Bastard, C. L.; Yide, W.; Baltazart, V.; Derobert, X. IEEE Geoscience and Remote Sensing Letters 2014, 11(4), 873-877.

[70] Daniels, D. J. Ground penetrating radar, Vol. 1; Iet, 2004.

[71] Roy, R.; Paulraj, A.; Kailath, T. In 30th Annual Technical Symposium, pages 94-101. International Society for Optics and Photonics.

[72] Huilin, X.; Chia-Chin, C.; Guvenc, I.; Watanabe, F.; Liuqing, Y. In Communications, 2008. ICC '08. IEEE International Conference on, pages 4191-4196.

[73] Zekavat, R.; Buehrer, R. M. 2011.

[74] Lee, J.-Y.; Scholtz, R. A. Selected Areas in Communications, IEEE Journal on 2002, 20(9), 1677-1683.

[75] Wang, J.; Shen, Z. 2002. 
[76] Li, X.; Pahlavan, K. Wireless Communications, IEEE Transactions on 2004, 3(1), 224-234.

[77] Wang, F.; Zhang, X.; Wang, F. Wireless Personal Communications 2014, pages $1-20$.

[78] Daegun, O.; Sangdeok, K.; Sang-Hun, Y.; Jong-wha, C. Wireless Communications, IEEE Transactions on 2013, 12(7), 3130-3139.

[79] Chang, R. W. Bell System Technical Journal 1966, 45(10), 1775-1796.

[80] Orthogonal frequency multiplex data transmission system. Chang, R. W. 1970.

[81] Saltzberg, B. IEEE Transactions on Communication Technology 1967, 15(6), 805-811.

[82] Weinstein, S.; Ebert, P. IEEE Transactions on Communication Technology $1971,19(5), 628-634$.

[83] Hirosaki, B. IEEE Transactions on Communications 1981, 29(7), 982-989.

[84] IEEE Std 802.11-2007 (Revision of IEEE Std 802.11-1999) 2007, pages 11076.

[85] IEEE Std 802.16m-2011(Amendment to IEEE Std 802.16-2009) 2011, pages $1-1112$. 
[86] Remy, J.; Letamendia, C. LTE Standards pages 1-112.

[87] Li, Y. G.; Stuber, G. L. Orthogonal frequency division multiplexing for wireless communications; Springer Science \& Business Media, 2006.

[88] Hui, T.; Zekavat, S. A. Vehicular Technology, IEEE Transactions on 2007, 56(3), 1307-1320.

[89] Green, M. P. 2005, (contribution 802.15-05-0499-00-004a IEEE 802.15.4a Ranging Subcommittee).

[90] Jamalabdollahi, M.; Zekavat, S. In Aerospace Conference, 2014 IEEE, pages $1-7$.

[91] Bachir, A.; Dohler, M.; Watteyne, T.; Leung, K. K. Communications Surveys and Tutorials, IEEE 2010, 12(2), 222-248.

[92] Pourkhaatoun, M.; Zekavat, S. A. Communications, IET 2011, 5(10), 14401452.

[93] Borbash, S. A.; Ephremides, A.; McGlynn, M. J. Ad Hoc Networks 2007, 5(7), 998-1016.

[94] Kohvakka, M.; Suhonen, J.; Kuorilehto, M.; Kaseva, V.; Hnnikinen, M.; Hmlinen, T. D. Ad Hoc Networks 2009, 7(1), 24-41.

[95] Jun, L.; Dongning, G. In Communication, Control, and Computing, 2008 46th Annual Allerton Conference on, pages 791-797. 
[96] Angelosante, D.; Biglieri, E.; Lops, M. Physical Communication 2010, 3(1), 28-36.

[97] Lin, D. D.; Teng Joon, L. Communications, IEEE Transactions on 2004, 52(4), 612-621.

[98] Zhang, L.; Luo, J.; Guo, D. Performance Evaluation 2013, 70(78), 457-471.

[99] Jun, L.; Dongning, G. In Communication, Control, and Computing, 2009. Allerton 2009. 47th Annual Allerton Conference on, pages 308-313.

[100] Yaacoub, E.; Kadri, A.; Abu-Dayya, A. In Wireless Communications and Mobile Computing Conference (IWCMC), 2011 rth International, pages 18941899.

[101] Qin, F. Technologies to improve the performance of wireless sensor networks in high-traffic applications Thesis, 2012.

[102] Wenjie, X.; Zekavat, S. A. Systems Journal, IEEE 2014, 8(1), 125-130.

[103] Jamalabdollahi, M.; Mirzaeinia, A.; Salari, S. In Advanced Communication Technology (ICACT), 2012 14th International Conference on, pages 832-836.

[104] Cc2500: Low cost, low-power 2.4 ghz rf transceiver. Instruments, T. 2007.

[105] Instruments, T. HYPERLINK" http://focus. ti. com/" http://focus. ti. com 2009. 
[106] Alazzawi, L.; Elkateeb, A. Journal of Computer Systems, Networks, and Communications 2009, 2008.

[107] Jamalabdollahi, M.; Salari, S. Wireless personal communications 2013, 71(2), 1159-1174.

[108] He, H.; Li, J.; Stoica, P. Waveform design for active sensing systems: a computational approach; Cambridge University Press, 2012.

[109] Stoica, P.; Hao, H.; Jian, L. Signal Processing, IEEE Transactions on 2009, $57(4), 1415-1425$.

[110] Jedwab, J. In Sequences and Their Applications-SETA 2004; Springer, 2005; pages $30-55$.

[111] Rapajic, P. B.; Kennedy, R. A. Communications Letters, IEEE 1998, 2(10), 269-270.

[112] Hao, H.; Stoica, P.; Jian, L. Signal Processing, IEEE Transactions on 2009, $57(11), 4391-4405$.

[113] Voltz, P. J.; Hernandez, D. In Position Location and Navigation Symposium, 2004. PLANS 2004, pages 585-591.

[114] Lindenfeld, M. J. Aerospace and Electronic Systems, IEEE Transactions on 2004, 40(3), 851-861. 
[115] Rowe, W.; Stoica, P.; Jian, L. Signal Processing Magazine, IEEE 2014, 31(3), $157-162$.

[116] Benedetto, F.; Giunta, G.; Guzzon, E. In Positioning Navigation and Communication (WPNC), 2011 8th Workshop on, pages 137-142.

[117] Fuchs, J. J. Signal Processing, IEEE Transactions on 1999, 47(1), 237-243.

[118] Ziming, H.; Ma, Y.; Tafazolli, R.; Liu, H. 2010.

[119] Hao, C.; Orlando, D.; Foglia, G.; Ma, X.; Hou, C. Signal Processing Letters, IEEE 2015, 22(9), 1359-1363.

[120] Hamila, R.; Vesma, J.; Renfors, M. Circuits and Systems II: Analog and Digital Signal Processing, IEEE Transactions on 2002, 49(8), 567-576.

[121] Conn, A. R.; Gould, N. I.; Toint, P. L. Trust region methods, Vol. 1; Siam, 2000.

[122] Dardari, D.; Chong, C. C.; Win, M. Z. In Ultra-Wideband, The 2006 IEEE 2006 International Conference on, pages 531-537.

[123] Feng-Xiang, G.; Dongxu, S.; Yingning, P.; Li, V. O. K. Circuits and Systems I: Regular Papers, IEEE Transactions on 2007, 54(9), 1977-1986.

[124] Quazi, A. Acoustics, Speech and Signal Processing, IEEE Transactions on 1981, 29(3), 527-533. 
[125] Pieraccini, M.; Bicci, A.; Mecatti, D.; Macaluso, G.; Atzeni, C. IEEE Transactions on Antennas and Propagation 2009, 57(11), 3612-3618.

[126] Khaleghi, A.; Chavez-Santiago, R.; Balasingham, I. IET Microwaves, Antennas 65 Propagation 2011, 5(15), 1805-1812.

[127] Khaleghi, A.; Cha.; x.; vez Santiago, R.; Liang, X.; Balasingham, I.; Leung, V. C. M.; Ramstad, T. A. In Wireless Pervasive Computing (ISWPC), 2010 5th IEEE International Symposium on, pages 140-145.

[128] Chavez-Santiago, R.; Sayrafian-Pour, K.; Khaleghi, A.; Takizawa, K.; Wang, J.; Balasingham, I.; Li, H.-B. Communications Magazine, IEEE 2013, 51(8), 80-87.

[129] Wagner, N.; Emmerich, K.; Bonitz, F.; Kupfer, K. IEEE Transactions on Geoscience and Remote Sensing 2011, 49(7), 2518-2530.

[130] Vuran, M. C.; Akyildiz, I. F. Physical Communication 2010, 3(4), 245-254.

[131] Akyildiz, I. F.; Sun, Z.; Vuran, M. C. Physical Communication 2009, 2(3), $167-183$.

[132] Jamalabdollahi, M.; Zekavat, S. A. R. Sensors Journal, IEEE 2015, 15(10), $5821-5833$.

[133] Gabriel, C. Compilation of the dielectric properties of body tissues at rf and microwave frequencies Report, DTIC Document, 1996. 
[134] Meissner, T.; Wentz, F. J. IEEE Transactions on Geoscience and Remote Sensing 2004, 42(9), 1836-1849.

[135] Debye, P. J. W. Polar molecules; Chemical Catalog Company, Incorporated, 1929.

[136] Stojanovic, M.; Preisig, J. IEEE Communications Magazine 2009, 47(1), 8489.

[137] Jaruwatanadilok, S. IEEE Journal on Selected Areas in Communications 2008, 26(9), 1620-1627.

[138] Hattab, G.; El-Tarhuni, M.; Al-Ali, M.; Joudeh, T.; Qaddoumi, N. International Journal of Distributed Sensor Networks 2013, 2013.

[139] Barabell, A. J. In Acoustics, Speech, and Signal Processing, IEEE International Conference on ICASSP'83., Vol. 8, pages 336-339. IEEE.

[140] Koorapaty, H. In Vehicular Technology Conference, 2004. VTC 2004-Spring. 2004 IEEE 59th, Vol. 5, pages 2729-2733 Vol.5. 


\title{
Appendix A
}

\section{Proofs and Derivations}

\section{A.1 Probabilities of Miss-Detection and False-}

\author{
Alarm
}

Applying $\tau^{(m)}=k^{*} T_{s}$ into 2.10 for the $n^{\text {th }}$ entry of $\mathbf{y}$ we have:

$$
\begin{aligned}
y_{n}=\mathbf{w}_{n}^{T} \mathbf{r}=\sum_{k=0}^{k^{*}-1} v\left(k T_{s}\right) e^{-j 2 \pi n k / N} & +\sum_{k=k^{*}}^{k^{*}+N-1} e^{-j 2 \pi n k / N}\left(v\left(k T_{s}\right)+e^{j 2 \pi n\left(k-k^{*}\right) / N}\right) \\
& +\sum_{k=k^{*}+N}^{L_{s}-1} v\left(k T_{s}\right) e^{-j 2 \pi n k / N},
\end{aligned}
$$




$$
=\sum_{k=0}^{L_{s}-1} v\left(k T_{s}\right) e^{-j 2 \pi n k / N}+\sum_{k=k^{*}}^{k^{*}+N-1} e^{-j 2 \pi n k^{*} / N}
$$

where Considering Inphase (I) and Quadrature (Q) components of $y_{n}$ and $v\left(k T_{s}\right)$ as $y_{n}^{(I)}$ and $y^{(Q)}$, and $v_{k}^{(I)}$ and $v(Q)_{k}$ respectively, we have:

$$
\begin{gathered}
y_{n}^{(I)}=N \cos \left(2 \pi n k^{*} / N\right)+\sum_{k=0}^{L_{s}-1}\left\{v_{k}^{(I)} \cos (2 \pi n k / N)+v(Q)_{k} \sin (2 \pi n k / N)\right\} \\
y_{n}^{(Q)}=-N \sin \left(2 \pi n k^{*} / N\right)+\sum_{k=0}^{L_{s}-1}\left\{v(Q)_{k} \cos (2 \pi n k / N)-v_{k}^{(I)} \sin (2 \pi n k / N)\right\}
\end{gathered}
$$

Considering A.3, A.4 and $v_{k}^{(I)}$ and $v_{k}^{(Q)} \sim N\left(0, \sigma^{2}\right)$, it is easy to show that $y_{n}^{(I)}$ and $y_{n}^{(Q)}$ also have Gaussian distributions with variance $L_{s} \sigma^{2}$, and means $\left(N \cos \left(2 \pi n k^{*} / N\right)\right)$ and $\left(-N \sin \left(2 \pi n k^{*} / N\right)\right)$, respectively. Since $z_{n}=\left|y_{n}\right|=$ $\sqrt{\left(y_{n}^{(I)}\right)^{2}+\left(y_{n}^{(I)}\right)^{2}}$, and $y_{n}^{(I)}$ and $y_{n}^{(Q)}$ are none-zero mean Gaussian random variables, $z_{n}$ would have a Rician distribution with probability density function:

$$
f_{z_{n}}\left(z_{n}\right)=\frac{z_{n}}{L_{s} \sigma^{2}} e^{-\frac{z_{n}^{2}+N^{2}}{2 L_{s} \sigma^{2}}} I_{0}\left(\frac{z_{n} N}{L_{s} \sigma^{2}}\right)
$$

where $I_{0}($.$) represents the zero order Bessel function.$ 
Using (A.5), for the probability of miss detection can be defined as:

$$
\begin{aligned}
P_{m} & =P\left(z_{n}<\lambda \mid \mathbf{s}^{(n)} \text { is within } \mathbf{r}\right) \\
& =\int_{0}^{\lambda} \frac{z_{n}}{L_{s} \sigma^{2}} e^{-\frac{z_{n}^{2}+N^{2}}{2 L_{s} \sigma^{2}}} I_{0}\left(\frac{z_{n} N}{L_{s} \sigma^{2}}\right) d z_{n} \\
& =1-Q_{1}\left(\frac{N}{\sigma \sqrt{L_{s}}}, \frac{\lambda}{\sigma \sqrt{L_{s}}}\right)
\end{aligned}
$$

where $Q_{1}$ represents the Marcum Q function and $N, L_{s}, \lambda$ and $\sigma$ are defined in 2.12. For $N_{s}$ sub-carriers in transmitted signature (here we assumed consecutive indexes such that $\mathcal{N}_{m}:=\left\{1,2, \ldots, N_{s}\right\}$ for simplicity) we would have:

$$
\begin{aligned}
P_{n} & =P\left(z_{n, 1}, \ldots, z_{n, N_{s}}<\lambda \mid \mathbf{s}^{(n, 1)}, \ldots, \mathbf{s}^{\left(n, N_{s}\right)} \text { is within } \mathbf{r}\right), \\
& =\prod_{p=1}^{N_{s}} P\left(z_{n, p}<\lambda \mid \mathbf{s}^{(n, p)} \text { is within } \mathbf{r}\right) \\
& =\left[1-Q_{1}\left(\frac{N}{\sigma \sqrt{L_{s}}}, \frac{\lambda}{\sigma \sqrt{L_{s}}}\right)\right]^{N_{s}},
\end{aligned}
$$

where $z_{n, p}=\left|\mathbf{w}_{n_{p}}^{T} \mathbf{r}\right|$ and $N, L_{s}, \lambda$ and $\sigma$ are defined in $(2.12)$. Here $n_{p}$ represents the $p^{\text {th }}$ dedicated sub-carrier to the $n^{\text {th }}$ sensor node. When the received signal does not contain the $n^{\text {th }}$ sensor node's signature, for the $n^{\text {th }}$ entry of $\mathbf{y}$ we have:

$$
y_{n}=\mathbf{w}_{n}^{T} \mathbf{r}=\sum_{k=0}^{L_{s}-1} v\left(k T_{s}\right) e^{-j 2 \pi n k / N}
$$

Considering Inphase (I) and Quadrature (Q) components of $y_{n}$ and $v\left(k T_{s}\right)$ as $y_{n}^{(I)}$ 
and $y_{n}^{(Q)}$, and $v_{k}^{(I)}$ and $v_{k}^{(Q)}$ we have:

$$
\begin{aligned}
& y_{n}^{(I)}=\sum_{k=0}^{L_{s}-1}\left\{v_{k}^{(I)} \cos (2 \pi n k / N)+v_{k}^{(Q)} \sin (2 \pi n k / N)\right\} \\
& y_{n}^{(Q)}=\sum_{k=0}^{L_{s}-1}\left\{v_{k}^{(Q)} \cos (2 \pi n k / N)-v_{k}^{(I)} \sin (2 \pi n k / N)\right\},
\end{aligned}
$$

which leads to the Rayleigh distribution for $z_{n}=\left|y_{n}\right|=\sqrt{\left(y_{n}^{(I)}\right)^{2}+\left(y_{n}^{(I)}\right)^{2}}$ with probability density function:

$$
f_{z_{n}}\left(z_{n}\right)=\frac{z_{n}}{L_{s} \sigma^{2}} e^{-\left(z_{n}^{2} / 2 L_{s} \sigma^{2}\right)}
$$

Therefore, the probability of false alarm can be defined as:

$$
P_{f}=P\left(z_{n}>\lambda \mid \mathbf{s}^{(n)} \text { is not in } \mathbf{r}\right)=e^{-\left(\lambda^{2} / 2 L_{s} \sigma^{2}\right)},
$$

where $L_{s}, \lambda$ and $\sigma$ are defined in $(2.12$.

In the case of multi-path channels for the received signal, we would have:

$$
\begin{aligned}
& y_{n}=\mathbf{w}_{n}^{T} \mathbf{r}=\sum_{k=0}^{k^{*}-1} v\left(k T_{s}\right) e^{-j 2 \pi n k / N}+ \\
& \sum_{k=k^{*}}^{k^{*}+N-1} e^{-j 2 \pi n k / N}\left(v\left(k T_{s}\right)+\sum_{l=0}^{L-1} h_{l} e^{j 2 \pi n\left(k-l-k^{*}\right) / N}\right) \\
& +\sum_{k=k^{*}+N}^{L_{s}-1} v\left(k T_{s}\right) e^{-j 2 \pi n k / N},
\end{aligned}
$$




$$
\begin{aligned}
& =\sum_{k=0}^{L_{s}-1} v\left(k T_{s}\right) e^{-j 2 \pi n k / N}+\sum_{k=k^{*}}^{k^{*}+N-1} \sum_{l=0}^{L-1} h_{l} e^{-j 2 \pi \frac{n\left(l+k^{*}\right)}{N}}, \\
& =\sum_{k=0}^{L_{s}-1} v\left(k T_{s}\right) e^{-j 2 \pi n k / N}+N \sum_{l=0}^{L-1} h_{l} e^{-j 2 \pi n\left(l+k^{*}\right) / N},
\end{aligned}
$$

where $h_{l}=h_{l}^{(I)}+j h_{l}^{(Q)}$ and $L$ denote the complex gain of $l^{\text {th }}$ channel tap and number of channel taps, respectively. Defining $\alpha_{n}=\sum_{l=0}^{L-1} h_{l} e^{-j 2 \pi n\left(l+k^{*}\right) / N}$, it is easy to show that $\alpha_{n}$ is complex Gaussian random variable where $\alpha_{n}^{(I)}$ and $\alpha_{n}^{(Q)}$ have Gaussian distribution with zero mean and variance $L \sigma_{h}^{2}$, where $\sigma_{h}^{2}$ is the variance of $h_{l}^{(I)}$ and $h_{l}^{(Q)}$. Then $\sum_{k=k^{*}}^{k^{*}+N-1} \alpha_{n}$ will have a complex Gaussian distribution with zero mean and variance $L N^{2} \sigma_{h}^{2}$. Therefore, same as A.1 and $\mathrm{A} .2$, $y_{n}$ is complex Gaussian random variable where $y_{n}^{(I)}$ and $y_{n}^{(Q)}$ have Gaussian distribution with zero mean and variance $\left(L_{s} \sigma^{2}+L N^{2} \sigma_{h}^{2}\right)$. Defining $z_{n}=\left|y_{n}\right|=\sqrt{\left(y_{n}^{(I)}\right)^{2}+\left(y_{n}^{(I)}\right)^{2}}$ and $\sigma_{z}^{2}=\left(L_{s} \sigma^{2}+L N^{2} \sigma_{h}^{2}\right), z_{n}$ would have Rayleigh distribution such that:

$$
f_{z_{n}}\left(z_{n}\right)=\frac{z_{n}}{\sigma_{z}^{2}} e^{-\left(z_{n}^{2} / 2 \sigma_{z}^{2}\right)}
$$

and finally for probability of miss detection, we would have:

$$
P_{m}=f_{z}\left(z<\lambda \mid s^{(n)}(t) \text { is in } \mathbf{r}\right)=\int_{0}^{\lambda} \frac{z}{\sigma_{z}^{2}} e^{-\left(z^{2} / 2 \sigma_{z}^{2}\right)} d z=1-e^{-\frac{\lambda^{2}}{2 \sigma_{z}^{2}}}
$$

In the case of multi-path fading and considering the received signal $\mathbf{r}$ contains the 
$n^{\text {th }}$ sensor node signature, the probability of false alarm $\left(P_{f}\right)$ is defined as:

$$
P_{f}=P\left(z_{n}>\lambda \mid \mathbf{s}^{(m)} \text { is not in } \mathbf{r}\right)
$$

where $z_{m}=\left|y_{m}\right|$ for $m=1,2, \ldots, N$ and $m \neq n$ and:

$$
y_{m}=\mathbf{w}_{m}^{T} \mathbf{r}=\sum_{k=0}^{L_{s}-1} v\left(k T_{s}\right) e^{-j 2 \pi m k / N}+\sum_{k=k^{*}}^{k^{*}+N-1} \sum_{l=0}^{L-1} h_{l} e^{-j 2 \pi\left(k(m-n)+l+k^{*}\right) / N},
$$

however, the orthogonality across sub-carriers results in:

$$
\sum_{k=k^{*}}^{k^{*}+N-1} \sum_{l=0}^{L-1} h_{l} e^{-j 2 \pi\left(k(m-n)+l+k^{*}\right) / N}=0 \text { for } m \neq n
$$

which leads to the same result as proposed in A.8). Following the same procedure of A.8)-A.11) the probability of false alarm given one transmitted signature in multi-path fading channels would lead to the same result as the one proposed in A.12.

\section{A.2 Probability of Error for ToA Estimation}

The probability of error in ToA estimation $\left(P_{e}=P\left(\hat{k}^{*} \neq k^{*}\right)\right)$ could be summarized as $P_{e}=1-P_{c}$, where $P_{c}$ represents the probability of correct ToA estimation. For 
$P_{c}$ we can write:

$$
P_{c}=P\left[c_{k^{*}}>c_{1}, c_{2}, \ldots, c_{k^{*}-1}, c_{k^{*}+1}, \ldots, c_{L_{s}-N}\right]
$$

where $c_{k}=\left|\mathbf{s}^{\mathbf{H}} \mathbf{r}_{k: k+N-1}\right|$, however $c_{k}$ has Rayleigh distribution for $1 \leq k \leq k^{*}-N$ and $k^{*}+N \leq k \leq L_{s}-N$ with parameter $N \sigma^{2}$ and Rician distribution for $k^{*}-$ $N+1 \leq k \leq k^{*}+N-1$ with $N \sigma^{2}$ and mean $m_{k}=k-\left(k^{*}-N\right)$. Therefore, for $P_{c}$ would be:

$$
P_{c}=\int_{0}^{\infty} P\left[c_{k^{*}}>c_{1}, \ldots, c_{k^{*}-1}, c_{k^{*}+1}, \ldots, c_{L_{s}-N} \mid c_{k^{*}}\right] p_{c_{k^{*}}}\left(c_{k^{*}}\right) d c_{k^{*}}
$$

and,

$$
P_{c}=\int_{0}^{\infty}\left(P\left[c_{k^{*}}>c_{1}\right]\right)^{\left(L_{s}-3 N+1\right)}\left(\prod_{k=1}^{N-1} P\left[c_{k^{*}}>c_{k^{*}-N+k}\right]\right)^{2} p_{c_{k^{*}}}\left(c_{k^{*}}\right) d c_{k^{*}}
$$

But,

$$
P\left[c_{k^{*}}>c_{1}\right]=\int_{0}^{c_{k^{*}}} \frac{c_{1}}{N \sigma^{2}} e^{-\frac{c_{1}^{2}}{2 N \sigma^{2}}} d c_{1}=1-e^{-\frac{c_{k^{*}}^{2}}{2 N \sigma^{2}}}
$$

However $c_{k^{*}}$ and $c_{k^{*}-N+k}$ are correlated since they have $k$ common noise samples and direct calculation of $P\left[c_{k^{*}}>c_{k^{*}-N+k}\right]$ would be too cumbersome. Ignoring the $k$ common noise samples within the $c_{k^{*}-N+k}$ we can consider $c_{k^{*}}$ and $c_{k^{*}-N+k}$ as two 
independent random variables which leads to:

$P\left[c_{k^{*}}>c_{k^{*}-N+k}\right]=1-P\left[c_{k^{*}-N+k}>c_{k^{*}}\right] \leq 1-Q_{1}\left(\frac{k}{\sqrt{(N-k) \sigma^{2}}}, \frac{c_{k^{*}}}{\sqrt{(N-k) \sigma^{2}}}\right)$,

Therefore, the probability of correct ToA estimation can be expressed as:

$$
\begin{array}{r}
P_{c} \leq \int_{0}^{\infty} \prod_{k=1}^{N-1}\left(1-Q_{1}\left(\frac{k}{\sqrt{(N-k) \sigma^{2}}}, \frac{c_{k^{*}}}{\sqrt{(N-k) \sigma^{2}}}\right)\right)^{2} \\
\left(1-e^{-\frac{c_{k^{*}}^{2}}{2 N \sigma^{2}}}\right)^{\left(L_{s}-3 N+1\right)} f_{c_{k^{*}}}\left(c_{k^{*}}\right) d c_{k^{*}},
\end{array}
$$

for:

$$
f_{c_{k^{*}}}\left(c_{k^{*}}\right)=\frac{c_{k^{*}}}{N \sigma^{2}} I_{0}\left(\frac{N c_{k^{*}}}{2 N \sigma^{2}}\right) e^{-\left(\frac{c_{k^{*}+N^{2}}^{2}}{2 N \sigma^{2}}\right)},
$$

where $I_{0}($.$) represents the zero order Bessel function. Finally, the probability of error$ could be represented $P_{e}=1-P_{c}$. For $N_{s}$ sub-carriers (assuming $\mathcal{N}_{m}:=\left\{1,2, \ldots, N_{s}\right\}$ for simplicity) in transmitted signature, $P_{e}$ is obtained following (A.28)-A.34).

$$
P_{c}=P\left[\prod_{p=1}^{N_{s}} c_{k^{*}}^{(p)}>\prod_{p=1}^{N_{s}} c_{1}^{(p)}, \prod_{p=1}^{N_{s}} c_{2}^{(p)}, \ldots, \prod_{p=1}^{N_{s}} c_{k^{*}-1}^{(p)}, \prod_{p=1}^{N_{s}} c_{k^{*}+1}^{(p)}, \ldots, \prod_{p=1}^{N_{s}} c_{L_{s}-N}^{(p)}\right]
$$

where $c_{k}^{(p)}=\left|\left(\mathbf{s}^{(p)}\right)^{H} \mathbf{r}_{k: k+N-1}\right|$. This leads to:

$$
\begin{gathered}
P_{c}=\int_{0}^{\infty} \ldots \int_{0}^{\infty} P\left[\prod_{p=1}^{N_{s}} c_{k^{*}}^{(p)}>\prod_{p=1}^{N_{s}} c_{1}^{(p)}, \ldots, \prod_{p=1}^{N_{s}} c_{k^{*}-1}^{(p)}, \prod_{p=1}^{N_{s}} c_{k^{*}+1}^{(p)}, \ldots\right. \\
\left.\prod_{p=1}^{N_{s}} c_{L_{s}-N}^{(p)} \mid c_{k^{*}}^{(1)}, \ldots, c_{k^{*}}^{\left(N_{s}\right)}\right] f_{c_{k^{*}}}^{(1)}\left(c_{k^{*}}^{(1)}\right) \ldots f_{c_{k^{*}}^{\left(N_{s}\right)}}\left(c_{k^{*}}^{\left(N_{s}\right)}\right) d c_{k^{*}}^{(1)} \ldots d c_{k^{*}}^{\left(N_{s}\right)}
\end{gathered}
$$




$$
\begin{gathered}
P_{c}=\int_{0}^{\infty} \ldots \int_{0}^{\infty}\left(P\left[\prod_{p=1}^{N_{s}} c_{k^{*}}^{(p)}>\prod_{p=1}^{N_{s}} c_{1}^{(p)}\right]\right)^{\left(L_{s}-3 N+1\right)} \\
\left(\prod_{k=1}^{N-1} P\left[\prod_{p=1}^{N_{s}} c_{k^{*}}^{(p)}>\prod_{p=1}^{N_{s}} c_{k^{*}-N+k}^{(p)}\right]\right)^{2} \\
f_{c_{k^{*}}^{(1)}}\left(c_{k^{*}}^{(1)}\right) \ldots f_{c_{k^{*}}^{\left(N_{s}\right)}}\left(c_{k^{*}}^{\left(N_{s}\right)}\right) d c_{k^{*}}^{(1)} \ldots d c_{k^{*}}^{\left(N_{s}\right)}
\end{gathered}
$$

where $c_{k}^{(p)}$ for $1 \leq p \leq N_{s}$, has Rayleigh distribution and $1 \leq k \leq k^{*}-N$ and $k^{*}+N \leq k \leq L_{s}-N$ with parameter $N \sigma^{2}$ and Rician distribution for $k^{*}-N+1 \leq$ $k \leq k^{*}+N-1$ with $N \sigma^{2}$ and mean $m_{k}=k-\left(k^{*}-N\right)$ however;

$$
\begin{gathered}
P\left[\prod_{p=1}^{N_{s}} c_{k^{*}}^{(p)}>\prod_{p=1}^{N_{s}} c_{1}^{(p)}\right]=\int_{0}^{\infty} \ldots \int_{0}^{\infty} P\left[\prod_{p=1}^{N_{s}} c_{k^{*}}^{(p)}>\prod_{p=1}^{N_{s}} c_{1}^{(p)} \mid c_{1}^{(2)}, \ldots, c_{1}^{\left(N_{s}\right)}\right] \\
f_{c_{1}^{(2)}}\left(c_{1}^{(2)}\right) \ldots f_{c_{1}^{\left(N_{s}\right)}}\left(c_{1}^{\left(N_{s}\right)}\right) d c_{1}^{(2)} \ldots d c_{1}^{\left(N_{s}\right)}, \\
P\left[\prod_{p=1}^{N_{s}} c_{k^{*}}^{(p)}>\prod_{p=1}^{N_{s}} c_{k^{*}-N+k}^{(p)}\right]=\int_{0}^{\infty} \ldots \int_{0}^{\infty} P\left[\prod_{p=1}^{N_{s}} c_{k^{*}}^{(p)}>\prod_{p=1}^{N_{s}} c_{1}^{(p)} \mid c_{k^{*}-N+k}^{(2)}, \ldots, c_{k^{*}-N+k}^{\left(N_{s}\right)}\right] \\
f_{c_{k^{*}-N+k}^{(2)}}\left(c_{k^{*}-N+k}^{(2)}\right) \ldots f_{c_{k^{*}-N+k}^{\left(N_{s}\right)}}\left(c_{k^{*}-N+k}^{\left(N_{s}\right)}\right) d c_{k^{*}-N+k}^{(2)} \ldots d c_{k^{*}-N+k}^{\left(N_{s}\right)},
\end{gathered}
$$

To finalize the proposed integral equations, we use the same approach as (53) and (54) to introduce:

$$
P\left[\prod_{p=1}^{N_{s}} c_{k^{*}}^{(p)}>\prod_{p=1}^{N_{s}} c_{1}^{(p)} \mid c_{1}^{(2)}, \ldots, c_{1}^{\left(N_{s}\right)}\right]=\int_{0}^{\beta} \frac{c_{1}^{(1)}}{N \sigma^{2}} e^{-\frac{\left(c_{1}^{(1)}\right)^{2}}{2 N \sigma^{2}}} d c_{1}^{(1)}=1-e^{-\frac{\beta^{2}}{2 N \sigma^{2}}}
$$




$$
\begin{aligned}
P\left[\prod_{p=1}^{N_{s}} c_{k^{*}}^{(p)}>\right. & \left.\prod_{p=1}^{N_{s}} c_{k^{*}-N+k}^{(p)} \mid c_{k^{*}-N+k}^{(2)}, \ldots, c_{k^{*}-N+k}^{\left(N_{s}\right)}\right] \\
& \leq 1-Q_{1}\left(\frac{k}{\sqrt{(N-k) \sigma^{2}}}, \frac{\gamma}{\sqrt{(N-k) \sigma^{2}}}\right)
\end{aligned}
$$

for $\beta=\frac{\prod_{p=1}^{N_{s}} c_{k^{*}}^{(p)}}{\prod_{p=2}^{N_{s}} c_{1}^{(p)}}$ and $\gamma=\frac{\prod_{p=1}^{N_{s}} c_{k^{*}}^{(p)}}{\prod_{p=2}^{N_{s}} c_{k^{*}-N+k}^{(p)}}$ and $c_{k^{*}}^{(p)}=\left|\left(\mathbf{s}^{(p)}\right)^{H} \mathbf{r}_{k^{*}: k^{*}+N-1}\right|$.

\section{A.3 First Order Derivative of WISL Objective}

\section{Function}

Considering (3.15), we define:

$$
W I S L=\frac{1}{4 N} \sum_{p=1}^{2 N}\left(S_{p}-\Gamma_{1,1} N\right)^{2},
$$

for $S_{p}=\tilde{\mathbf{s}_{\mathbf{p}}^{\dagger}} \tilde{\Gamma \tilde{\mathbf{p}}}$, where,

$$
\tilde{\mathbf{s}}_{p}=\left[e^{j \varphi_{1}} e^{-j \frac{\pi p}{N}}, \ldots, e^{j \varphi_{N}} e^{-j \frac{\pi N p}{N}}\right]^{T}
$$

where $\left\{\varphi_{n}\right\}_{n=1}^{N}$ is the desired phase sequence of waveform with length $N$ defined in (3.14), and $\Gamma$ is defined in 3.17). 
Substituting A.36 into A.35 leads to:

$$
\begin{array}{r}
S_{p}=\sum_{l=1}^{N} \sum_{k=1}^{N} e^{-j \varphi_{k}} e^{j \frac{\pi k p}{N}} \Gamma_{k, l} e^{j \varphi_{l}} e^{-j \frac{\pi l p}{N}} \\
=\sum_{l=1}^{N} e^{j \varphi_{l}} e^{-j \frac{\pi l p}{N}} \sum_{k=1, k \neq l}^{N} e^{-j \varphi_{k}} e^{j \frac{\pi k p}{N}} \Gamma_{k, l}+\sum_{l=1}^{N} \Gamma_{l, l}
\end{array}
$$

The real part of A.37 can be represented by:

$$
\begin{aligned}
R e\left\{S_{p}\right\} & =\operatorname{Re}\left\{\sum_{l=1}^{N} e^{j \varphi_{l}} e^{-j \frac{\pi l p}{N}} \sum_{k=1, k \neq l}^{N} e^{-j \varphi_{k}} e^{j \frac{\pi k p}{N}} \Gamma_{k, l}+\sum_{l=1}^{N} \Gamma_{l, l}\right\} \\
& =\sum_{l=1}^{N}\left[\cos \alpha_{l} \sum_{k=1, k \neq l}^{N} \cos \alpha_{k} \Gamma_{k, l}+\sin \alpha_{l} \sum_{k=1, k \neq l}^{N} \sin \alpha_{k} \Gamma_{k, l}\right]
\end{aligned}
$$

where $\alpha_{l}=\varphi_{l}-\frac{\pi l p}{N}$. Substituting A.38 into:

$$
\frac{\partial W I S L}{\partial \varphi_{m}}=\frac{\partial}{\partial \varphi_{m}}\left\{|S p|^{2}-2 N R e\left\{S_{p} \Gamma_{1,1}\right\}\right\}
$$

leads to:

$$
\begin{array}{r}
\frac{\partial W I S L}{\partial \varphi_{m}}=2 S_{p} \frac{\partial}{\partial \varphi_{m}}\left(\sum_{l=1}^{N} \sum_{k=1}^{N} e^{-j \varphi_{k}} e^{j \frac{\pi k p}{N}} \Gamma_{k, l} e^{j \varphi_{l}} e^{-j \frac{\pi l p}{N}}\right)^{*} \\
-2 N \frac{\partial}{\partial \varphi_{m}}\left\{\sum_{l=1}^{N}\left[\cos \alpha_{l} \sum_{k=1, k \neq l}^{N} \cos \alpha_{k} \Gamma_{k, l}+\sin \alpha_{l} \sum_{k=1, k \neq l}^{N} \sin \alpha_{k} \Gamma_{k, l}\right]\right\}
\end{array}
$$


the first term in A.40 can be mathematically manipulated into:

$$
\begin{aligned}
& \frac{\partial}{\partial \varphi_{m}}\left(\sum_{l=1}^{N} \sum_{k=1}^{N} e^{-j \varphi_{k}} e^{j \frac{\pi k p}{N}} \Gamma_{k, l} e^{j \varphi_{l}} e^{-j \frac{\pi l p}{N}}\right)^{*} \\
& =-j e^{-j \varphi_{m}} e^{j \frac{\pi m p}{N}} \sum_{k=1, k \neq m}^{N} e^{j \varphi_{k}} e^{-j \frac{\pi k p}{N}} \Gamma_{k, m}+j e^{j \varphi_{m}} e^{-j \frac{\pi m p}{N}} \\
& \sum_{k=1, k \neq m}^{N} e^{-j \varphi_{k}} e^{j \frac{\pi k p}{N}} \Gamma_{m, k}=-2 \sum_{k=1, k \neq m}^{N} \sin \left(\alpha_{k}-\alpha_{m}\right) \Gamma_{m, k}
\end{aligned}
$$

Applying the same procedure, the second term in A.40 can be written by:

$$
\begin{aligned}
& \frac{\partial}{\partial \varphi_{m}}\left\{\sum _ { l = 1 } ^ { N } \left[\cos \alpha_{l} \sum_{k=1, k \neq l}^{N} \cos \alpha_{k} \Gamma_{k, l}\right.\right. \\
& \left.\left.+\sin \alpha_{l} \sum_{k=1, k \neq l}^{N} \sin \alpha_{k} \Gamma_{k, l}\right]\right\}=-\sin \alpha_{m} \sum_{k=1, k \neq m}^{N} \cos \alpha_{k} \Gamma_{k, m} \\
& +\cos \alpha_{m} \sum_{k=1, k \neq m}^{N} \sin \alpha_{k} \Gamma_{k, m}-\sin \alpha_{m} \sum_{l=1, l \neq m}^{N} \cos \alpha_{l} \Gamma_{m, l} \\
& +\cos \alpha_{m} \sum_{l=1, l \neq m}^{N} \sin \alpha_{l} \Gamma_{m, l}=2 \sum_{k=1, k \neq m}^{N} \sin \left(\alpha_{k}-\alpha_{m}\right) \Gamma_{k, m}
\end{aligned}
$$

substituting $\sqrt{\mathrm{A} .41}$ ) and $(\mathrm{A} .42)$ into $(\mathrm{A} .40)$, leads to the final equation for the first order derivative of WISL as follow:

$$
\frac{\partial C p}{\partial \varphi_{m}}=\left(\frac{S_{p}}{N}-\Gamma_{1,1}\right) \sum_{k=1, k \neq m}^{N} \sin \left(\alpha_{k}-\alpha_{m}\right) \Gamma_{k, m}
$$




\section{A.4 Second Order Derivative of WISL Objective}

\section{Function}

The Hessian matrix corresponding the WISL can be represented by:

$$
\begin{aligned}
& H_{l, m}=\frac{\partial}{\partial \varphi_{l}}\left\{\frac{\partial W I S L}{\partial \varphi_{m}}\right\}= \\
& \frac{\partial}{\partial \varphi_{l}}\left\{\sum_{p=1}^{2 N}\left(\frac{S_{p}}{N}-\Gamma_{1,1}\right) \sum_{k=1, k \neq m}^{N} \sin \left(\alpha_{k}-\alpha_{m}\right) \Gamma_{k, m}\right\} \\
& =\sum_{p=1}^{2 N}\left(\frac{S_{p}}{N}-\Gamma_{1,1}\right) \frac{\partial}{\partial \varphi_{l}} \sum_{k=1, k \neq m}^{N} \sin \left(\alpha_{k}-\alpha_{m}\right) \Gamma_{k, m} \\
& +\sum_{k=1, k \neq m}^{N} \sin \left(\alpha_{k}-\alpha_{m}\right) \Gamma_{k, m} \frac{\partial}{\partial \varphi_{l}} \sum_{p=1}^{2 N}\left(\frac{S_{p}}{N}-\Gamma_{1,1}\right)
\end{aligned}
$$

where $\alpha_{l}=\varphi_{l}-\frac{\pi l p}{N}$ for $\left\{\varphi_{n}\right\}_{n=1}^{N}$ as the desired phase sequence of waveform with length $N$ defined in (3.14), and $\Gamma$ is defined in 3.17). The first term in A.44) can be mathematically manipulated into:

$$
\frac{\partial}{\partial \varphi_{l}} \sum_{k=1, k \neq m}^{N} \sin \left(\alpha_{k}-\alpha_{m}\right) \Gamma_{k, m}=\left\{\begin{array}{cc}
\cos \left(\alpha_{k}-\alpha_{m}\right) \Gamma_{m, l}, & m \neq l \\
-\sum_{k=1, k \neq m}^{N} \cos \left(\alpha_{k}-\alpha_{m}\right) \Gamma_{m, k}, m=l
\end{array}\right.
$$

Applying the same procedure, the second term in A.44 can be written by:

$$
\frac{\partial}{\partial \varphi_{l}} \sum_{p=1}^{2 N}\left(\frac{S_{p}}{N}-\Gamma_{1,1}\right)=\frac{1}{N} \sum_{p=1}^{2 N} \frac{\partial}{\partial \varphi_{l}} S_{p}=\frac{2}{N} \sum_{p=1}^{2 N} \sum_{n=1}^{N} \sin \left(\alpha_{n}-\alpha_{m}\right) \Gamma_{n, l}
$$


Therefore, the final equation for the Hessian matrix of WISL is achievable by substituting A.45 and A.46 into A.44:

$$
\begin{aligned}
& \frac{\partial}{\partial \varphi_{l}}\left\{\frac{\partial W I S L}{\partial \varphi_{m}}\right\}= \\
& \frac{2}{N} \sum_{p=1}^{2 N} \sum_{n=1}^{N} \sin \left(\alpha_{n}-\alpha_{m}\right) \Gamma_{n, l} \sum_{k=1, k \neq m}^{N} \sin \left(\alpha_{k}-\alpha_{m}\right) \Gamma_{m, k}+ \\
& \left\{\begin{array}{cc}
\sum_{p=1}^{2 N}\left(\frac{S_{p}}{N}-\Gamma_{1,1}\right) \cos \left(\alpha_{k}-\alpha_{m}\right) \Gamma_{m, l}, & m \neq l \\
-\sum_{p=1}^{2 N}\left(\frac{S_{p}}{N}-\Gamma_{1,1}\right) \sum_{k=1, k \neq m}^{N} \cos \left(\alpha_{k}-\alpha_{m}\right) \Gamma_{m, k}, m=l
\end{array}\right.
\end{aligned}
$$

\section{A.5 Details of ToA CRLB Calculation}

The derivatives of log-likelihood function with respect to the elements of unknown vector, $\mathbf{x}$ can be represented by [140]:

$$
\nabla_{\mathbf{d}}=\frac{2}{\sigma^{2}} \operatorname{Re}\left\{\operatorname{diag}\{\mathbf{h}\}\left(A^{\prime}\right)^{H}(\mathbf{r}-A \mathbf{h})\right\}
$$

where $A^{\prime}$ is defined by:

$$
A^{\prime}=\left[\begin{array}{ccc}
\frac{\partial s\left(T_{s}-\tau_{0}\right)}{\partial \tau_{0}} & \ldots & \frac{\partial s\left(T_{s}-\tau_{L-1}\right)}{\partial \tau_{L-1}} \\
\vdots & \ddots & \vdots \\
\frac{\partial s\left(N T_{s}-\tau_{0}\right)}{\partial \tau_{0}} & \cdots & \frac{\partial s\left(N T_{s}-\tau_{L-1}\right)}{\partial \tau_{L-1}}
\end{array}\right]
$$


where,

$$
\frac{\partial s\left(n T_{s}-\tau_{l}\right)}{\partial \tau_{l}}=\frac{\partial}{\partial \tau_{l}} \int S(\omega) e^{j \omega\left(t-\tau_{l}\right)} d \omega
$$

where $S(\omega)$ represents the Fourier transform of $s(t)$. Furthermore

$$
\begin{gathered}
\nabla_{\mathbf{h}^{(I)}}=\frac{2}{\sigma^{2}} \operatorname{Re}\left\{A^{H}(\mathbf{r}-A \mathbf{h})\right\}, \\
\nabla_{\mathbf{h}^{(Q)}}=\frac{2}{\sigma^{2}} \operatorname{Im}\left\{A^{H}(\mathbf{r}-A \mathbf{h})\right\},
\end{gathered}
$$

Applying the expectation into (A.48), A.51) and (A.52) leads to:

$$
\begin{gathered}
E\left\{\nabla_{\mathbf{d}} \Lambda_{\mathbf{r} \mid \mathbf{x}} \cdot \nabla_{\mathbf{d}}^{T} \Lambda_{\mathbf{r} \mid \mathbf{x}}\right\}=\frac{2}{\sigma^{2}} \operatorname{Re}\left\{\operatorname{diag}\left(\mathbf{h}^{*}\right) A^{\prime H} A^{\prime} \operatorname{diag}(\mathbf{h})\right\}, \\
E\left\{\nabla_{\mathbf{d}} \Lambda_{\mathbf{r} \mid \mathbf{x}} \cdot \nabla_{\mathbf{h}^{(I)}}^{T} \Lambda_{\mathbf{r} \mid \mathbf{x}}\right\}=\frac{2}{\sigma^{2}} \operatorname{Re}\left\{\operatorname{diag}\left(\mathbf{h}^{*}\right) A^{\prime H} A\right\}, \\
E\left\{\nabla_{\mathbf{d}} \Lambda_{\mathbf{r} \mid \mathbf{x}} \cdot \nabla_{\mathbf{h}^{(Q)}}^{T} \Lambda_{\mathbf{r} \mid \mathbf{x}}\right\}=\frac{-2}{\sigma^{2}} \operatorname{Im}\left\{\operatorname{diag}\left(\mathbf{h}^{*}\right) A^{\prime H} A\right\}, \\
E\left\{\nabla_{\mathbf{h}^{(I)}} \Lambda_{\mathbf{r} \mid \mathbf{x}} \cdot \nabla_{\mathbf{h}^{(I)}}^{T} \Lambda_{\mathbf{r} \mid \mathbf{x}}\right\}=E\left\{\nabla_{\mathbf{h}^{(Q)}} \Lambda_{\mathbf{r} \mid \mathbf{x}} \cdot \nabla_{\mathbf{h}^{(Q)}}^{T} \Lambda_{\mathbf{r} \mid \mathbf{x}}\right\}=\frac{2}{\sigma^{2}} \operatorname{Re}\left\{A^{H} A\right\}, \\
E\left\{\nabla_{\mathbf{h}^{(I)}} \Lambda_{\mathbf{r} \mid \mathbf{x}} \cdot \nabla_{\mathbf{h}^{(Q)}}^{T} \Lambda_{\mathbf{r} \mid \mathbf{x}}\right\}=E\left\{\nabla_{\mathbf{h}^{(Q)}} \Lambda_{\mathbf{r} \mid \mathbf{x}} \cdot \nabla_{\mathbf{h}^{(I)}}^{T} \Lambda_{\mathbf{r} \mid \mathbf{x}}\right\}=\frac{-2}{\sigma^{2}} \operatorname{Im}\left\{A^{H} A\right\},
\end{gathered}
$$




\section{A.6 Derivation of the Maximum Likelihood Esti-}

\section{mator}

Substituting $2(\alpha+\beta)$ with $n$ in 4.21$), K$ independent measurements can be written as:

$$
X_{k}=z_{0} e^{-j \frac{2 \pi m_{k}}{N} n}+\tilde{V}_{k}, \forall k=1,2, \ldots, K
$$

where $V_{k}$ denote the frequency domain noise. Assuming zero mean Gaussian distribution for $\tilde{V}_{k}$, the likelihood function of measurements can be written as:

$$
f\left(X_{1}, \ldots, X_{K} \mid z_{0}, n\right)=\frac{1}{\left(2 \pi \sigma_{V}^{2}\right)^{K / 2}} \prod_{k=1}^{K} e^{-\frac{\left|X_{k}-z_{0} e^{-j \frac{2 \pi m_{k}}{N} n}\right|^{2}}{2 \sigma_{V}^{2}}}
$$

where, $\sigma_{V}^{2}$ is the variance of frequency domain noise $\tilde{V}_{k}, \forall k=1,2, \ldots, K$ and $m_{k}, N$, and $K$, and $z_{0}$ are defined in 4.10 and 4.21, respectively.

The maximum likelihood estimator of $n$, can be represented by maximizing the proposed log-likelihood function w.r.t $n$ such that:

$$
\hat{n}=\underset{z_{0}, n}{\operatorname{argmax}}\left\{f\left(X_{1}, \ldots, X_{K} \mid z_{0}, n\right)\right\}
$$


for,

$$
\begin{aligned}
& =\underset{z_{0}, n}{\operatorname{argmax}}\left\{\ln \frac{1}{\left(2 \pi \sigma_{V}^{2}\right)^{K / 2}}\right. \\
& \left.-\frac{1}{2 \sigma_{V}^{2}} \sum_{k=1}^{K}\left|X_{k}-z_{0} e^{-j \frac{2 \pi m_{k}}{N} n}\right|^{2}\right\}, \\
& =\underset{z_{0}, n}{\operatorname{argmin}}\left\{\sum_{k=1}^{K}\left|X_{k}-z_{0} e^{-j \frac{2 \pi m_{k}}{N} n}\right|^{2}\right\}, \\
& =\underset{z_{0}, n}{\operatorname{argmin}}\left\{\sum_{k=1}^{K}\left|X_{k}\right|^{2}-X_{k} z_{0}^{*} e^{j \frac{2 \pi m_{k}}{N} n}\right. \\
& \left.-X_{k}^{*} z_{0} e^{-j \frac{2 \pi m_{k}}{N} n}+z_{0} z_{0}^{*}\right\},
\end{aligned}
$$

taking derivative from A.63 and solve it w.r.t $z_{0}$, leads to:

$$
\hat{z}_{0}^{*}=X_{k}^{*} e^{-j \frac{2 \pi m_{k}}{N} n}
$$

substituting A.64 into A.63 and solving for $n$, leads to:

$$
\begin{aligned}
\hat{n}=\underset{n}{\operatorname{argmax}} & \left\{2 \sum_{k=1}^{K} X_{k} X_{k}^{*} e^{-j \frac{2 \pi m_{k}}{N} n} e^{j \frac{2 \pi m_{k}}{N} n}\right\}, \\
& =\underset{n}{\operatorname{argmax}}\left|W_{n}^{T} \mathbf{x}\right|^{2}
\end{aligned}
$$

where $\mathbf{w}_{n}=\left[e^{j \frac{2 \pi K n}{N}}, e^{j \frac{2 \pi(K-1) n}{N}}, \ldots, e^{j \frac{2 \pi n}{N}}\right]^{T}$ is $K \times 1$ column vector and $\mathbf{x}=$ $\left[X_{1}, X_{2}, \ldots, X_{K}\right]^{T}$. 


\section{A.7 Deriving Straight-Line Range}

Considering Fig. 5.1 we can write:

$$
r=\frac{D}{\cos (\theta)}
$$

where $D=\sum_{m=1} z_{m}$. Therefore, it is desired to find an expression which substitutes $\theta$ as function of known and estimated parameters as follow.

$$
\tan (\theta)=\frac{1}{D} \sum_{m=1}^{M} z_{m} \tan \left(\theta_{m}^{(q)}\right)
$$

where $M, z_{m}, \theta_{m}^{(q)}$ are defined in. Based on Snell's law, it can be shown that:

$$
\theta_{m}^{(q)}=\sin ^{-1}\left(\sin \left(\theta_{M}^{(q)}\right) \frac{\sqrt{\varepsilon_{r, m}^{(q)}}}{\sqrt{\varepsilon_{r, M}^{(q)}}}\right),
$$

Substituting A.69 into A.68 leads to:

$$
\begin{aligned}
\tan (\theta) & =\frac{1}{D} \sum_{m=1}^{M} z_{m} \tan \left(\sin ^{-1}\left(\sin \left(\theta_{M}^{(q)}\right) \frac{\sqrt{\varepsilon_{r, m}^{(q)}}}{\sqrt{\varepsilon_{r, M}^{(q)}}}\right)\right) \\
= & \frac{1}{D} \sum_{m=1}^{M} z_{m} \frac{\sin \left(\theta_{M}^{(q)}\right) \frac{\sqrt{\varepsilon_{r, m}^{(q)}}}{\sqrt{\varepsilon_{r, M}^{(q)}}}}{\cos \left(\sin ^{-1}\left(\sin \left(\theta_{M}^{(q)}\right) \frac{\sqrt{\varepsilon_{r, m}^{(q)}}}{\sqrt{\varepsilon_{r, M}^{(q)}}}\right)\right)}
\end{aligned}
$$


Using $\cos \left(\sin ^{-1}(x)\right)=\sqrt{1-x^{2}}$ and applying some mathematical manipulations it can be shown that:

$$
\tan (\theta)=\frac{1}{D} \sum_{m=1}^{M} \frac{z_{m} \sin \left(\theta_{M}^{(q)}\right) \sqrt{\varepsilon_{r, m}^{(q)}}}{\sqrt{\varepsilon_{r, M}^{(q)}-\sin ^{2}\left(\theta_{M}^{(q)}\right) \varepsilon_{r, m}^{(q)}}}
$$

Applying A.72 into A.67) and substituting $\cos \left(\tan ^{-1}(x)\right)=\frac{1}{\sqrt{1+x^{2}}}$, the straightline range between transmitter and receiver as function of measured thicknesses and DoAs can be written as:

$$
\hat{r}^{(q)}=D \sqrt{1+\left[\frac{1}{D} \sum_{m=1}^{M} \frac{z_{m} \sin \left(\theta_{M}^{(q)}\right) \sqrt{\varepsilon_{r, m}^{(q)}}}{\sqrt{\varepsilon_{r, M}^{(q)}-\sin ^{2}\left(\theta_{M}^{(q)}\right) \varepsilon_{r, m}^{(q)}}}\right]^{2}},
$$





\section{Appendix B}

\section{Publication Copyright Letter for}

Chapter 2 


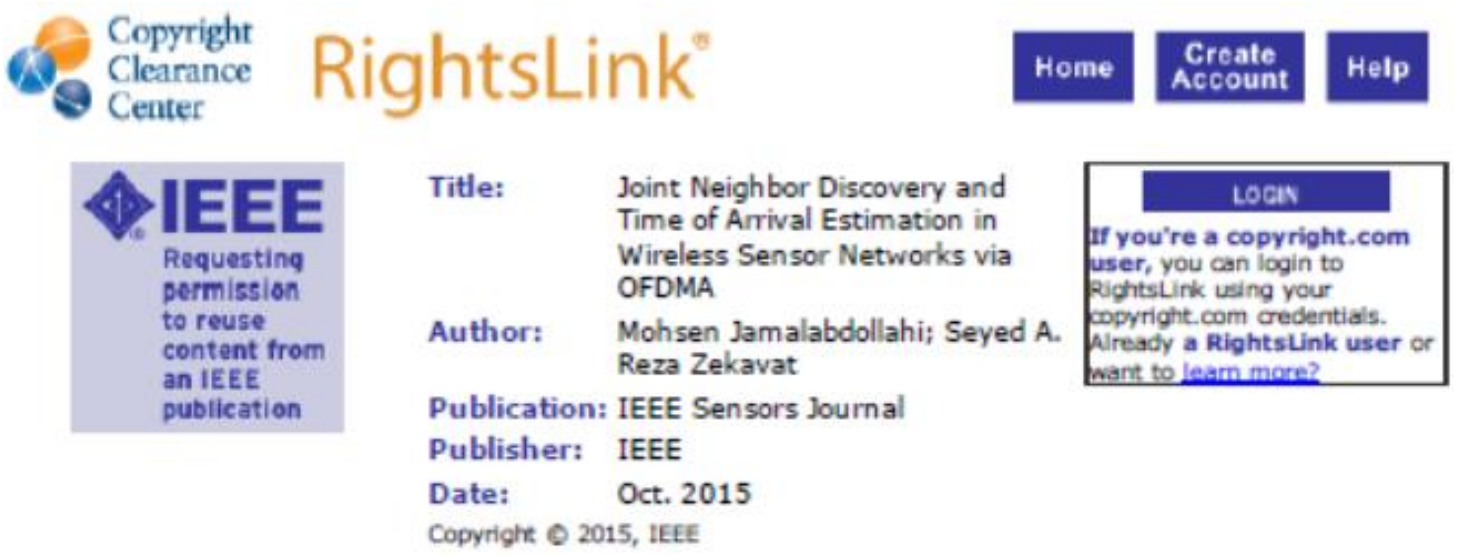

Thesis / Dissertation Reuse

The IEEE does not require individuals working on a thesis to obtain a formal reuse license, however, you may print out this statement to be used as a permission grant:

Requirements to be followed when using any portion (e.g., figure, graph, table, or textual material) of an IEEE copyrighted paper in a thesis:

1) In the case of textual material (e.g., using short quotes or referring to the work within these papers) users must give full credit to the original source (author, paper, publication) followed by the IEEE copyright line (c) 2011 IEEE.

2) In the case of illustrations or tabular material, we require that the copyright line (C) [Year of original publication] IEEE appear prominently with each reprinted figure and/or table.

3) If a substantial portion of the original paper is to be used, and if you are not the senior author, also obtain the senior author's approval.

Requirements to be followed when using an entire IEEE copyrighted paper in a thesis:

1) The following IEEE copyright/ credit notice should be placed prominently in the references: (c) [year of original publication] IEEE. Reprinted, with permission, from [author names, paper title, IEEE publication title, and month/year of publication]

2) Only the accepted version of an IEEE copyrighted paper can be used when posting the paper or your thesis on-line.

3) In placing the thesis on the author's university website, please display the following message in a prominent place on the website: In reference to IEEE copyrighted material which is used with permission in this thesis, the IEEE does not endorse any of [university/educational entity's name goes here]'s products or services. Internal or personal use of this material is permitted. If interested in reprinting/republishing IEEE copyrighted material for advertising or promotional purposes or for creating new collective works for resale or redistribution, please go to http://www.ieee.org/publications standards/publications/riqhts/rights link.html to learn how to obtain a License from RightsLink. 